\title{
DIELECTRIC LOSS DETERMINATION USING PERTURBATION
}

\author{
by \\ Madeleine Y. Andrawis \\ Dissertation submitted to the Faculty of the \\ Virginia Polytechnic Institute and State University \\ in partial fulfillment of the requirements for the degree of \\ Ph.D. \\ in
}

Electrical Engineering

APPROVED:

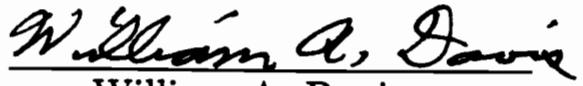

William A. Davis,

Co-Chairperson

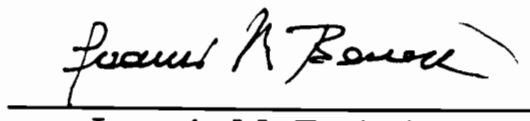

Ioannis M. Besieris
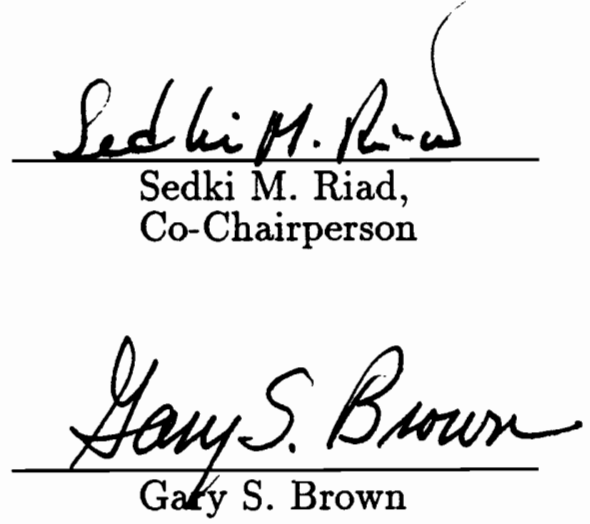

Tatemen E. Yoton

Werner E. Kohler

December, 1991

Blacksburg, Virginia 


\title{
DIELECTRIC LOSS DETERMINATION USING PERTURBATION
}

\author{
by \\ Madeleine Y. Andrawis \\ Professors William A. Davis and Sedki M. Riad, Co-Chairs
}

(ABSTRACT)

A dielectric filled cavity structure is currently being used to estimate the dielectric constant and loss factor over a wide range of frequencies of a dielectric material which fills the cavity structure [Saed, 1987]. A full field analysis is used to compute the effective complex permittivity of the sample material based on reflection coefficient measurements of the cavity structure and associated geometrical dimensions. The method has previously been used successfully to determine the dielectric constant of materials, but limitations in the method have created difficulties in accurate determination of the dielectric loss factor. The effective loss in this method yields an estimate of the total cavity loss, including both the dielectric loss and that of the cavity conductor walls.

In this dissertation a perturbation approach is used to separate the conductor loss from the total loss. The loss-free full-field analysis is used to determine the electric current at the conductor boundaries. This current is used to evaluate the perturbed power dissipated in the cavity walls based on known conductor properties. By subtracting the loss due to the conductor walls from the total loss measured in the structure, the dielectric loss and the resultant dielectric loss factor may be estimated.

Measurements are presented for sample dielectric materials. The dielectric loss tangents computed by this new technique improve the unperturbed estimates in the microwave frequency range. 


\section{ACKNOWLEDGEMENT}

I would like to express my sincere gratitude to Dr. Bill Davis for his sincere help, support, and guidance throughout this work. Without his moral support and technical assistance this dissertation could have never been completed. I would also like to thank Dr. Sedki Riad for his help and support throughout the period of this work.

Special thanks are also due to the members of my advisory committee: Dr. Besieris, Dr. Brown, Dr. Elshabini-Riad, and Dr. Kholer for their time, help and invaluable comments.

I would also like to express my deep gratitude to my kind and loving husband Alfred for his encouragement and support. Very special thanks to my son Dave and my daughter Mary for understanding, babisitting their younger brother, cleaning and even cooking during busy times. Thanks also to my youngest Virginian son Danny for cooperating and letting me sit and do my "homework" on my computer at home for long hours. Last but not least my thanks to my family for their continuous prayers and support.

Finally, I would like to thank all my colleagues in the Time Domain Lab at Virginia Tech for their kindness and frienship.

This work was supported in part by Du Pont Electronics, E. I. Du Pont de Nemours \& Co. (Inc.), Wilmington, Delaware.

Madeleine Y. Andrawis 


\section{TABLE OF CONTENTS}

ChapterTitle $\quad \underline{\text { Page }}$

1 INTRODUCTION $\ldots \ldots \ldots \ldots \ldots \ldots \ldots \ldots \ldots \ldots \ldots \ldots \ldots \ldots \ldots \ldots$

2 THE UNPERTURBED PROBLEM $\ldots \ldots \ldots \ldots \ldots \ldots \ldots \ldots \ldots$

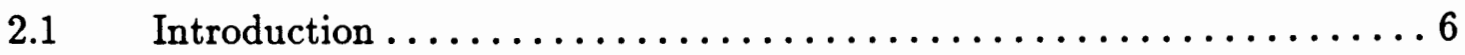

$2.2 \quad$ General green's function approaxch $\ldots \ldots \ldots \ldots \ldots \ldots \ldots \ldots$

$2.3 \quad$ Solution for the green's function $\ldots \ldots \ldots \ldots \ldots \ldots \ldots \ldots \ldots \ldots$

$2.4 \quad$ Solution to the unperturbed problem $\ldots \ldots \ldots \ldots \ldots \ldots \ldots \ldots \ldots \ldots \ldots$

2.5 Aperture fields comparison and mode determination $\ldots \ldots \ldots \ldots 25$

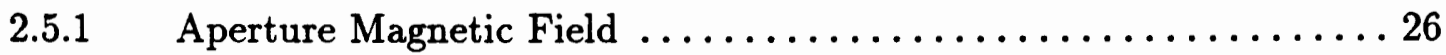

2.5.2 Aperture Electric Field ....................... 27

2.5.3 Number of Modes Determination.$\ldots \ldots \ldots \ldots \ldots \ldots \ldots \ldots \ldots$

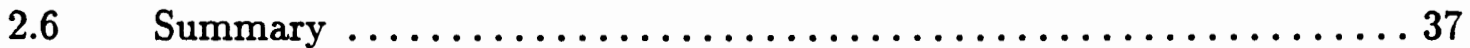

3 THE PERTURBATION PROBLEM $\ldots \ldots \ldots \ldots \ldots \ldots \ldots \ldots$

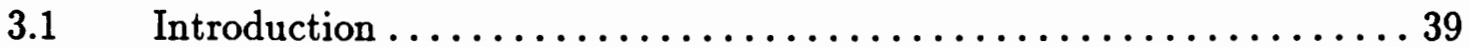

3.2 A canonical perturbation example $\ldots \ldots \ldots \ldots \ldots \ldots \ldots \ldots \ldots$

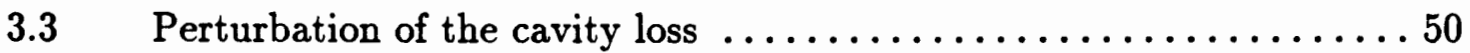

3.3.1 Real power absorbed by the unperturbed cavity ........... 57

3.3.2 Real power dissipated in the walls of the perturbed cavity ......58

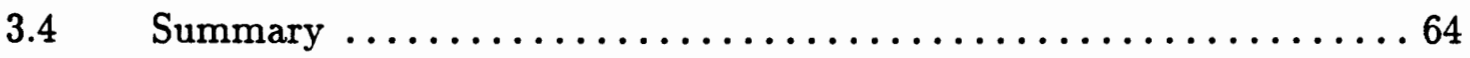

4 SIMULATION AND EXPERIMENTAL RESULTS $\ldots \ldots \ldots \ldots \ldots 6$

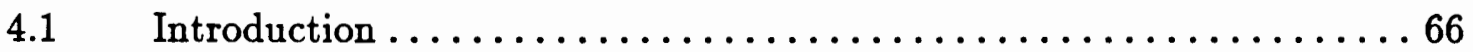

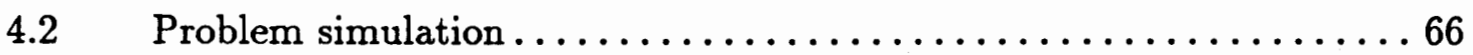

4.3 Dielectric loss determination of pyralux ${ }^{\circledR} \ldots \ldots \ldots \ldots \ldots \ldots \ldots 72$

4.4 Dielectric loss determination of Green tape ${ }^{\mathrm{TM}} \ldots \ldots \ldots \ldots \ldots \ldots 78$ 


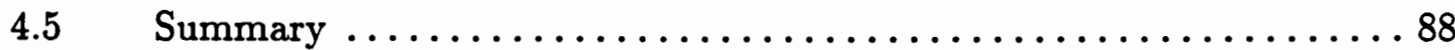

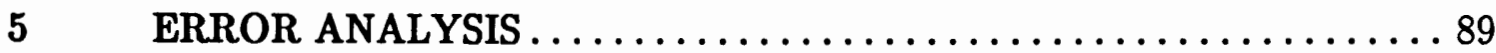

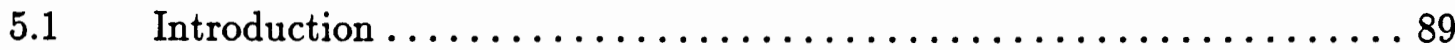

$5.2 \quad$ Measurement sensitivity $\ldots \ldots \ldots \ldots \ldots \ldots \ldots \ldots \ldots \ldots \ldots \ldots$

$5.3 \quad$ Geometrical error analysis $\ldots \ldots \ldots \ldots \ldots \ldots \ldots \ldots \ldots \ldots \ldots$

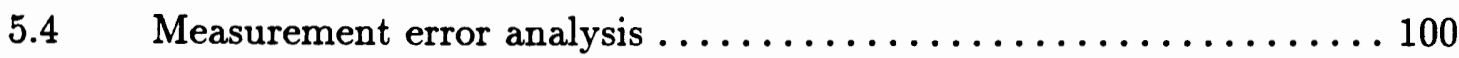

5.5 Perturbation technique error analysis............... 100

$5.6 \quad$ Perturbation technique limitations $\ldots \ldots \ldots \ldots \ldots \ldots \ldots \ldots \ldots \ldots$

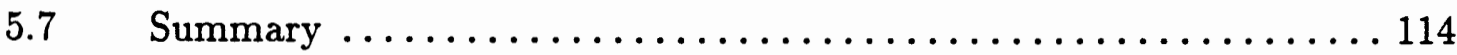

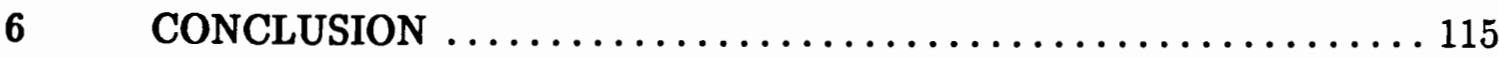

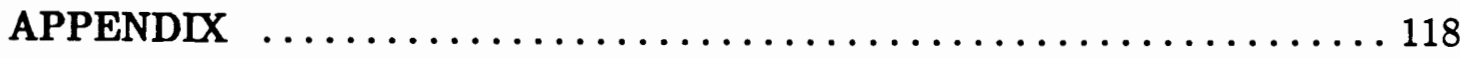

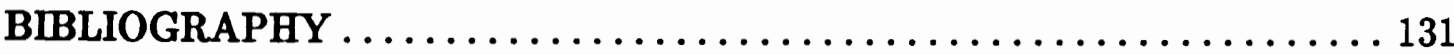

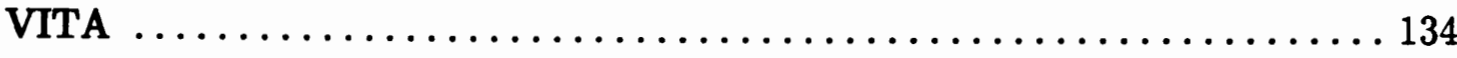




\section{CHAPTER 1}

\section{INTRODUCTION}

For high frequencies reaching into the microwave region, cavity structures are most appropriate for dielectric characterization. Traditionally, single frequency techniques that utilize cavity resonators are used. Unlike these methods, where the complex permittivity is derived from the quality factor of the cavity at the resonant frequency, the wideband dielectric-filled cavity technique [Saed, 1987; Riad, 1989; and Saed, 1990] measures the complex permittivity over a wide band of frequencies.

The cavity structure used in this technique is a reflection cavity, made with a conductor of known conductivity enclosing the dielectric. The dielectric is characterized by the dielectric constant and loss tangent. The cavity samples are fabricated to adapt to frequency domain measuring equipment. The reflection coefficient of a dielectric-filled cavity structure is measured and processed using a full field analysis to evaluate the dielectric properties based on knowledge of the sample geometry. This technique has been used successfully to estimate the dielectric constant. However limitations exist in the accuracy of estimating the dielectric loss factor. The loss estimated using this method yields a measure of the total cavity loss, including that of the conductor walls, while the field analysis is restricted to perfectly conducting walls leading to the inability to separate the dielectric loss estimate from the conductor loss. The conductor loss has a significant contribution to the total loss at microwave frequencies. The goal of this 
research is to expand the full field analysis applicable to two 3-dimensional regions coupled through an aperture in order to account for the conductor loss by perturbation to obtain a better estimate of the dielectric loss.

The separation of the conductor loss from the total loss will be done using perturbation [Harrington, 1961; Collin, 1960; and Van Bladel, 1964], incorporating the fields in the wideband dielectric-filled cavity found from the unperturbed cavity problem with perfectly conducting walls. The solution will be varied as a result of non-perfectly conducting boundary conditions causing the tangential component of the electric field to be non-zero at the conductor boundary. However, for good conductors the change in the tangential component of the magnetic field intensity is negligible and the basic fields in the cavity are only slightly "perturbed" from the loss-free solution.

To solve for the fields in the unperturbed problem, a general Green's function method, [Tai, 1972; Balanis, 1989; and Morse and Feshbak, 1953] is developed, in Chapter 2, to solve two 3-dimensional regions coupled through an aperture. The basis of the Green's function solution is the expansion of the Green's functions in terms of infinite series in the two regions of the structure. Because the capacity for treatment by computers is finite, the series have to be truncated. This Green's function solution will then be applied to the unperturbed problem with perfectly conducting walls. The series expansion coefficients are computed by using the method of moments [Harrington, 1968] to solve a matrix equation resulting enforcing continuity of the tangential fields over the aperture. The unperturbed analysis will establish a relationship between the reflection coefficient of the dielectric-filled cavity structure, the effective dielectric complex permittivity, 
and the cavity geometry.

Numerical convergence [Marcuvitz, 1951; Mittra and Lee, 1971; Mittra, 1963, 1972, 1975; and Leroy, 1983] is found to be related to both a minimum number of expansion terms and the ratio of the truncations in the two regions. The ratio aspect of convergence of the numerical results, is related to the relative truncation of the two Green's function expansions. The unique choice for the ratio of truncation is shown to be related to the geometrical dimensions of the structure. The solution convergence is studied by plotting the phase of the reflection coefficient versus the number of terms in the truncated expansions. The ratio of the truncation numbers, to ensure relative convergence, is determined. The minimum number of terms in the series expansions giving reasonable convergence in relative error is also determined. An additional verification of the unperturbed problem solution is obtained by comparing the aperture field continuity. The aperture fields computed with the minimum mode coefficients must have an excellent match [Lee, 1971].

The perturbation approach is developed in Chapter 3 to account for the conductor loss resulting from the imperfectly conducting cavity walls. The loss-free solution may be used to approximate the electric surface current at the conductor boundaries and to compute the power dissipated in the conductor. The total loss in the cavity will be computed from the measured reflection coefficient and the computed loss in the conductor walls will be separated from the total loss to estimate the dielectric loss.

As an example on the use of the developed technique, a typical problem will be simulated in Chapter 4. To further validate the use of the perturbation 
technique, experiments are presented for two low-loss dielectric materials. The obtained results will be compared to published results obtained by use of other cavity and transmission line techniques [Tyler \& Gasper 1989; Riad, 1991].

The perturbation technique has several potential sources of error, including sample geometry, frequency domain measurements, and numerical processing. The sensitivity of the estimated loss tangent of the dielectric under test is presented in Chapter 5 for the sample geometry, the measurement precision, and the assumed conductor conductivity using simulation.

Chapter 6 presents a summary and a conclusion, including applications and limitations of this work. Some recommendations for possible future work are also given. 
BLANK PAGE

(NO TEXT MISSING) 


\section{CHAPTER 2}

\section{THE UNPERTURBED PROBLEM}

\subsection{INTRODUCTION}

The unperturbed problem considered in this chapter is a reflection cavity structure designed to adapt to APC-7 connectors for frequency domain measuring instruments such as the HP $8510 \mathrm{~B}$ network analyzer. This cavity configuration is illustrated in Fig. 2.1. A full field analysis for this cavity structure, referred to as the wideband dielectric-filled cavity sample structure, is used for the evaluation of the dielectric properties of the dielectric material enclosed within it based on knowledge of the sample geometry and the measured S-parameters of the filled cavity. An iterative procedure is used to relate the measured reflection coefficient of the dielectric filled cavity and the complex dielectric permittivity, providing an estimate of the dielectric constant and the loss tangent.

In the full field analysis, electromagnetic equivalence principles are used to model the aperture separating the two regions of the structure: the transmission line region $(A)$ and the cavity region $(B)$ by equivalent magnetic currents. These magnetic surface currents are adjacent to a perfect conductor placed in the aperture as shown in Fig. 2.2. The magnetic surface current is expanded in terms of the transmission line modes and the resultant magnetic fields are matched at the aperture. 


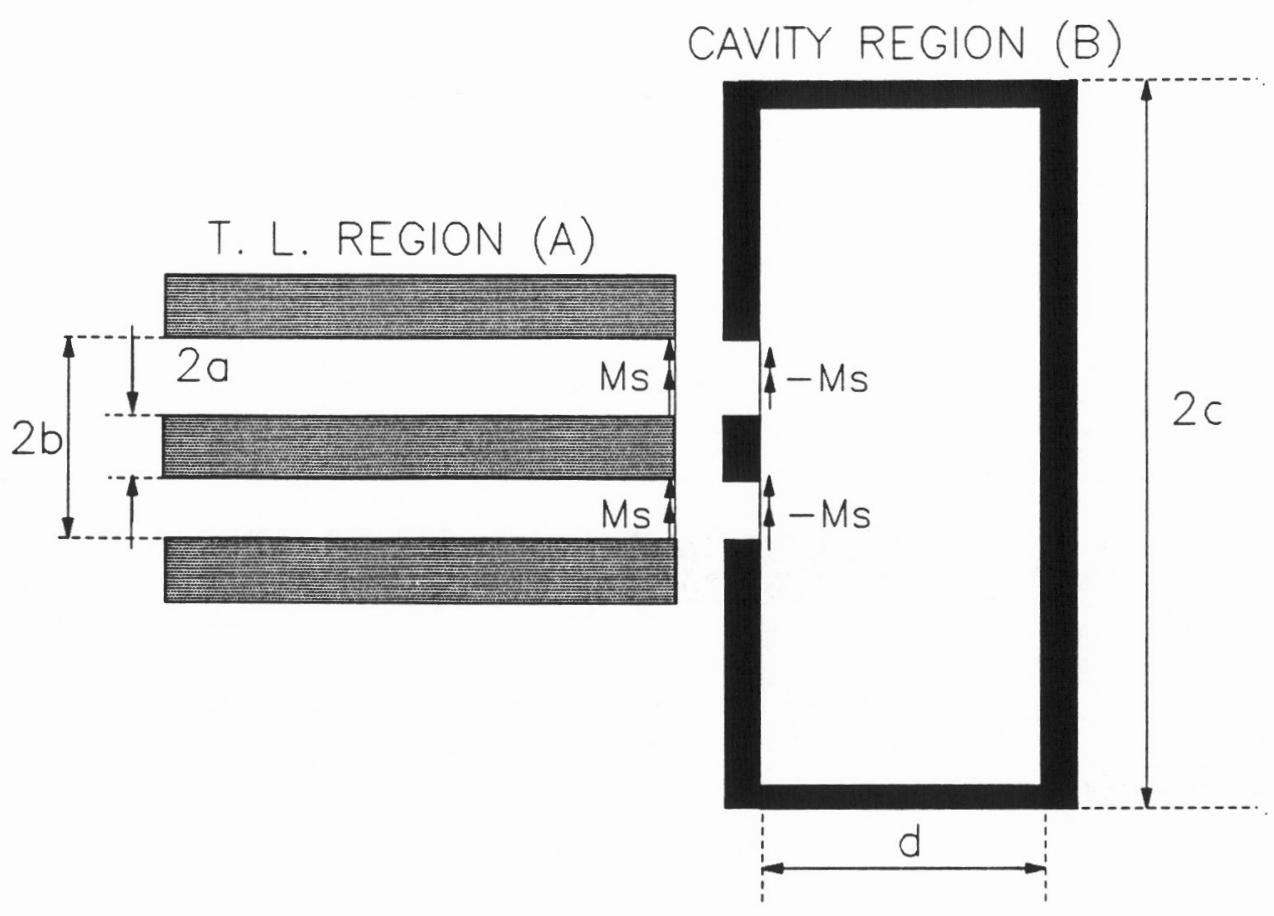

Figure 2.2. The equivalent problem. 


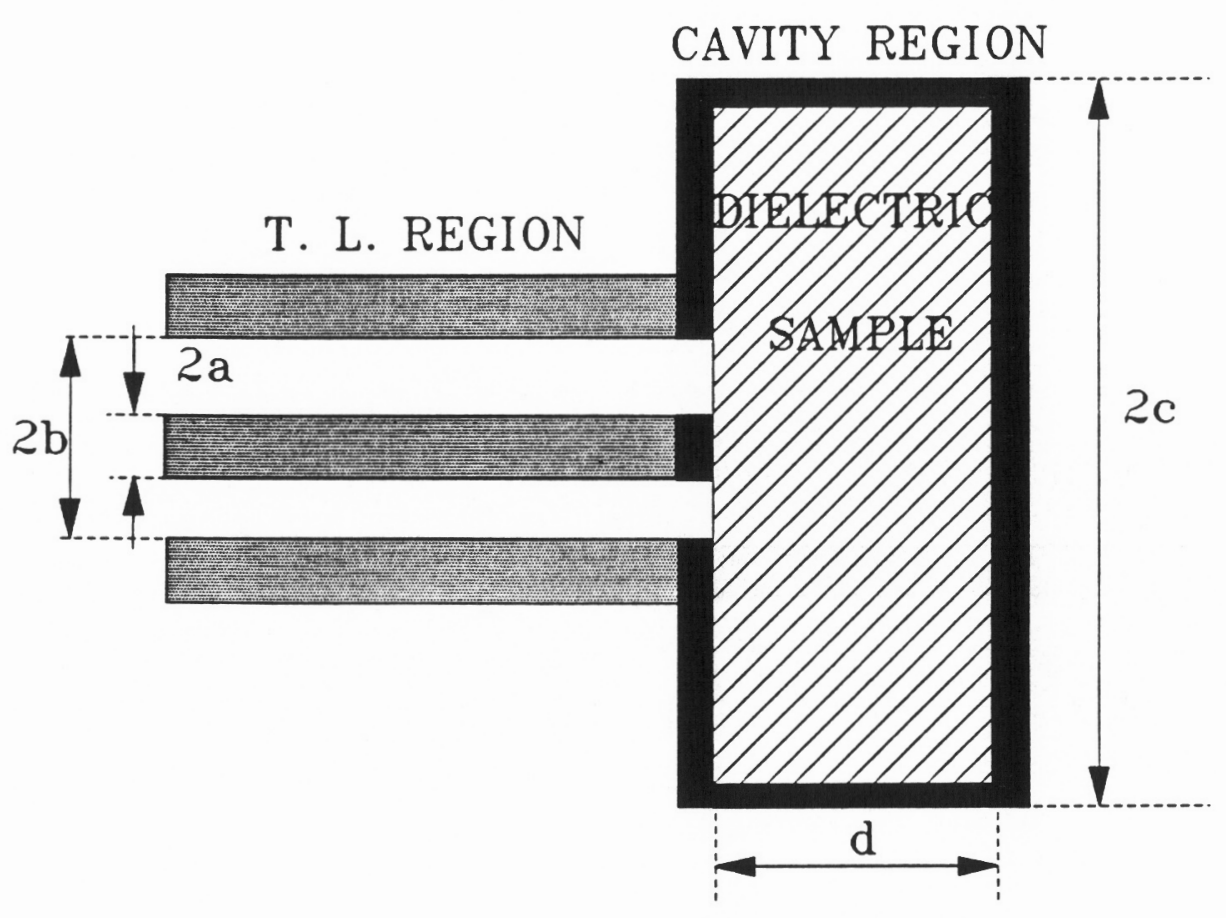

Figure 2.1. Reflection cavity configuration. 
This chapter develops the background of the unperturbed problem, paralle to the work of Saed [1987], using a more general approach. In this chapter, a basic Green's function solution approach will be developed for two coupled threedimensional regions. This approach will then be applied to the cavity structure of interest by expanding the Green's functions in both regions in terms of the modes of the structures respectively. Applying field continuity over the aperture or coupling surface, the method of moments will be used to solve for the magnetic current source imposed on the aperture, effectively applying the mode-matching method [Mittra \& Lee, 1971].

To examine the accuracy of the solution due to higher order modes, the fields will be computed in both regions for typical cavity dimensions and frequency. The fields at the aperture should agree from both regions, and the tangential electric field at the cavity walls should be identically zero. The number of terms retained in the series representation of the Green's function in the cavity region determines the limitations on the ability to match both the aperture fields and the zero electric field at the conductor walls. The relation between the number of modes in the transmission line region and the number of modes in the cavity region will be investigated in this work to ensure solution convergence.

\subsection{GENERAL GREEN'S FUNCTION APPROACH}

The basic Green's function problem is developed for two general three-dimensional regions. The resulting functions are then used to determine an equivalent aperture magnetic surface current between the two regions bounded by perfect conductors. 
To develop the basic Green's function problem, Maxwell's equations for a time dependence of the form $e^{j \omega t}$,in the presence of magnetic sources in a volume, will be stated as follows

$$
\begin{aligned}
& \nabla \times \bar{E}(\bar{r})=-\mathrm{j} \omega \mu_{0} \overline{\boldsymbol{H}}(\overline{\boldsymbol{r}})-\bar{M}(\overline{\boldsymbol{r}}) \\
& \nabla \times \overline{\boldsymbol{H}}(\overline{\boldsymbol{r}})=\mathrm{j} \omega \hat{\boldsymbol{\epsilon}} \overline{\boldsymbol{E}}(\overline{\boldsymbol{r}}) \\
& \nabla \cdot \bar{E}(\overline{\boldsymbol{r}})=0 \\
& \nabla \cdot \overline{\boldsymbol{H}}(\overline{\boldsymbol{r}})=\frac{-\nabla \cdot \bar{M}(\overline{\boldsymbol{r}})}{\mathrm{j} \omega \mu_{0}}
\end{aligned}
$$

where $\bar{E}(\bar{r})$ and $\bar{H}(\bar{r})$ represent the electric and magnetic field intensity, respectively, and $\bar{M}(\bar{r})$ represents the magnetic current source as functions of the position vector $(\bar{r})$. On the perfectly conducting walls bounding the two regions the tangential electric field vanishes and may be expressed as

$$
\hat{n} \times \bar{E}(\bar{r})=0
$$

where $\hat{n}$ denotes an outward unit vector normal to the surface. When approaching the aperture surface, the tangential electric field is equivalent to a magnetic surface current given by

$$
\hat{n} \times \bar{E}(\bar{r})=\bar{M}_{s}(\bar{r}) .
$$


Eliminating $\bar{E}(\bar{r})$ from Eq. (2.1), the vector Helmholtz wave equation in curl-curl form is obtained in the form

$$
\nabla \times \nabla \times \bar{H}(\bar{r})-k^{2} \bar{H}(\bar{r})=-j \omega \hat{\epsilon} \bar{M}(\bar{r})
$$

where $k^{2}=\omega^{2} \hat{\epsilon} \mu, \hat{\epsilon}$ being the complex medium permittivity, and $\mu$ equals $\mu_{0}$ in this case, the free-space permeability.

To solve Eq. (2.4) for $\overline{\boldsymbol{H}}(\overline{\boldsymbol{r}})$, the dyadic Green's function $\overline{\bar{G}}\left(\overline{\boldsymbol{r}}, \overline{\boldsymbol{r}}^{\prime}\right)$ was chosen and defined as the magnetic field due to a unit dyadic magnetic current source $\bar{I} \delta\left(\bar{r}-\bar{r}^{\prime}\right)$ satisfying the relation

$$
\nabla \times \nabla \times \overline{\bar{G}}\left(\overline{\boldsymbol{r}}^{\prime}, \overline{\boldsymbol{r}}\right)-k^{2} \overline{\bar{G}}\left(\overline{\boldsymbol{r}}^{\prime}, \overline{\boldsymbol{r}}\right)=-\mathrm{j} \omega \hat{\hat{\epsilon}} \overline{\bar{I}} \delta\left(\overline{\boldsymbol{r}}-\overline{\boldsymbol{r}}^{\prime}\right)
$$

The factor $\overline{\bar{I}}$ is the idem factor or unit dyad such that $\overline{\boldsymbol{A}} \bullet \overline{\bar{I}}=\overline{\bar{I}} \bullet \overline{\boldsymbol{A}}=\overline{\boldsymbol{A}}$ and $\nabla \cdot \overline{(I} \psi)=\nabla \psi$ and $\delta\left(\bar{r}-\bar{r}^{\prime}\right)$ is the three dimensional Dirac delta function ${ }^{1}$ in which $\bar{r}$ and $\bar{r}^{\prime}$ refer to the position vectors of the field and source points, respectively. The dyadic Green's function may also be defined to satisfy the appropriate boundary conditions for the considered problem.

Post-multiplying Eq. (2.4) by $\overline{\bar{G}}\left(\overline{\boldsymbol{r}}^{\prime}, \overline{\boldsymbol{r}}\right)$, pre-multiplying Eq. (2.5) by $\overline{\boldsymbol{H}}(\overline{\boldsymbol{r}})$, and subtracting the two resulting equations, the following expression may be

1 The Dirac delta function is not a function in the pure mathematical sense, but is properly defined as a generalized function [Lighthill, 1958] or a distribution [Stakgold, 1967]. 
obtained as

$$
\begin{aligned}
\overline{\boldsymbol{H}}(\overline{\boldsymbol{r}}) \bullet\left(\nabla \times \nabla \times \overline{\bar{G}}\left(\overline{\boldsymbol{r}}^{\prime}, \overline{\boldsymbol{r}}\right)\right)-(\nabla \times \nabla \times \overline{\boldsymbol{H}}(\overline{\boldsymbol{r}})) \cdot \overline{\bar{G}}\left(\overline{\boldsymbol{r}}^{\prime}, \overline{\boldsymbol{r}}\right) \\
=-\mathrm{j} \omega \hat{\epsilon} \overline{\boldsymbol{H}}(\overline{\boldsymbol{r}}) \cdot \overline{\bar{I}} \delta\left(\overline{\boldsymbol{r}}-\overline{\boldsymbol{r}}^{\prime}\right)+\mathrm{j} \omega \hat{\epsilon} \overline{\boldsymbol{M}}(\overline{\boldsymbol{r}}) \cdot \overline{\bar{G}}\left(\overline{\boldsymbol{r}}^{\prime}, \overline{\boldsymbol{r}}\right) .
\end{aligned}
$$

The vector dyadic Green's theorem [Van Bladel, 1964] given by the following expression

$$
\begin{array}{r}
\int_{V}\left\{\overline{\boldsymbol{H}}(\overline{\boldsymbol{r}}) \bullet\left(\nabla \times \nabla \times \overline{\bar{G}}\left(\boldsymbol{r}^{\prime}, \overline{\boldsymbol{r}}\right)-(\nabla \times \nabla \times \overline{\boldsymbol{H}}(\overline{\boldsymbol{r}})) \cdot \overline{\bar{G}}\left(\overline{\boldsymbol{r}}^{\prime}, \overline{\boldsymbol{r}}\right)\right)\right\} d v \\
=\oint_{S}\left\{\overline{\boldsymbol{H}}(\overline{\boldsymbol{r}}) \bullet \hat{\boldsymbol{n}} \times\left(\nabla \times \overline{\bar{G}}\left(\overline{\boldsymbol{r}}^{\prime}, \overline{\boldsymbol{r}}\right)\right)\right. \\
\left.+(\hat{\boldsymbol{n}} \times \nabla \times \overline{\boldsymbol{B}}(\overline{\boldsymbol{r}})) \bullet \overline{\bar{G}}\left(\overline{\boldsymbol{r}}^{\prime}, \overline{\boldsymbol{r}}\right)\right\} d s,
\end{array}
$$

may be used with $\hat{n}$ denoting the outward unit-vector normal to the surface $S$ enclosing the volume $V$, in order to obtain an expression for $\bar{H}(\bar{r})$. Integrating Eq. (2.6) over the volume $V$ and using Eq. (2.7), one can obtain

$$
\begin{aligned}
& \oint_{S}\left\{\overline{\boldsymbol{H}}(\overline{\boldsymbol{r}}) \bullet \hat{\boldsymbol{n}} \times\left(\nabla \times \overline{\bar{G}}\left(\overline{\boldsymbol{r}}^{\prime}, \overline{\boldsymbol{r}}\right)\right)\right. \\
&\left.-(\hat{\boldsymbol{n}} \times \nabla \times \overline{\boldsymbol{H}}(\overline{\boldsymbol{r}})) \cdot \overline{\bar{G}}\left(\overline{\boldsymbol{r}}^{\prime}, \overline{\boldsymbol{r}}\right)\right\} d s \\
&=-\mathrm{j} \omega \hat{\epsilon} \overline{\boldsymbol{H}}\left(\overline{\boldsymbol{r}}^{\prime}\right)+\mathrm{j} \omega \hat{\hat{\epsilon}} \int_{V} \bar{M}(\overline{\boldsymbol{r}}) \cdot \overline{\bar{G}}\left(\overline{\boldsymbol{r}}^{\prime}, \overline{\boldsymbol{r}}\right) d v
\end{aligned}
$$


The surface integral in Eq. (2.8) may be simplified using Eq. (2.1b) in conjunction with Eq. (2.2) and Eq. (2.3) to specify the boundary conditions on $\overline{\boldsymbol{H}}$ and imposing a boundary condition on the Green's function

$$
\hat{n} \times \nabla \times \overline{\bar{G}}\left(\bar{r}, \bar{r}^{\prime}\right)=0
$$

for $\bar{r}$ on the surface $S$, to obtain the expression

$$
-\int_{A}\left\{\overline{\boldsymbol{M}}_{\boldsymbol{s}}(\overline{\boldsymbol{r}}) \cdot \overline{\bar{G}}\left(\overline{\boldsymbol{r}}^{\prime}, \overline{\boldsymbol{r}}\right)\right\} d s=-\overline{\boldsymbol{H}}\left(\overline{\boldsymbol{r}}^{\prime}\right)+\int_{V}\left\{\overline{\boldsymbol{M}}(\overline{\boldsymbol{r}}) \cdot \overline{\bar{G}}\left(\overline{\boldsymbol{r}}^{\prime}, \overline{\boldsymbol{r}}\right)\right\} d v
$$

where $A$ is the aperture surface. The source $\bar{M}$ is typically related to an independent source creating a source field for the problem. This source will give rise to both incident and reflected fields from a closed aperture. Thus Eq.(2.10) may be rewritten as

$$
\overline{\boldsymbol{H}}(\overline{\boldsymbol{r}})=\int_{V}\left\{\overline{\boldsymbol{M}}\left(\overline{\boldsymbol{r}}^{\prime}\right) \cdot \overline{\bar{G}}\left(\overline{\boldsymbol{r}}, \overline{\boldsymbol{r}}^{\prime}\right)\right\} d v+\int_{A}\left\{\overline{\boldsymbol{M}}_{\boldsymbol{s}}\left(\overline{\boldsymbol{r}}^{\prime}\right) \cdot \overline{\bar{G}}\left(\bar{r}, \overline{\boldsymbol{r}}^{\prime}\right)\right\} d s^{\prime}
$$

where $\bar{r}$ and $\bar{r}^{\prime}$ have been interchanged. One may define the field due to the surface magnetic current $\overline{\boldsymbol{M}}_{\boldsymbol{s}}\left(\overline{\boldsymbol{r}}^{\prime}\right)$ by $\overline{\boldsymbol{H}}_{\boldsymbol{s}}(\overline{\boldsymbol{T}})$ as

$$
\overline{\boldsymbol{H}}_{\boldsymbol{s}}(\overline{\boldsymbol{r}})=\int_{A}\left\{\overline{\boldsymbol{M}}_{\boldsymbol{s}}\left(\overline{\boldsymbol{r}}^{\prime}\right) \cdot \overline{\bar{G}}\left(\overline{\boldsymbol{r}}, \overline{\boldsymbol{r}}^{\prime}\right)\right\} d s^{\prime}
$$

To solve the coupled two-region problem, one can first observe that

$$
\overline{\boldsymbol{M}}_{\boldsymbol{s}}^{B}\left(\overline{\boldsymbol{r}}^{\prime}\right)=-\overline{\boldsymbol{M}}_{\boldsymbol{s}}^{A}\left(\overline{\boldsymbol{r}}^{\prime}\right)
$$


due to the negation of the normal unit vector in the aperture for the two problems. The superscripts $A$ and $B$ denote the two different regions. The use of such a current relationship automatically enforces the continuity of the tangential electric field intensity in the aperture region. To determine this magnetic surface current, one imposes continuity on the tangential magnetic field intensity at the aperture.

\subsection{SOLUTION FOR THE GREEN'S FUNCTION}

To relate the two general regions used in the previous section to the specific problem of this dissertation, the transmission line region is defined as region $A$, and the cavity region as region $B$ as shown in Fig. 2.2. The structures of the two regions and the source are axially symmetric; therefore there is no $z$-component of magnetic field, and only TM higher order modes are generated due to the discontinuity at the aperture. The magnetic fields are $\phi$-directed and are dependent only on $\rho$ and $z$. In order to solve for the magnetic field in a volume, due to a magnetic surface current, one may use the expression

$$
\overline{\boldsymbol{H}}_{\boldsymbol{s}}(\overline{\boldsymbol{r}})=\int_{A}\left\{\overline{\boldsymbol{M}}_{\boldsymbol{s}}\left(\overline{\boldsymbol{r}}^{\prime}\right) \cdot \overline{\bar{G}}\left(\overline{\boldsymbol{r}}, \overline{\boldsymbol{r}}^{\prime}\right)\right\} d s^{\prime}
$$

which requires the definition of the Green's function $\overline{\bar{G}}\left(\bar{r}, \bar{r}^{\prime}\right)$. The magnetic field $\bar{H}(\overline{\boldsymbol{\tau}})$ and the magnetic surface current $\overline{\boldsymbol{M}}_{\boldsymbol{s}}\left(\overline{\boldsymbol{\tau}}^{\prime}\right)$ are both independent of $\phi$ except for the direction-dependence. For the $\phi$-independent field and source, Eq. (2.14) may be rewritten as

$$
\overline{\boldsymbol{B}}_{s}(\overline{\boldsymbol{r}})=\int_{A}\left\{M_{s}\left(\rho^{\prime}\right) \hat{\phi}^{\prime} \cdot \overline{\bar{G}}\left(\overline{\boldsymbol{r}}, \overline{\boldsymbol{r}}^{\prime}\right)\right\} d \phi^{\prime} \rho^{\prime} d \rho^{\prime}
$$


By defining

$$
\int_{0}^{2 \pi} \hat{\phi}^{\prime} \cdot \overline{\bar{G}}\left(\bar{r}, \bar{r}^{\prime}\right) d \phi^{\prime}=\bar{g}\left(\rho, \rho^{\prime}\right),
$$

a solution for $\bar{H}_{s}(\rho)$ may be obtained of the form

$$
\overline{\boldsymbol{H}}_{s}(\rho)=\int_{a}^{b}\left\{M_{\mathcal{S}}\left(\rho^{\prime}\right) \overline{\boldsymbol{g}}\left(\rho, \rho^{\prime}\right)\right\} \rho^{\prime} \mathrm{d} \rho^{\prime} .
$$

In order to obtain $\bar{g}\left(\rho, \rho^{\prime}\right)$, one revert to Eq. (2.5) given as

$$
\nabla^{\prime} \times \nabla^{\prime} \times \overline{\bar{G}}\left(\bar{r}, \bar{r}^{\prime}\right)-k^{2} \overline{\bar{G}}\left(\bar{r}, \bar{r}^{\prime}\right)=-j \omega \hat{\bar{\epsilon}} \overline{\bar{I}} \delta\left(\bar{r}, \bar{r}^{\prime}\right)
$$

with the boundary condition given in Eq. (2.9) as

$$
\hat{n} \times \nabla^{\prime} \times \overline{\bar{G}}\left(\bar{r}, \bar{r}^{\prime}\right)=0
$$

By taking the anterior product of Eq. (2.18) with $\hat{\phi}^{\prime}$, expressing the delta distribution $\delta\left(\bar{r}-\bar{r}^{\prime}\right)$ in cylindrical coordinates [Balanis, 1989], and integrating over $\phi^{\prime}$ from 0 to $2 \pi$, the following expression may be obtained

$$
\begin{aligned}
\int_{\phi^{\prime}}\left\{\hat{\phi}^{\prime} \cdot\left[\nabla^{\prime} \times \nabla^{\prime} \times \overline{\bar{G}}\left(\overline{\boldsymbol{r}}, \overline{\boldsymbol{r}}^{\prime}\right)\right]-k^{2} \hat{\phi}^{\prime} \cdot \overline{\bar{G}}\left(\bar{r}, \overline{\boldsymbol{r}}^{\prime}\right)\right\} d \phi^{\prime} \\
=-\int_{\phi^{\prime}} \mathrm{j} \omega \hat{\epsilon} \hat{\phi}^{\prime} \cdot \overline{\bar{I}} \delta\left(\rho-\rho^{\prime}\right) \delta\left(\phi-\phi^{\prime}\right) \delta\left(z-z^{\prime}\right) d \phi^{\prime} .
\end{aligned}
$$


Because of the periodicity of the function involved, the integration over $\phi^{\prime}$ of the $\phi^{\prime}$-derivatives will drop out. The resultant expression from Eq. (2.20) may be rewritten by using the definition in Eq. (2.16) as

$$
\frac{\partial^{2}}{\partial z^{\prime 2}} \bar{g}+\frac{\partial}{\partial \rho^{\prime}}\left(\frac{1}{\rho^{\prime}} \frac{\partial}{\partial \rho^{\prime}} \rho^{\prime} \bar{g}\right)+k^{2} \bar{g}=\frac{j \omega \hat{\epsilon}}{\rho^{\prime}} \bar{\phi}^{\prime} \delta\left(\rho-\rho^{\prime}\right) \delta\left(z-z^{\prime}\right)
$$

The Green's function $\bar{g}$ may further be writen as $\bar{g}=g \hat{\phi}^{\prime}$, for the circular symmetrical problem under consideration. The resultant, scalar Green's function $g$ must satisfy the scalar form of Eq. (2.19). The function $g$ may be expanded in a modal series for each region of interest. For a specific mode of the series, the double $z$-derivative may be replaced by $\left(-k_{z}^{2} g\right)$ in order to define $k_{\rho}^{2}=k^{2}-k_{z}^{2}$ for the guided wave structures. Eq. (2.21) may then be rewritten as

$$
\frac{\partial}{\partial \rho^{\prime}}\left(\frac{1}{\rho^{\prime}} \frac{\partial}{\partial \rho^{\prime}} \rho^{\prime} g\right)+k_{\rho}^{2} g=0
$$

for $z^{\prime}$ not equal $z$. The solution to Eq. (2.22) may be written as a Bessel function of the first order in the form

$$
g\left(\rho^{\prime}, z^{\prime} ; \rho, z\right)=A(\rho, z) \mathrm{B}_{1}\left(k_{\rho} \rho^{\prime}\right) h\left(k_{z} z^{\prime}\right)
$$

where $B_{1}$ is a general linear combination of two Bessel functions of order one, $h$ is the related harmonic behavior in $z^{\prime}$, and $A(\rho, z)$ is a constant coefficient with respect to $\rho^{\prime}$ and $z^{\prime}$.

The function $g$ must satisfy the boundary condition resulting from combining the boundary condition given by Eq. (2.19) and the function $g$ given by 
Eq. (2.16) to give

$$
\int_{0}^{2 \pi} \hat{\phi}^{\prime} \bullet \hat{z} \times\left(\nabla^{\prime} \times \overline{\bar{G}}\left(\bar{r}, \bar{r}^{\prime}\right)\right) d \phi^{\prime}=0
$$

on the perfect-electric conducting surface covering the two regions. By performing the curl and taking the $\phi^{\prime}$-component of Eq. (2.24), the Newman boundary conditions in $z^{\prime}$ on the function $g$ may be stated as

$$
\frac{\partial}{\partial z^{\prime}} g=0
$$

At the $z$-plane closing the left portion of the transmission line region, an absorbing boundary condition will be used instead. At the radial boundaries, a similar process gives

$$
\frac{\partial}{\partial \rho^{\prime}}\left(\rho^{\prime} g\right)=0
$$

Applying the boundary condition stated in Eq. (2.25) in addition to an absorbing boundary condition as $z^{\prime} \rightarrow-\infty$ for the transmission line region $A$, the $z^{\prime}$-dependent part of $g$ may be expressed as

$$
h\left(z^{\prime}\right)= \begin{cases}C e^{\mathrm{j} k_{z} z^{\prime}} & z^{\prime}<z \\ D \cos k_{z} z^{\prime} & z^{\prime}>z\end{cases}
$$

To satisfy the boundary condition of Eq. (2.26) at the radius a, the Bessel function $\mathrm{B}_{1}$ given as a linear combination of the first and second kind Bessel functions has 
the form

$$
\mathrm{B}_{1}\left(k_{\rho} \rho^{\prime}\right)=N_{0}\left(k_{\rho} a\right) J_{1}\left(k_{\rho} \rho^{\prime}\right)-J_{d}\left(k_{\rho} a\right) N_{1}\left(k_{\rho} \rho^{\prime}\right)
$$

for any $a \leq \rho^{\prime} \leq b$, where $a$ and $b$ are the inner and outer radii of the transmission line region, respectively. To satisfy Eq. (2.26) at $\rho^{\prime}=b, k_{\rho}$ must satisfy the condition

$$
N_{d}\left(k_{\rho} a\right) J_{0}\left(k_{\rho} b\right)-J_{d}\left(k_{\rho} a\right) N_{d}\left(k_{\rho} b\right)=0 .
$$

Approaching the source plane $\left(z^{\prime}=z\right)$, the continuity requirements on $g$ from the scalar form of Eq. (2.21) may be obtained. This continuity initially requires $g$ to be continuous as expressed by the condition

$$
\left.g\right|_{z^{\prime}=z(-)}=\left.g\right|_{z^{\prime}=z(+)}
$$

where the signs $(-)$ and $(+)$ mean the value of $z^{\prime}$ approaching $z$ from the left and right sides, respectively. The function $g$ may be expanded as a series over all the possible modes of the structure as

$$
g\left(\rho^{\prime}, z^{\prime} ; \rho, z\right)= \begin{cases}\sum_{n} C_{n}(\rho, z) \mathrm{B}_{1}\left(k_{\rho_{n}} \rho^{\prime}\right) e^{\mathrm{j} k_{z_{n}} z}, & z^{\prime}<z \\ \sum_{n} D_{n}(\rho, z) \mathrm{B}_{1}\left(k_{\rho_{n}} \rho^{\prime}\right) \cos k_{z_{n}} z, & z^{\prime}>z\end{cases}
$$

Applying the condition of Eq. (2.30), and using the orthogonality of the Bessel 
functions, the following expression may be obtained

$$
C_{n} e^{\mathrm{j} k_{z_{n}} z}=D_{n} \cos k_{z_{n}} z
$$

Also at the source plane $\left(z=z^{\prime}\right)$, the $z^{\prime}$-derivative of the Green's function must be discontinuous by $\left(j \omega \hat{\epsilon} / \rho^{\prime}\right) \delta\left(\rho-\rho^{\prime}\right)$ in order to satisfy Eq. (2.21). This condition may be stated as

$$
\left.\frac{\partial g}{\partial z^{\prime}}\right|_{z^{\prime}=z_{(+)}}-\left.\frac{\partial g}{\partial z^{\prime}}\right|_{z^{\prime}=z_{(-)}}=\frac{\mathrm{j} \omega \hat{\epsilon}}{\rho^{\prime}} \delta\left(\rho-\rho^{\prime}\right)
$$

Applying this condition to the Green's function, one may write

$$
\sum_{n} F_{n}\left[N_{d}\left(k_{\rho_{n}} a\right) J_{1}\left(k_{\rho_{n}} \rho^{\prime}\right)-J_{d}\left(k_{\rho_{n}} a\right) N_{1}\left(k_{\rho_{n}} \rho^{\prime}\right)\right]=\frac{\delta\left(\rho-\rho^{\prime}\right)}{\rho^{\prime}}
$$

where $F_{n}$ are defined as

$$
F_{n}=\left(-k_{z_{n}} D_{n} \sin k_{z_{n}} z-\mathrm{j} k_{z_{n}} C_{n} e^{\mathrm{j} k_{z_{n}} z}\right) / \mathrm{j} \omega \hat{\epsilon}
$$

To determine $F_{n}$, both sides of Eq. (2.34) may be multiplied by $\mathrm{B}_{1}\left(k_{\rho_{m}}\right)$, then the resultant equation may be integrated over the variable $\rho^{\prime}$ from $a$ to $b$ to give the expression

$$
\int_{a}^{b} \sum_{n} F_{n} \rho^{\prime} \mathrm{B}_{1}\left(k_{\rho_{n}} \rho^{\prime}\right) \mathrm{B}_{1}\left(k_{\rho_{m}} \rho^{\prime}\right) d \rho^{\prime}=\int_{a}^{b} \mathrm{~B}_{1}\left(k_{\rho_{m}} \rho^{\prime}\right) \delta\left(\rho-\rho^{\prime}\right) d \rho^{\prime} .
$$

Using the orthogonality of the Bessel's functions satisfying the boundary 
conditions, one may solve for $F_{n}$ to obtain

$$
F_{n}=\frac{2 \mathrm{~B}_{1}\left(k_{\rho_{n}} \rho\right)}{b^{2} \mathrm{~B}_{1}^{2}\left(k_{\rho_{n}} b\right)-a^{2} \mathrm{~B}_{1}^{2}\left(k_{\rho_{n}} a\right)} .
$$

Now one may solve for the coefficients $C_{n}$ and $D_{n}$ from Eq. (2.35) to give

$$
C_{n}=-\frac{\omega \hat{\epsilon} F_{n}}{k_{z_{n}}} e^{\mathrm{j} k_{z_{n}} z}
$$

and

$$
D_{n}=-\frac{\omega \hat{\epsilon} F_{n}}{k_{z_{n}}} \cos k_{z_{n}} z
$$

With the magnetic current source located at the aperture $\left(z^{\prime}=0\right)$, the required function $g^{A}$, according to Eqs. (2.31), (2.38), and (2.37), may be written as

$$
g^{A}\left(\rho, z ; \rho^{\prime}, 0\right)=\sum_{n} \frac{-2 \omega \epsilon_{0}}{k_{z_{n}}^{A}} \frac{\mathrm{B}_{1}\left(k_{\rho_{n}}^{A} \rho\right) B_{1}\left(k_{\rho_{n}}^{A} \rho^{\prime}\right)}{b^{2} \mathrm{~B}_{1}^{2}\left(k_{\rho_{n}}^{A} b\right)-a^{2} \mathrm{~B}_{1}^{2}\left(k_{\rho_{n}}^{A} a\right)} e^{\mathrm{j} k_{z_{n}}^{A} z}
$$

where $\mathrm{B}_{1}$ is given by Eq. $(2.28)$, and $n=0,1 \ldots . . \infty$. The coefficients $k_{\rho_{n}}^{A}$ represent the solutions to Eq. (2.29) and $k_{z_{n}}^{A}$ are found using the equation

$$
\left(k_{\mathrm{z}_{n}}^{A}\right)^{2}=\left(\omega^{2} \epsilon_{0} \mu_{0}\right)^{2}-\left(k_{\rho_{n}}^{A}\right)^{2} .
$$

The complex permittivity in the development of the Green's function has been substituted in Eqs. (2.40) and (2.41) with the free space permittivity since the transmission line region is considered. 
Following the same procedure for the cavity region $B$, to solve for the scalar Green's function, the required function $g^{B}$ may be obtained as

$$
g^{B}\left(\rho, z ; \rho^{\prime}, 0\right)=\sum_{m} \frac{-2 \mathrm{j} \omega \hat{\epsilon}}{k_{z_{m}}^{B}} \frac{J_{1}\left(k_{\rho_{m}}^{B} \rho\right) J_{1}\left(k_{\rho_{m}}^{B} \rho^{\prime}\right)}{c^{2} J_{1}^{2}\left(k_{\rho_{m}}^{B} c\right) \sin \left(k_{z_{m}}^{B} d\right)} \cos k_{z_{m}}^{B}(z-d),
$$

where $c$ is the cavity radius, $d$ is the cavity thickness and $m=1,2 \ldots \infty$. The coefficients $k_{\rho_{m}}^{B}$ must satisfy the condition

$$
J_{0}\left(k_{\rho_{m}}^{B} c\right)=0
$$

and $k_{z_{m}}^{B}$ must satisfy

$$
k_{z_{m}}^{B}=\sqrt{\omega^{2} \hat{\epsilon} \mu_{0}-\left(k_{\rho_{m}}^{B}\right)^{2}} .
$$

\subsection{SOLUTION TO THE UNPERTURBED PROBLEM}

Assuming each region is a guided wave structure of arbitrary cross-section, the Green's functions may be expanded in terms of the orthogonal modes of the two structures as shown in Eqs. (2.40) and (2.42). In region $A$, there is an incident magnetic field $\overline{\boldsymbol{H}}^{i}(\overline{\boldsymbol{r}})$, a reflected magnetic field from the closed aperture $\overline{\boldsymbol{H}}^{\boldsymbol{r}}(\overline{\boldsymbol{r}})$, and a scattered field $\bar{H}_{s}^{A}(\bar{r})$ due to the equivalent magnetic current source $\bar{M}_{s}(\bar{r})$, imposed in front of the aperture. In region $B$, there is only the scattered field $\overline{\boldsymbol{H}}_{\boldsymbol{s}}^{B}(\overline{\boldsymbol{r}})$ due to the surface magnetic current source $\left(-\overline{\boldsymbol{M}}_{\boldsymbol{s}}(\overline{\boldsymbol{r}})\right)$. Due to the continuity over the aperture, the tangential magnetic field intensities of the two regions must 
be equal when approaching the aperture as shown by the expression

$$
\overline{\boldsymbol{H}}^{i}(\overline{\boldsymbol{r}})+\overline{\boldsymbol{B}}^{r}(\overline{\boldsymbol{r}})+\overline{\boldsymbol{H}}_{\mathcal{S}}^{A}(\overline{\boldsymbol{r}})=\overline{\boldsymbol{H}}_{\boldsymbol{S}}^{B}(\overline{\boldsymbol{r}}), \quad \text { at } z=0
$$

where the $s$ subscript denotes terms due to the aperture magnetic current. The incident field in the transmission line region is assumed to be a TEM mode. At $z=0$, both the incident and reflected fields are equal and given by

$$
H^{i}(\rho)=H^{r}(\rho)=\frac{1}{\rho \sqrt{\eta_{o} \pi \ln (b / a)}}
$$

where $\eta_{0}$ is the free space intrinsic impedance given by $\sqrt{\frac{\mu_{0}}{\epsilon_{0}}}$. This incident field has been normalized for unity incident power. The magnetic current $\bar{M}_{\boldsymbol{s}}(\overline{\boldsymbol{r}})$ is a surface current present at the aperture and may be be expressed as a function of its transverse position as

$$
\bar{M}_{\boldsymbol{s}}(\overline{\boldsymbol{r}})=M_{s}\left(\rho^{\prime}\right) \hat{\phi}^{\prime}
$$

To evaluate the scattered fields in both regions $A$ and $B$, Eq. (2.16) will be used to obtain the $\phi^{\prime}$ - directed scattered fields at a plane approaching the $z=0$ plane, as

$$
H_{s}^{A}(\rho)=\int_{a}^{b}\left\{M_{s}\left(\rho^{\prime}\right) g^{A}\left(\rho, \rho^{\prime}\right)\right\} \rho^{\prime} \mathrm{d} \rho^{\prime},
$$

and

$$
H_{s}^{B}(\rho)=-\int_{a}^{b}\left\{M_{s}\left(\rho^{\prime}\right) g^{B}\left(\rho, \rho^{\prime}\right)\right\} \rho^{\prime} \mathrm{d} \rho^{\prime}
$$


Substituting the appropriate Green's function as given by Eq. (2.40) in Eq. (2.48), one may obtain

$$
H_{s}^{A}(\rho)=-\sum_{n=0} A_{n} h_{n}(\rho) / \eta_{n}
$$

where the coefficients $A_{n}$, and $\eta_{n}$ may be defined as

and

$$
A_{n}=2 \pi \int_{a}^{b} M_{s}\left(\rho^{\prime}\right) h_{n}\left(\rho^{\prime}\right) \rho^{\prime} d \rho^{\prime},
$$

$$
\eta_{n}=\frac{k_{z_{n}}^{A}}{\omega \epsilon_{0}}
$$

The functions $h_{n}$ may be defined as

$$
h_{n}(\rho)=\frac{\mathrm{B}_{1}\left(k_{\rho_{n}}^{A} \rho\right)}{\sqrt{\pi\left[b^{2} \mathrm{~B}_{1}^{2}\left(k_{\rho_{n}}^{A} b\right)-a^{2} \mathrm{~B}_{1}^{2}\left(k_{\rho_{n}}^{A} a\right)\right]}}
$$

where $a$ and $b$ are the transmission line radii, and $\mathrm{B}_{1}\left(k_{\rho_{n}}^{A} \rho\right)$ are the Bessel functions. For the cavity region, the expression

$$
H_{s}^{B}(\rho)=\mathrm{j} \omega \hat{\epsilon} \sum_{m=1} \frac{C_{m}}{k_{z_{m}}^{B}} J_{1}\left(k_{\rho_{m}}^{B} \rho\right) \cos \left(k_{z_{m}}^{B} d\right)
$$

may be obtained from Eqs. (2.49) and (2.42), where the coefficients $C_{m}$ may be defined as

$$
C_{m}=\frac{-2}{c^{2} J_{1}^{2}\left(k_{\rho_{m}}^{B} c\right) \sin \left(k_{z_{m}}^{B} d\right)} \int_{a}^{b} M_{s}\left(\rho^{\prime}\right) J_{1}\left(k_{\rho_{m}}^{B} \rho^{\prime}\right) \rho^{\prime} d \rho^{\prime}
$$


where $c$ is the cavity radius, and $d$ is its thickness. For computational purposes, the two infinite series in Eqs. (2.50) and (2.54) will be truncated to $N$ modes in region $A$ and $M$ modes in region $B$.

$M_{s}$ may be expanded in terms of a set of functions $M_{i}$ which span the space of $M_{s}$. This expansion is given by

$$
M_{s}\left(\rho^{\prime}\right)=\sum_{i} V_{i} M_{i}\left(\rho^{\prime}\right)
$$

The modes of the transmission line region provide a reasonable choice for expansion with the same number of expansion terms as

$$
M_{n}(\rho)=h_{n}(\rho)
$$

For an exact solution, Eq. (2.56) is an infinite summation and the $M_{n}$ form a complete set of basis functions. For an approximate solution, the series is truncated to a finite number of terms which corresponds to the number of modes in the transmission line region $(N)$. Substituting with Eqs. (2.46) and (2.56) in Eq. (2.45), and using linearity, the following expression may be obtained as

$$
2 H^{i}-\sum_{n} V_{n} H_{s}^{A}\left(M_{n}\right)-\sum_{n} V_{n} H_{s}^{B}\left(M_{n}\right)=0
$$

where $H_{s}^{A}\left(M_{n}\right)$, and $H_{s}^{B}\left(M_{n}\right)$ are the tangential fields due to the current expansion function $M_{n}$. These fields are the same as in Eqs. (2.50) and (2.54), except that the coefficients $A_{n}$ and $C_{m}$ will have another subscript to denote the current expansion. 
These coefficients may be given by

and

$$
A_{n i}=2 \pi \int_{a}^{b} M_{i}\left(\rho^{\prime}\right) h_{n}\left(\rho^{\prime}\right) \rho^{\prime} d \rho^{\prime}, \quad \quad \text { for } \quad n=i=0,1,2, \ldots N-1
$$

$$
C_{m n}=\frac{-2}{c^{2} J_{1}^{2}\left(k_{\rho_{m}}^{B} c\right) \sin \left(k_{z_{m}}^{B} d\right)} \int_{a}^{b} M_{n}\left(\rho^{\prime}\right) J_{1}\left(k_{\rho_{m}}^{B} \rho^{\prime}\right) \rho^{\prime} d \rho^{\prime}, \text { for } m=1,2, \ldots M
$$

The method of moments [Harrington, 1968] is then used to solve Eq. (2.58), to obtain the current coefficients $V_{n}$, from which the magnetic current source is constructed.

The reflection coefficient of the cavity structure may be determined from the resultant mode coefficients. The apparent complex permittivity of the dielectric is determined from the reflection coefficient of the structure using an iterative procedure to optimize the fit of the measured frequency response of the reflection structure to the simulation results. A program to perform this computation is provided in [Saed, 1987].

\subsection{APERTURE FIELDS COMPARISON AND MODE DETERMINATION}

In the existing technique, the problem involves a discontinuity at the aperture separating two regions. The fields in these two regions have been expanded in terms of an infinite number of discrete sets of modes with unknown coefficients. The numerical problem theoretically involves the calculation of these amplitude coefficients from an an infinite system using the method of moments. Because the capacity for treatment by computers is limited, the series used in 
Section 2.3 have been truncated. These series truncations may potentially introduce significant errors in the computed fields and the resultant reflection coefficient. In order to determine the effects of truncation error, the field and the reflection coefficient convergences were studied as functions of the series truncations. Prior to investigating the convergence of the reflection coefficient, the goodness-of-fit of the electric and magnetic field continuity at the aperture will be studied.

\subsubsection{Aperture Magnetic Field}

As a check on the accuracy of the solution computed from the higher order modes, the tangential magnetic field at the aperture will be computed and plotted in both the transmission line region and the cavity region.

The magnetic field in the transmission line region, tangential to the aperture, is represented by

$$
H^{A}=-\sum_{n=0}^{N-1} \frac{V_{n}}{\eta_{n}} h_{n}(\rho)
$$

where the coefficients $V_{n}$ are the expansion coefficients of the magnetic current distribution on the aperture $M_{s}$ in terms of the expansion functions $M_{n}$. These coefficients $V_{n}$ are found by solving the matrix equation in Eq. (2.58). The functions $h_{n}$ are computed using Eq. (2.53), and the coefficients $\eta_{n}$ are given by $\frac{k_{z_{n}}^{A}}{\omega \epsilon_{0}}$

In the cavity region, the tangential magnetic field is represented as

$$
H^{B}=-\mathrm{j} \omega \hat{\epsilon} \sum_{m=1}^{M} \sum_{n=0}^{N-1} \frac{V_{n} C_{m n}}{k_{z_{m}}^{B}} J_{1}\left(k_{\rho_{m}}^{B} \rho\right) \cos \left(k_{z_{m}}^{B}(z-d)\right)
$$


where the coefficients $V_{n}$ are the magnetic current expansion coefficients and the constants $C_{m n}, \quad k_{\rho_{m}}^{B}$ and $k_{z_{n}}^{B}$ are given by Eqs. (2.60), (2.43), and (2.44), respectively.

The magnetic field tangential to the aperture using the two forms of Eqs. (2.61) and (2.62), at $1 \mathrm{GHz}$ frequency, for dielectric constant value of 3.0 , dielectric loss factor of 0.001 , thickness $d$ of $0.01 \mathrm{~cm}$, and cavity radius $c$ of $0.7458 \mathrm{~cm}$, was plotted. Excellent agreement in the aperture was obtained as seen in Figs. 2.3 and 2.4 showing the real and imaginary parts, respectively, of the aperture magnetic field.

\subsubsection{Aperture Electric Field}

As the aperture plane is approached, the electric field should satisfy: 1) $E_{\rho}(\rho)$ should approach infinity as $\rho$ approaches $a$ and $b$ and 2) $E_{\rho}(\rho)$ should be identically zero on the conductor for $0<\rho<a$ and $b<\rho<c$. The electric field tangential to the aperture plane from the transmission line analysis was plotted at $1 \mathrm{GHz}$ frequency, for dielectric constant value of 3.0, dielectric loss factor of 0.001 , dielectric thickness $d$ of $0.01 \mathrm{~cm}$, and cavity radius $c$ of $0.7458 \mathrm{~cm}$. From the cavity region analysis, the tangential electric field at the aperture plane, given by a Green's function representation of the aperture magnetic current source, was also plotted under the same specifications.

The real and imaginary parts for both fields agree and satisfy the criteria for the electric field, as shown in Figs. 2.5 and 2.6. 


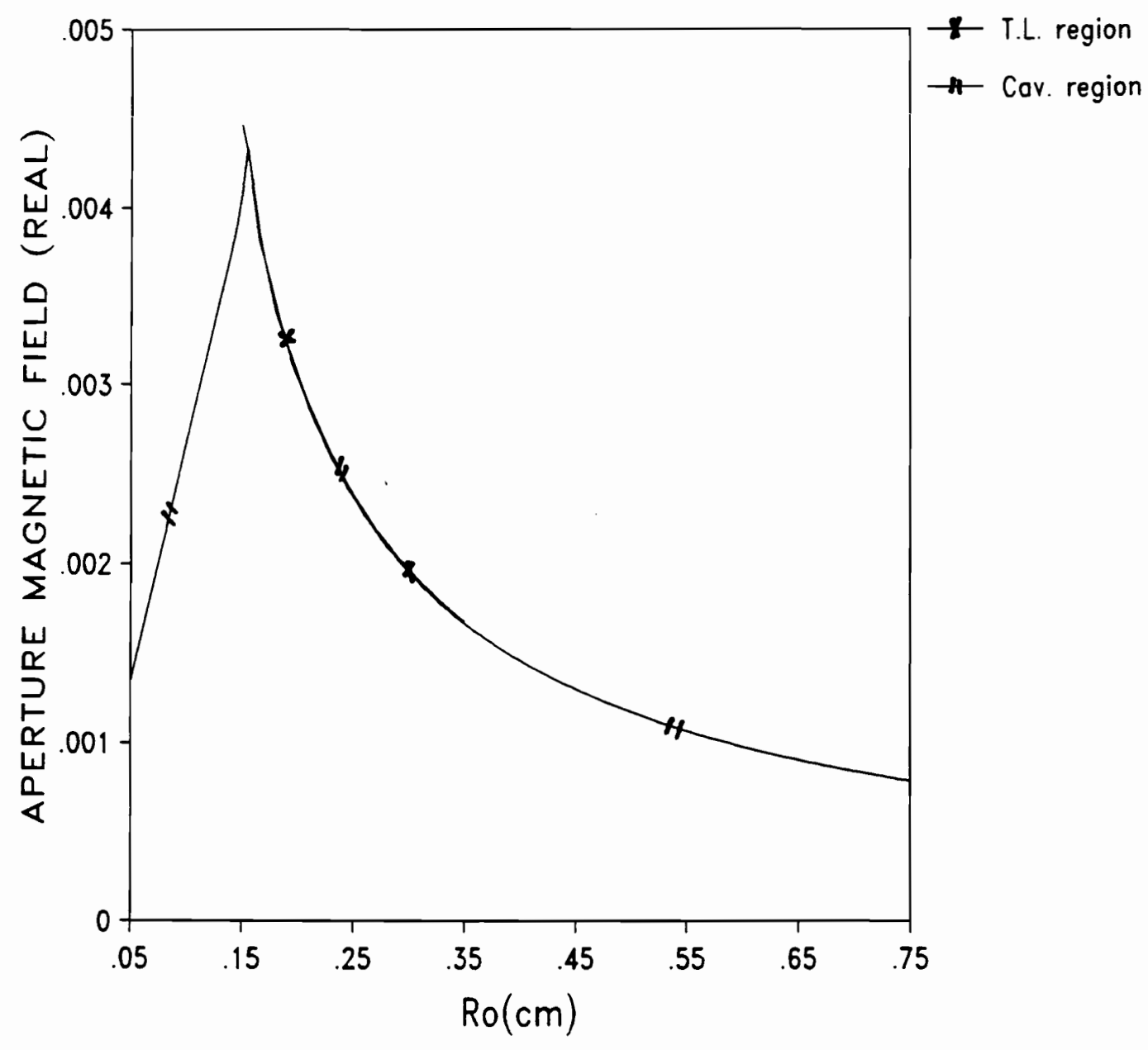

Figure 2.3. Aperture tangential magnetic field (real) calculated from both the transmission line region and the cavity region analysis, for a dielectric thickness of $0.01 \mathrm{~cm}, \epsilon^{\prime}=3$ and $\epsilon^{\prime \prime}=.001$ at $1 \mathrm{GHz}$ frequency. Both plots coincide in the aperture for $0.15 \mathrm{~cm} \geq \rho \leq 0.35 \mathrm{~cm}$. 


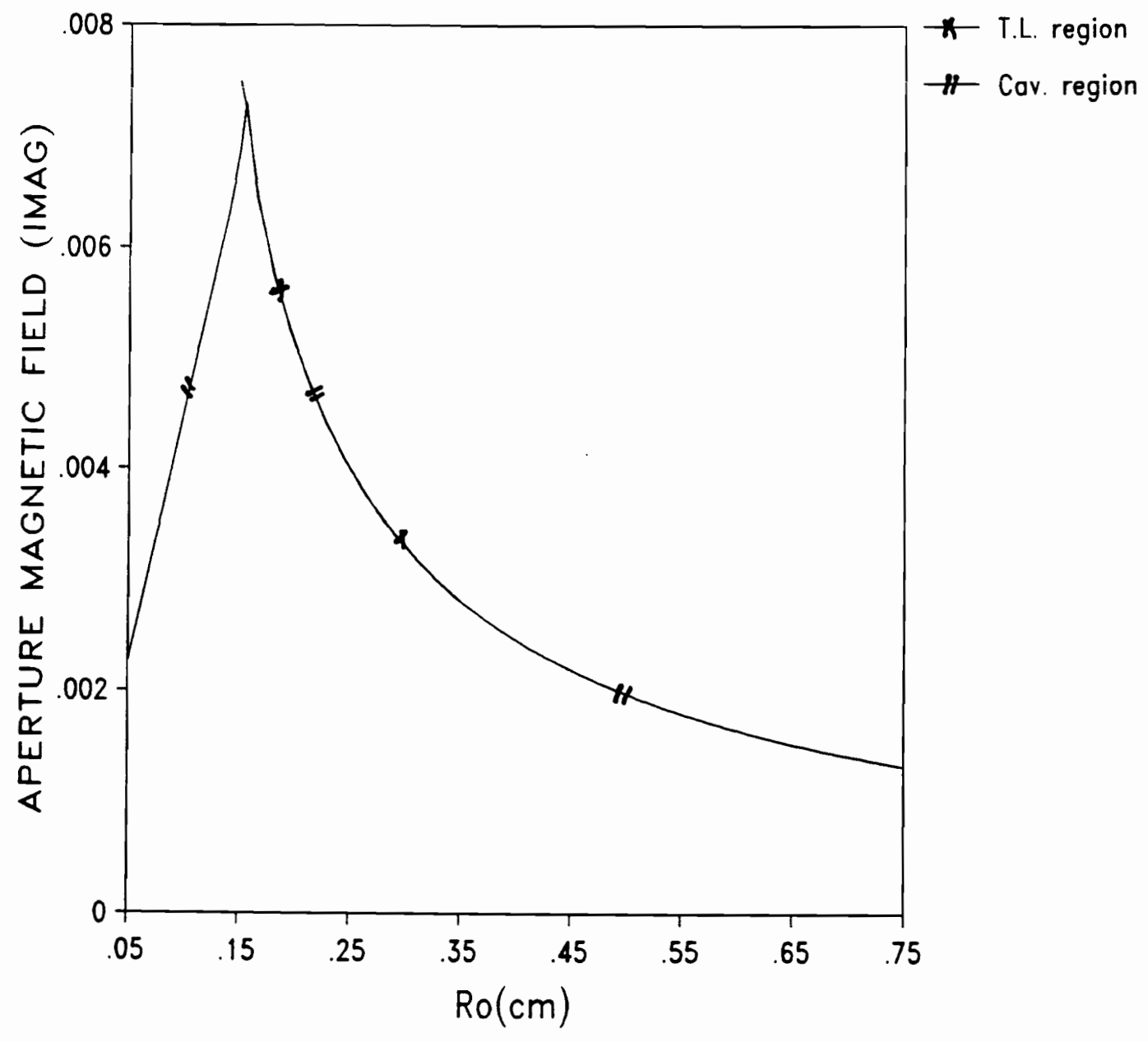

Figure 2.4. Aperture tangential magnetic field (imaginary) calculated from both the transmission line region and the cavity region analysis, for a dielectric thickness of $.01 \mathrm{~cm}, \epsilon^{\prime}=3$ and $\epsilon^{\prime \prime}=.001$ at $1 \mathrm{GHz}$ frequency. Both plots coincide in the aperture for $0.15 \mathrm{~cm} \geq \rho \leq 0.35 \mathrm{~cm}$. 


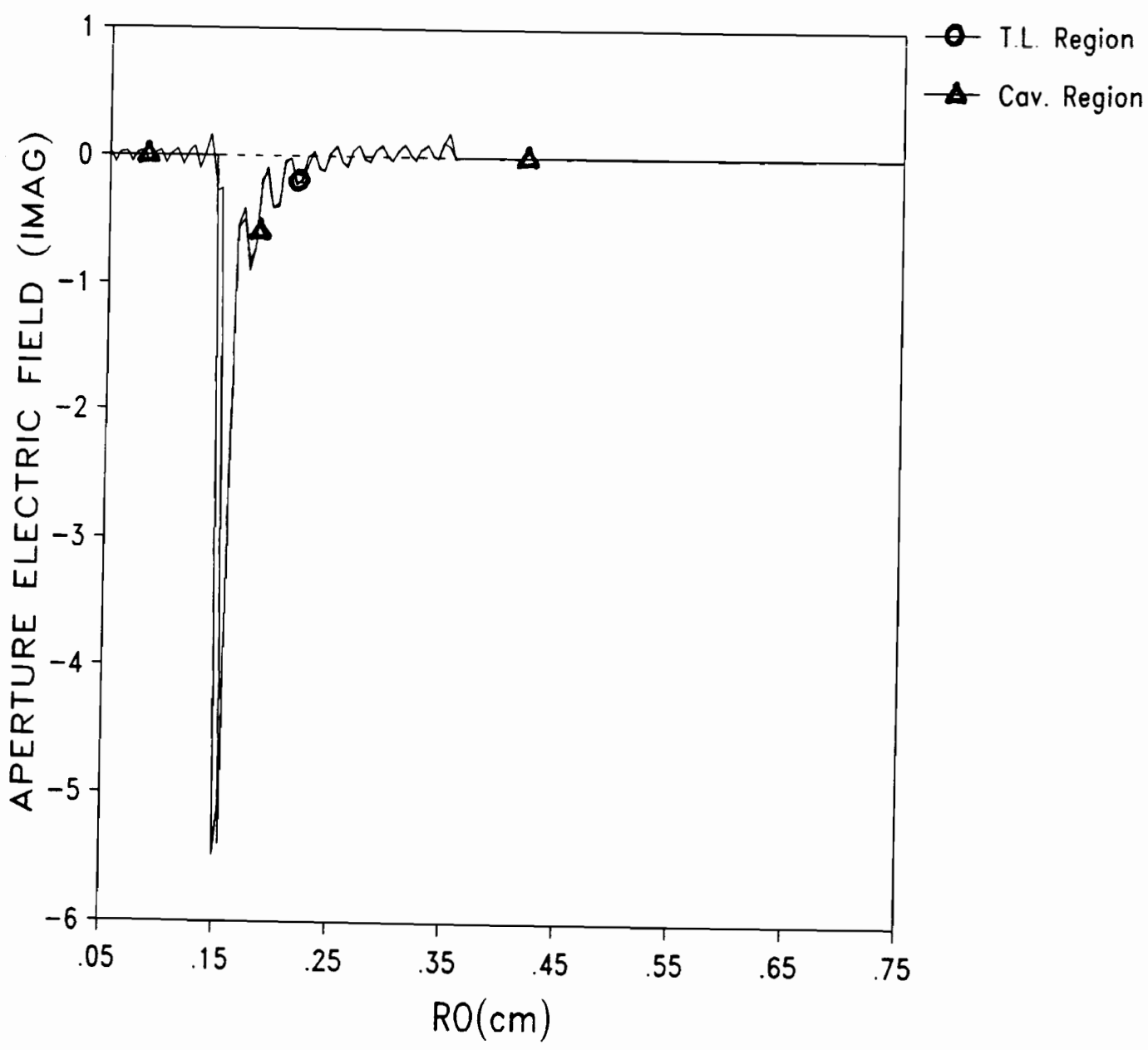

Figure 2.5. Aperture tangential electric field (real) calculated from both the transmission line region and the cavity region analysis, for a dielectric thickness of $0.1 \mathrm{~cm}, \epsilon^{\prime}=3, \epsilon^{\prime \prime}=.001$, and at $1 \mathrm{GHz}$ frequency. Both plots coincide in the aperture for $0.15 \mathrm{~cm} \geq \rho \leq 0.35 \mathrm{~cm}$. 


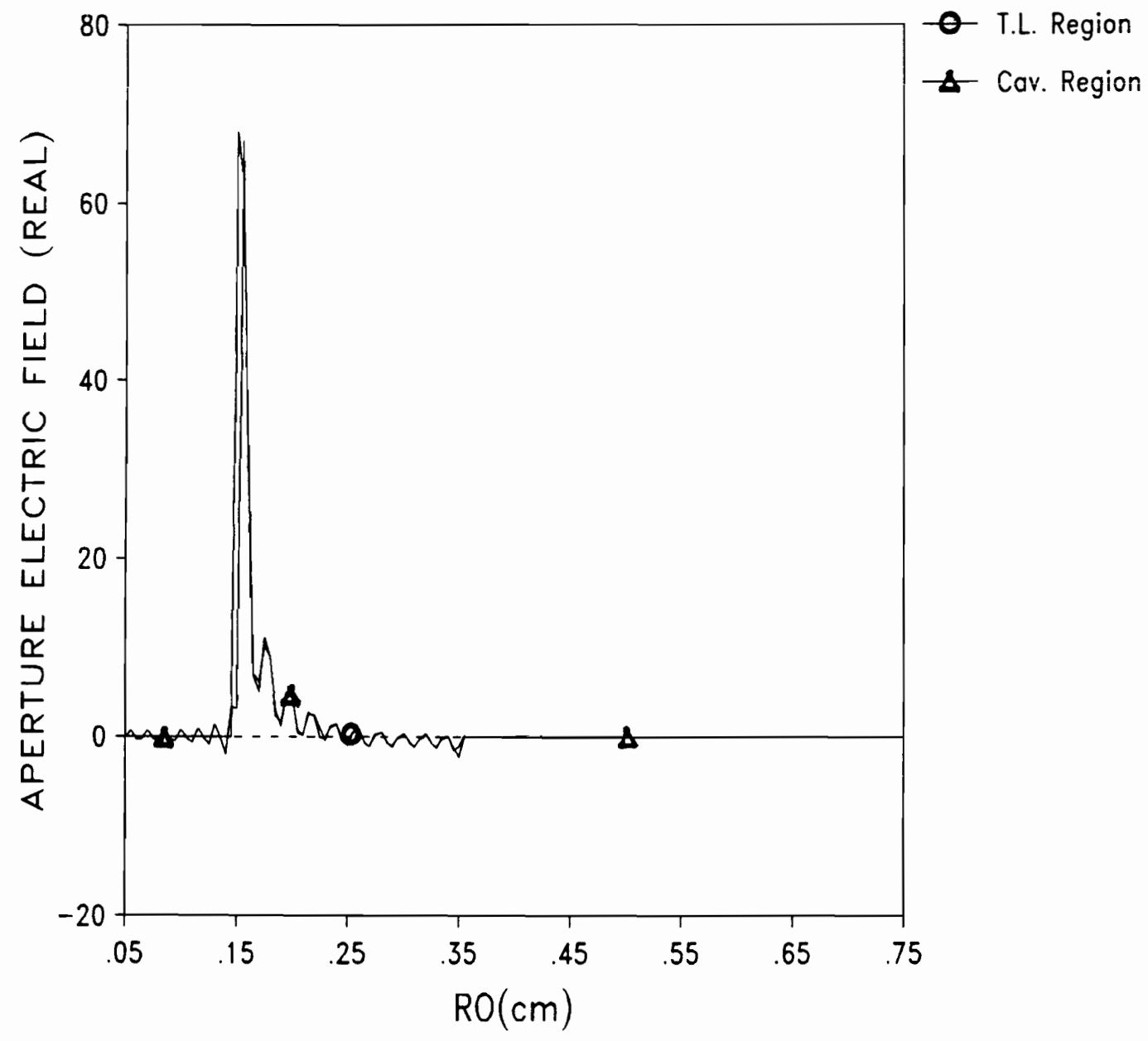

Figure 2.6. Aperture tangential electric field (imaginary) calculated from both the transmission line region and the cavity region analysis, for a dielectric thickness of $0.1 \mathrm{~cm}, \epsilon^{\prime}=3$, $\epsilon^{\prime \prime}=.001$, and at $1 \mathrm{GHz}$ frequency. Both plots coincide in the aperture for $0.15 \mathrm{~cm} \geq \rho \leq 0.35 \mathrm{~cm}$. 


\subsubsection{Number of Modes Determination}

The relationship of truncation of the number of modes $N$ in the transmission line region $A$ and the number of terms used in the Green's function expansion $M$ in the cavity region $B$ is now considered. The effect of this truncation on the reflection coefficient phase, affecting the determination of the dielectric constant, is also studied in this work. The reference phase was computed using a large number of modes in both regions $(N=100, M=800)$. The reflection coefficient phase was then determined versus the number of modes in the cavity region while stepping over different numbers of modes in the transmission line region $(N=1,10,20$, and 50). These relations are plotted at different frequencies, dielectric thicknesses, and transmission line and cavity dimensions as shown in Figs. 2.7, 2.8, 2.9, and 2.10, respectively. The study shows that for any number of modes $N$ in the transmission line region, the solution converges with respect to the number of modes in the cavity region for $M$ greater than a specific value dependent on $N$ and the geometry of the two structures. The relation is suggestive of the $\frac{M}{N}$ ratio for similar asymptotic convergence [Mittra \& Lee, 1971] in bifurcated-waveguide and step-type discontinuity problems and was found to be

$$
M=\left(\frac{c}{b-a}\right) N
$$

where $c$ is the cavity radius and $a$ and $b$ are the transmission line inner an outer radii, respectively. The convergence point was determined by computing the relative error in phase with respect to the preceding value.

For the different values of $N$ taken in the study, with $M$ determined by Eq. (2.63), convergence was obtained but not necessarily to the reference phase. 


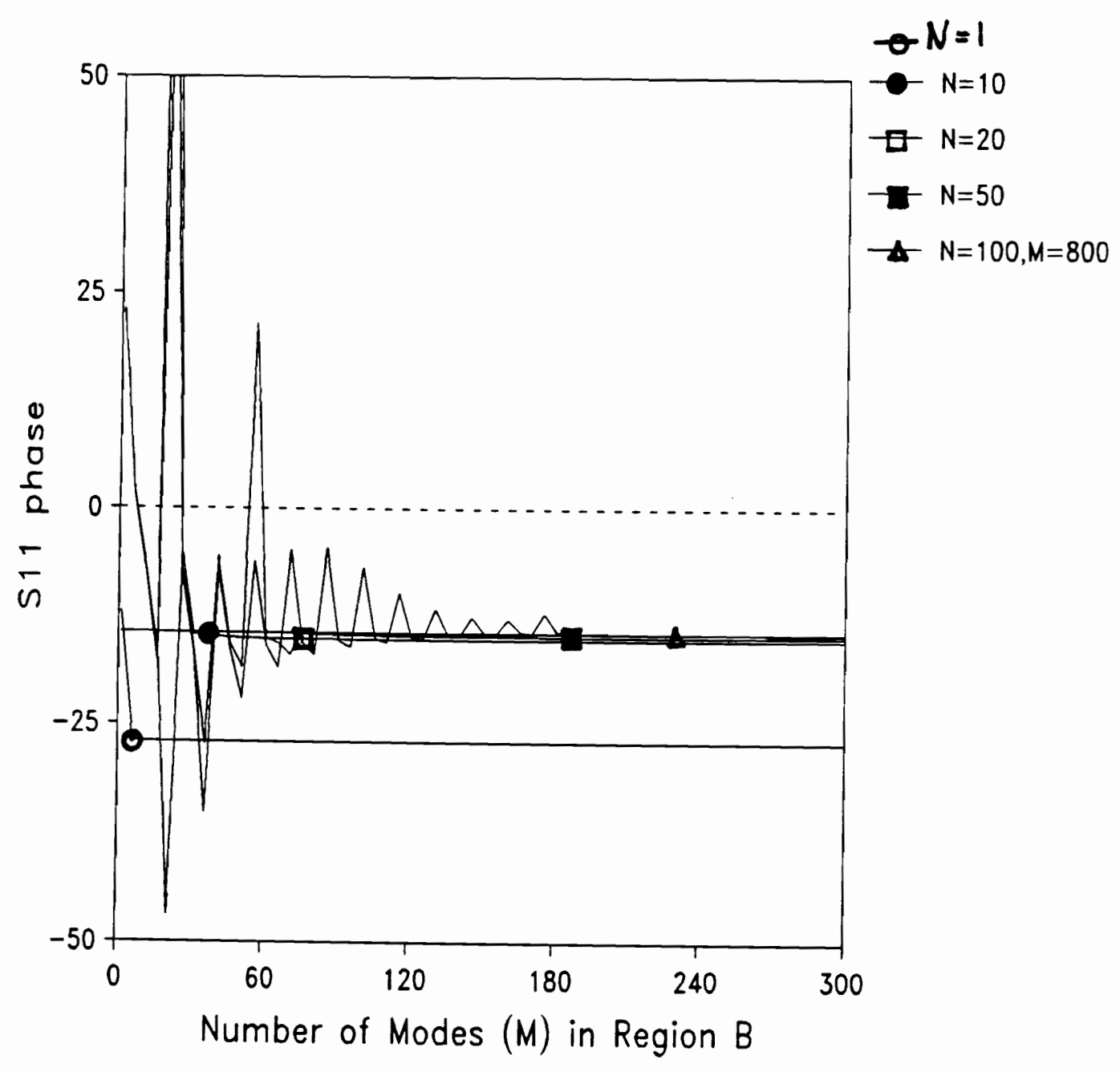

Figure 2.7. Effect of modes truncation on solution convergence. Plots of $\mathrm{s}_{11}$ phase versus $M$ with different values of $N$, for a dielectric thickness of $0.05 \mathrm{~cm}, \epsilon^{\prime}=3, \epsilon^{\prime \prime}=.001$, and at $1 \mathrm{GHz}$ frequency. The markers denote the points of convergence. 


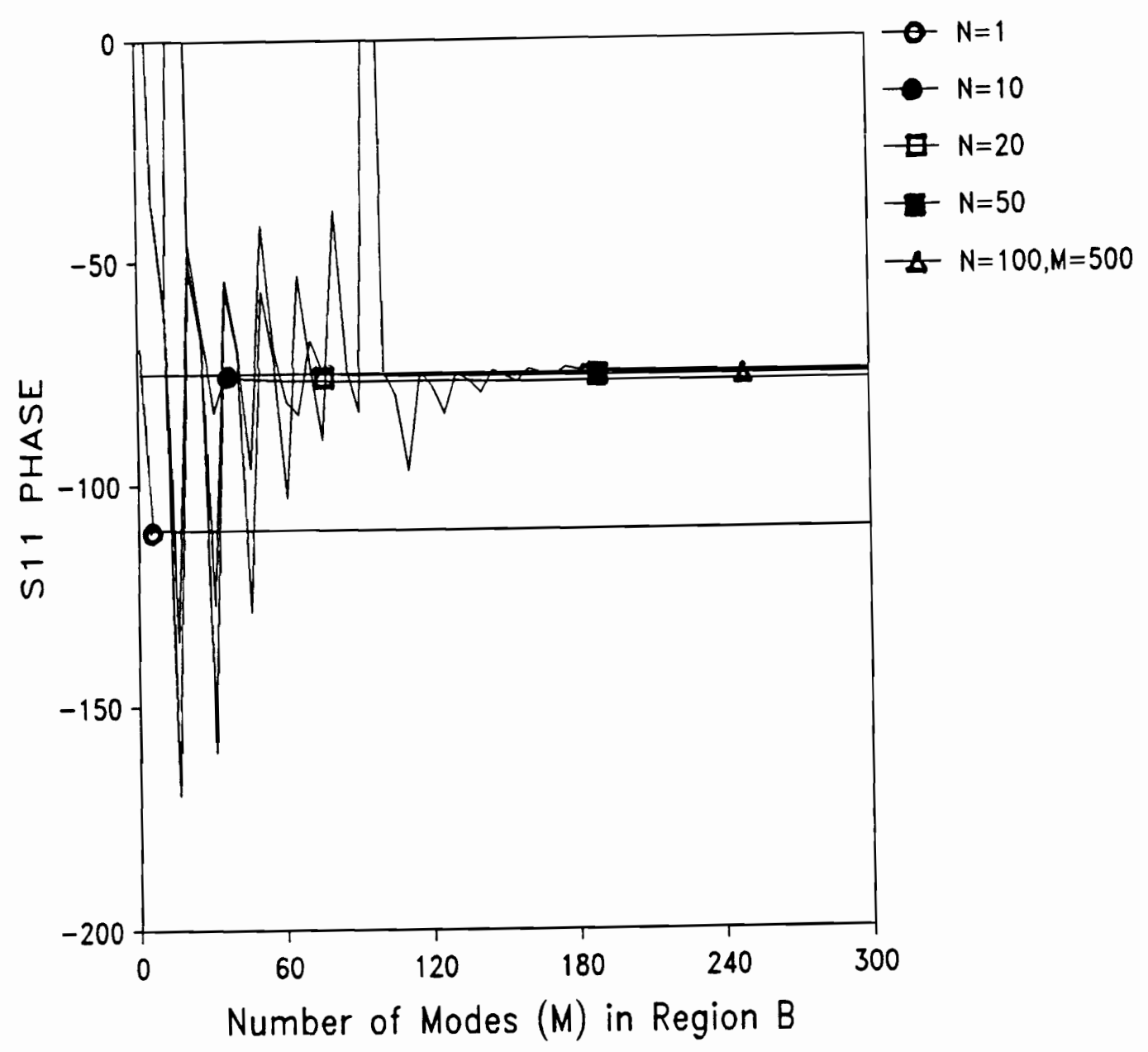

Figure 2.8. Effect of modes truncation on solution convergence. Plots of $\mathrm{s}_{11}$ phase versus $M$ with different values of $N$, for a dielectric thickness of $0.05 \mathrm{~cm}, \epsilon^{\prime}=3, \epsilon^{\prime \prime}=.001$, and at $4 \mathrm{GHz}$ frequency. The markers denote the points of convergence. 


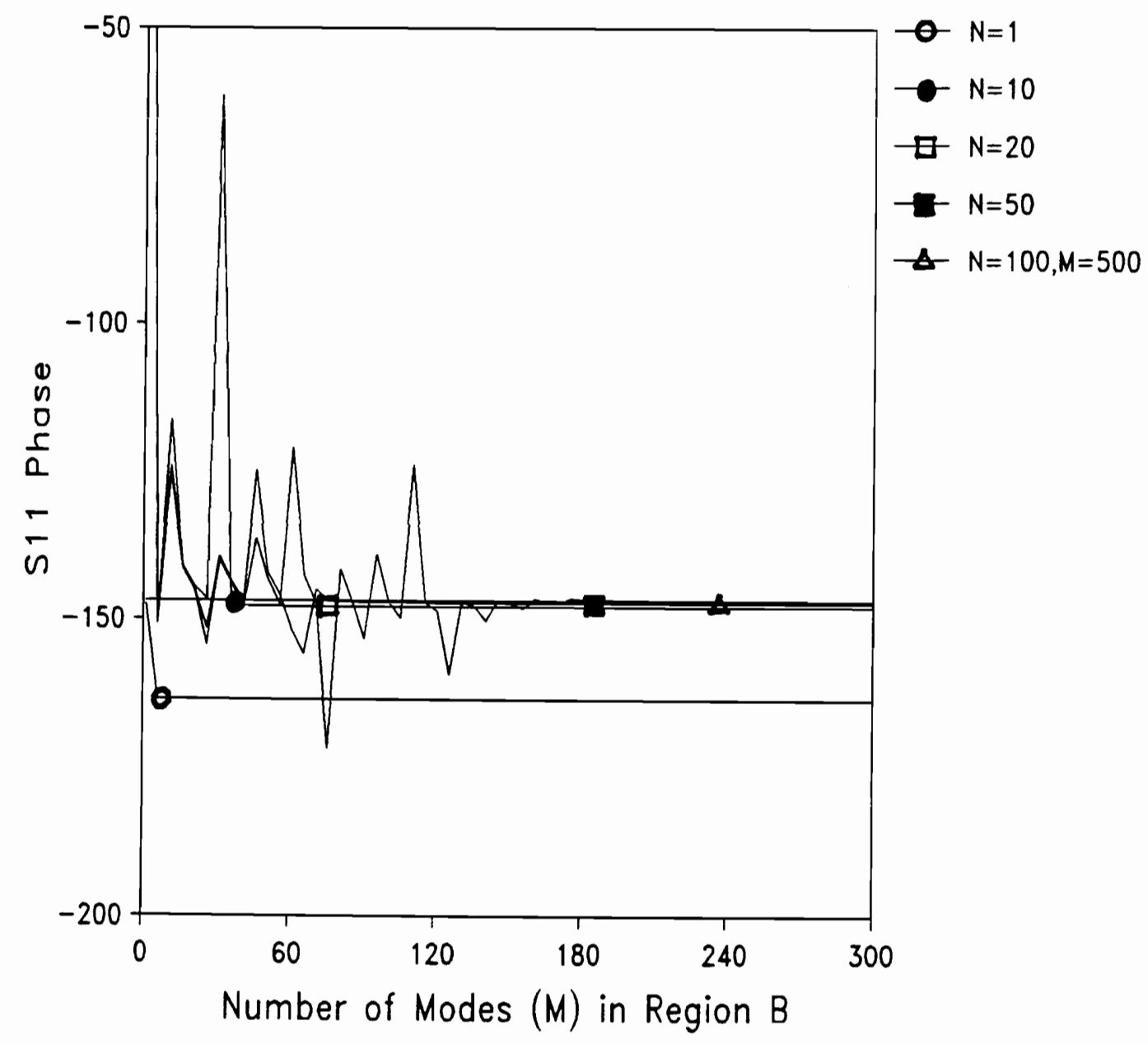

Figure 2.9. Effect of modes truncation on solution convergence. Plots of $\mathrm{s}_{11}$ phase versus $M$ with different values of $N$, for a dielectric thickness of $0.01 \mathrm{~cm}, \epsilon^{\prime}=3, \epsilon^{\prime \prime}=.001$, and at $4 \mathrm{GHz}$ frequency. The markers denote the points of convergence. 


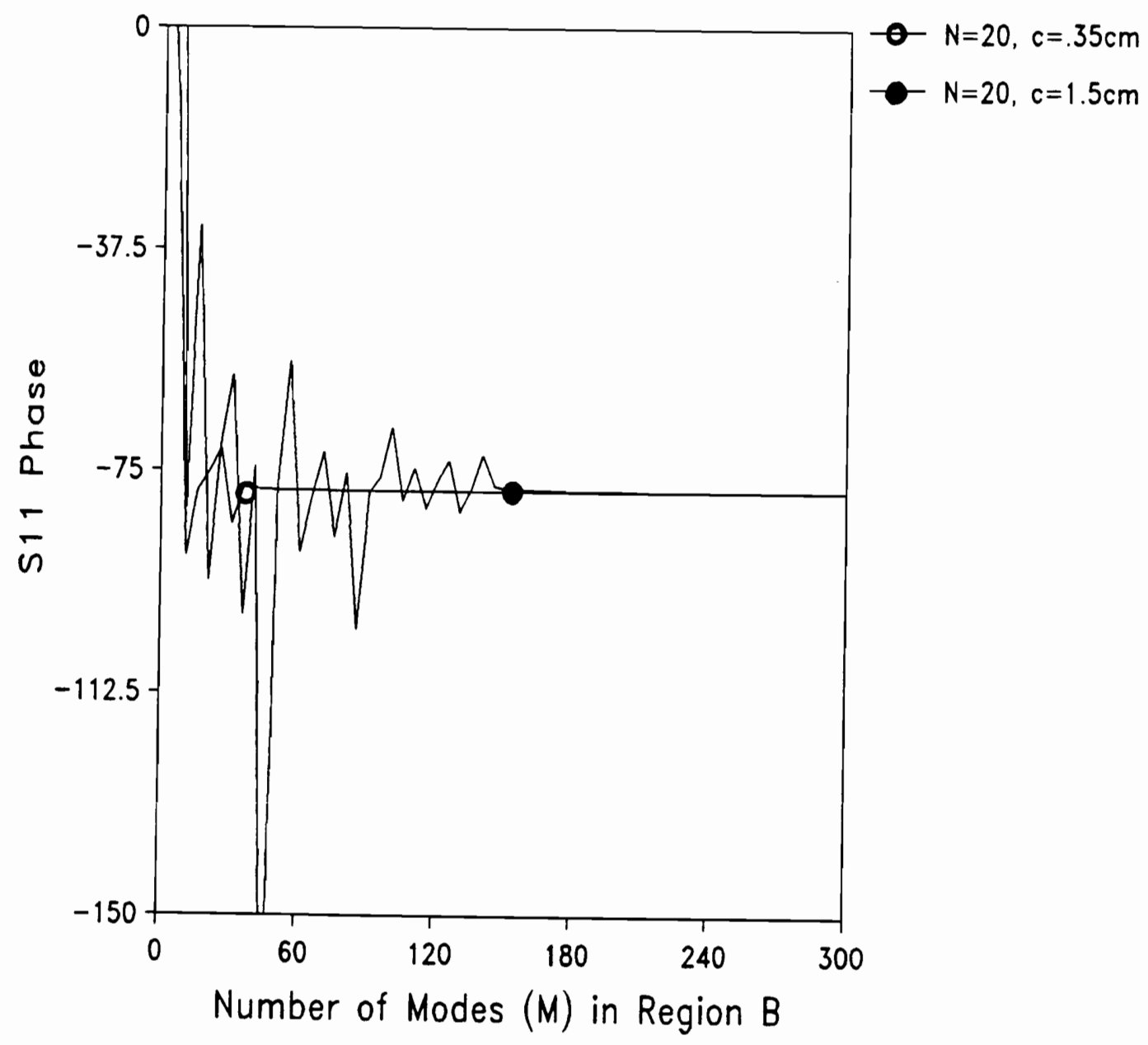

Figure 2.10. Effect of modes truncation on solution convergence. Plots of $\mathrm{s}_{11}$ phase versus $M$ for $N$ equals 20 , a dielectric thickness of $0.05 \mathrm{~cm}, \epsilon^{\prime}=3, \epsilon^{\prime \prime}=.001,1 \mathrm{GHz}$ frequency and different cavity radii $c$ of $0.35 \mathrm{~cm}$ and $1.5 \mathrm{~cm}$. The markers denote the points of convergence. 
Comparing the convergence values for the different $N$, it was found that the relative error defined as

$$
\text { Relative Error }(\%)=\frac{\text { (convergence value }- \text { reference value })}{\text { reference value }} \times 100
$$

decreases monotonically upon increasing $N$. For example, for $N$ equals twenty there was a $0.1 \%$ relative error, while for $N$ equals fifty the relative error is $0.03 \%$. Thus a minimum of twenty will be used to obtain a reasonable convergence relative to the reference point.

\subsection{SUMMARY}

The unperturbed problem considered in this chapter was a reflection cavity structure designed to adapt to the $7 \mathrm{~mm}$ connector of the HP $8510 \mathrm{~B}$ network analyzer. The cavity problem was modeled as two guided wave regions connected through an aperture. The aperture separating the two regions of the structure is modeled by equivalent magnetic current sources. The magnetic surface currents are expanded in terms of the transmission line modes and the resultant magnetic fields are matched at the aperture.

This chapter developed the solution of the unperturbed problem, parallel to the work of Saed [1987], using a more general approach. A basic Green's function solution approach was developed and then applied to the cavity structure by expanding the Green's functions in both regions in terms of the modes of the structures respectively. The series in the Green's functions expansions were truncated and field continuity was applied over the aperture. The magnetic 
current sources imposed on the aperture may be solved for, using the method of moments.

The accuracy of the solution due to higher order modes, was checked by comparing the fields in both the transmission line and the cavity regions and getting excellent match at the aperture. The relation between the number of modes in the transmission line region and the number of modes in the cavity region was investigated in this chapter to ensure solution convergence. The minimum number of modes retained in the series, in order to obtain a reasonable convergence to a reference obtained using very large numbers of modes, was also studied. 


\section{CHAPTER 3}

\section{THE PERTURBATION PROBLEM}

\subsection{INTRODUCTION}

Perturbation is a technique used to approximately solve a problem that closely resembles one that is exactly solvable. Even for problems which can be solved exactly, it may be more convenient to employ this approximate method, for the evaluation of the exact solution may be much too complicated. The perturbation technique evaluates the change in a quantity due to a small change in the problem. The word "perturb" means to disturb or to change slightly. An example of an unperturbed problem, for which solution is known, is a cavity resonator formed by a perfect conductor enclosing a volume. The perturbed problem could then be one with a slightly different surface covering a slightly different volume, one with a different material filling the cavity, or one with an imperfectly conducting surface. The quantity to be examined might be the resonant frequency or the unloaded $Q$ factor. Another unperturbed problem is a loss-free waveguide with perfectly conducting walls. The perturbed problem may involve a wall perturbation, a material perturbation, or imperfectly conducting walls. The parameter to be evaluated might be the cutoff frequency, the characteristic impedance, or the attenuation constant.

This chapter develops first, as an example, a perturbation approach to solve for the attenuation introduced in a hollow rectangular waveguide due to the losses in its imperfectly conducting walls. A perturbation approach is then applied to the unperturbed problem introduced in Chapter 2 to estimate the dielectric loss 
tangent by taking into account the conductor loss due to imperfectly conducting cavity walls. The technique computes the total loss from the measured reflection coefficient and the conductor loss using a perturbation approach. The power loss in the cavity walls is then subtracted from the total loss to give the power loss in the dielectric from which a better estimate of the dielectric loss tangent is obtained.

\subsection{A CANONICAL PERTURBATION EXAMPLE}

To examine closely the perturbation approach we consider the example of a hollow rectangular waveguide with perfect conductor walls. The guide has lateral dimensions $a$ and $b$ as shown in Fig. 3.1. It is assumed to be of infinite length, and empty. The dominant mode, in this case the $\mathrm{TE}_{10}$ traveling in the $z$ direction, will be considered. The time harmonic electric and magnetic fields must satisfy Maxwell's equations for a source-free region. Assuming an $e^{j \omega t}$ time dependence we have

$$
\begin{aligned}
& \nabla \times \bar{E}=-\mathrm{j} \omega \mu_{0} \overline{\boldsymbol{H}} \\
& \nabla \times \overline{\boldsymbol{H}}=\mathrm{j} \omega \epsilon_{0} \overline{\boldsymbol{E}}, \\
& \nabla \cdot \overline{\boldsymbol{E}}=0, \\
& \nabla \cdot \overline{\boldsymbol{H}}=0 .
\end{aligned}
$$

Working with the above equations and eliminating $\bar{E}$, we obtain the Helmholtz wave equation

$$
\nabla^{2} \overline{\boldsymbol{H}}+k^{2} \overline{\boldsymbol{H}}=0
$$




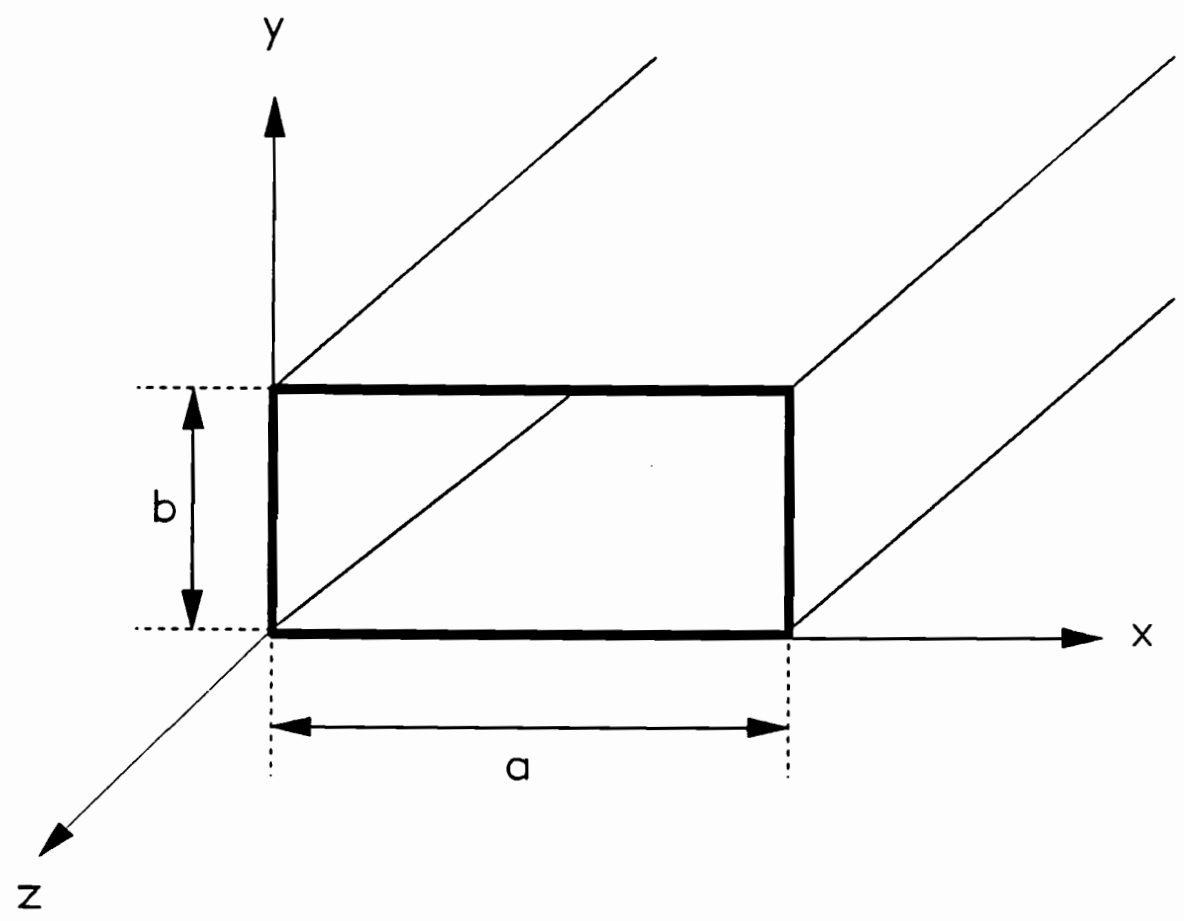

Figure 3.1. Rectangular waveguide with its appropriate dimensions. 
where $k^{2}=\omega^{2} \epsilon_{0} \mu_{0}, \epsilon_{0}$ is the free-space permittivity, and $\mu_{0}$ is the free-space permeability.

For this waveguide structure, the necessary and sufficient boundary conditions are that the tangential components of the electric field must vanish on the guide walls. Using the separation of variables method, we solve for the fields to obtain the following electric and magnetic field components for the $\mathrm{TE}_{10}$ mode:

$$
\begin{aligned}
& E_{x}(x, y, z)=E_{z}(x, y, z)=H_{y}(x, y, z)=0 \\
& E_{y}(x, y, z)=-H_{0} \frac{\mathrm{j} \omega \mu_{0}}{k_{c}^{2}}\left(\frac{\pi}{a}\right) \sin (\pi x / a) e^{-\mathrm{j} k_{z} z}, \\
& H_{x}(x, y, z)=H_{0} \frac{\mathrm{j} k_{z}}{k_{c}^{2}}\left(\frac{\pi}{a}\right) \sin (\pi x / a) e^{-\mathrm{j} k_{z} z}, \\
& H_{z}(x, y, z)=H_{0} \cos (\pi x / a) e^{-\mathrm{j} k_{z} z} .
\end{aligned}
$$

Here

$$
k_{c}^{2}=(\pi / \mathrm{a})^{2},
$$

and

$$
k_{z}^{2}=\omega^{2} \epsilon_{0} \mu_{0}-k_{c}^{2}
$$

The waveguide supports only an attenuating wave for the frequency below the cutoff frequency $f_{c}$ of the guide given by

$$
f_{c}=\frac{1}{2 \mathrm{a} \sqrt{\epsilon_{0} \mu_{0}}}
$$


which gives $k_{z}^{2}$ as

$$
k_{z}^{2}=\omega^{2} \epsilon_{0} \mu_{0}\left[1-\left(f_{c} / f\right)^{2}\right]
$$

After solving for the fields in an exact way, we may perturb the problem to include attenuation in the waveguide due to imperfectly conducting walls. There will be a continuous loss of power in the walls as the mode propagates along the guide. The propagation constant $\mathrm{j} k_{z}$ is perturbed and becomes $\mathrm{j} k_{z_{p}}=\mathrm{j} k_{z}+\alpha$ [Collin, 1966], where $\alpha$ is an attenuation constant that gives the rate at which the mode amplitude must decay as the mode propagates along the guide. To solve exactly for the loss associated with this change, it would mean solving the new boundary-value problem with more complicated boundary conditions at the walls. This creates a complicated field-matching problem at the conduction interface. If the walls have a high conductivity, as for good conductors with conductivity on the order of $10^{7}$ to $10^{8} \mathrm{~S} / \mathrm{m}$, we may instead assume that the electric and magnetic fields in the guide are only slightly perturbed from those with the perfectly conducting walls and the fields are essentially the same. In other words, the cross sectional distribution of the fields is essentially unchanged except about nulls. The loss in the guide will be responsible for an attenuation constant $\alpha_{c}$ which accounts for the power losses in the conductor walls. Using $\alpha_{c}$ to describe the loss is based on an exponential power decay of the form

$$
P_{f}=P_{0} \mathrm{e}^{-2 \alpha_{c} z}
$$

where $P_{f}$ is the time-average power flow traveling in the waveguide, and $P_{0}$ is the power at some reference point (i.e., $z=0$ ), as shown in Fig. 3.2. The power loss 


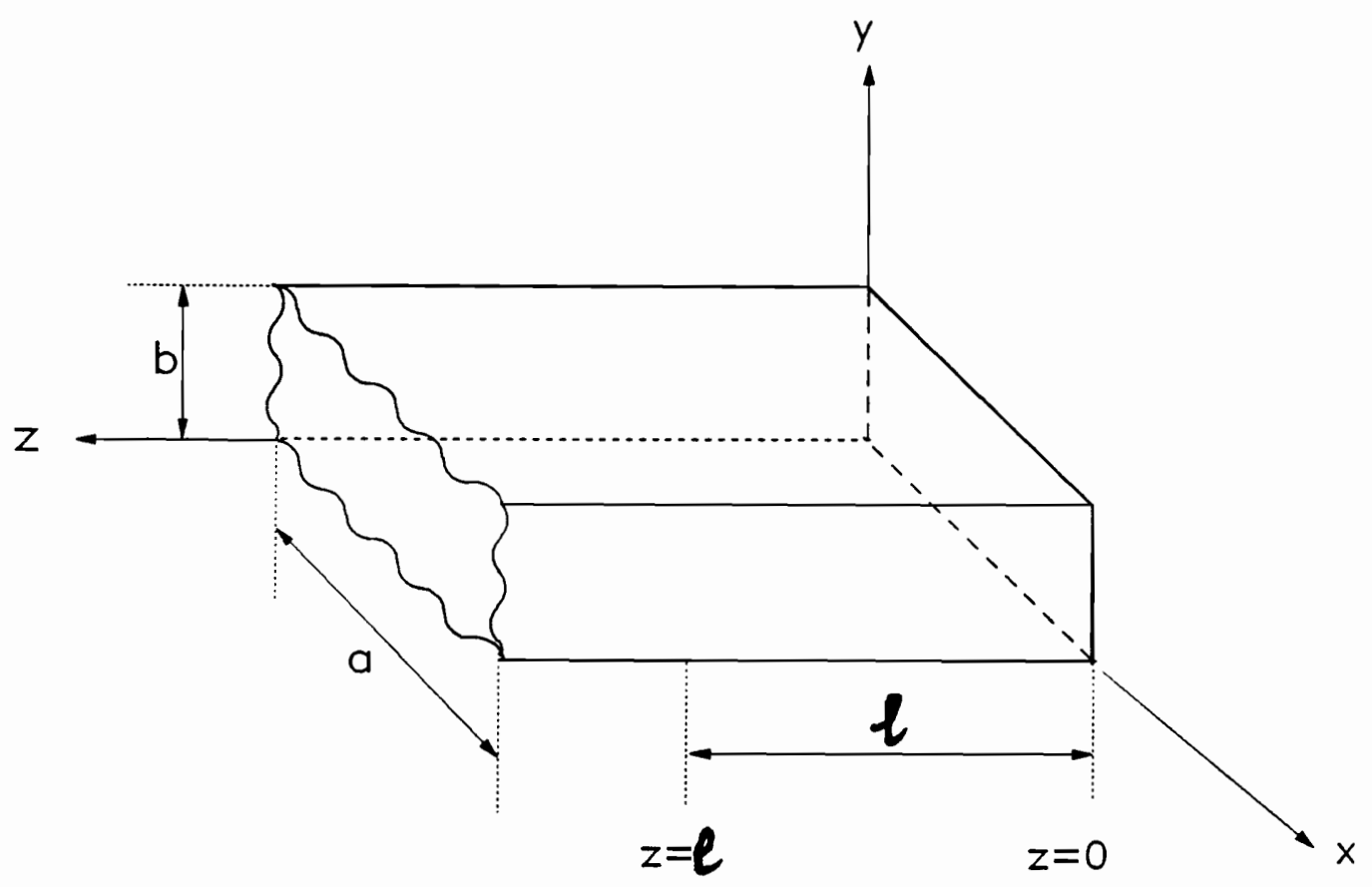

Figure 3.2. Power flow along a waveguide with an attenuation constant $\alpha_{c}$. 
per unit length in the conductor may be equated to the negative rate of change of $P_{f}$ versus $z$ given by the expression

$$
P_{d} / \ell=2 \alpha_{c} P_{f}
$$

Thus, the attenuation constant due to losses in the imperfectly conducting walls may be represented as

$$
\alpha_{c}=\frac{P_{d} / \ell}{2 P_{f}}
$$

where $P_{d} / \ell$ is the time-average dissipated power per unit length on all four walls of the waveguide, and $P_{f}$ is the time-average power flow traveling in the waveguide.

The total power traveling in the guide is obtained by integrating the $z$ directed time-averaged power density, or Poynting vector, over a cross section $A$ of the guide.

$$
P_{f}=\int_{A} \operatorname{Re}(\bar{S}) \cdot \hat{z} d s
$$

The z-directed Poynting vector $S_{z}$ is defined as

$$
S_{z}=\frac{1}{2}\left(\bar{E} \times \bar{H}^{*}\right) \cdot \hat{z}=\frac{1}{2}\left(E_{x} H_{y}^{*}-E_{y} H_{x}^{*}\right),
$$

and the cross section of the rectangular waveguide is the rectangular surface with 
dimensions $a$ and $b$. The resultant power flow in the guide for the $\mathrm{TE}_{10}$ mode is

$$
P_{f}=\int_{0}^{b} \int_{0}^{a} \frac{1}{2} \frac{\omega \mu_{o} \beta_{z}\left|H_{o}\right|^{2}}{k_{c}^{4}}\left(\frac{\pi}{a}\right)^{2} \sin ^{2}\left(\frac{\pi x}{a}\right) e^{-2 \alpha_{c} z} d x d y
$$

or

$$
P_{f}=\omega \mu_{o} \beta_{z}\left|H_{o}\right|^{2}\left(\frac{\mathrm{a}}{\pi}\right)^{2} \frac{a b}{4} e^{-2 \alpha_{c} z}
$$

where $\beta_{z}$ is the $k_{z}$ of the unperturbed lossless problem given by Eq. (3.5).

The perturbed problem has imperfectly conducting walls with assumed plane waves penetrating the conductor surface, but localized in a thin surface layer. The thickness of this layer is on the order of $\delta=(2 / \omega \mu \sigma)^{1 / 2}$. This phenomenon is known as the skin effect. The electric field will have a tangential component at the walls $\bar{E}_{t a n g}$, where the original problem has a null, thus the key to the solution is to use the magnetic field which is near a maximum. The propagation constant of the waves in the conductor will have components both tangential and perpendicular to the dielectric-conductor interface such that

$$
\bar{E}=\bar{E}_{0} e^{-\gamma_{t} \hat{\boldsymbol{t}}-\gamma_{p} \hat{\boldsymbol{p}}}
$$

with $\hat{\boldsymbol{t}}$ and $\hat{\boldsymbol{p}}$ denoting tangential and perpendicular unit vectors respectively. This satisfies the homogeneous Helmholtz equation, provided that

$$
\gamma_{c}^{2}=\gamma_{t}^{2}+\gamma_{p}^{2}
$$

where $\gamma_{c}$ is the propagation constant in the conductor region, $\gamma_{t}$ is the propagation 
constant in the tangential direction, and $\gamma_{p}$ is the propagation constant in the perpendicular direction. The propagation constant in the tangential direction is on the order of the propagation constant in the dielectric material given by $\mathrm{j} \sqrt{\omega^{2} \epsilon_{d} \mu_{0}}$, while the propagation constant in the conductor region, where the displacement current $\mathrm{j} \omega \epsilon \bar{E}$ can be neglected in comparison with the conduction current $\sigma \bar{E}$, is equal to $\sqrt{j \omega \mu_{0} \sigma}$. So the propagation constant in the perpendicular direction may be found as

$$
\gamma_{p} \simeq \sqrt{\mathrm{j} \omega \mu_{0} \sigma+\omega^{2} \epsilon_{d} \mu_{0}} \simeq \sqrt{\mathrm{j} \omega \mu_{0} \sigma}=\gamma_{c}
$$

So the plane waves propagating in the conductor are mainly propagating in the perpendicular $\hat{\boldsymbol{p}}$ direction, and may be represented by

$$
\overline{\boldsymbol{E}}=\overline{E_{0}} e^{-\gamma_{c} \hat{\boldsymbol{p}}}
$$

This derivation is under the assumption of neglecting the reflected waves from the back of the conductor walls since it will be proved that for thick enough conductor walls, the penetrating waves will be localized in a thin surface layer independent of the thickness of the conductor. The normal-directed power density in the conductor walls may be evaluated as

$$
S_{p}=\frac{\sigma}{2} \int_{0}^{d}|\bar{E}|^{2} d p=\frac{\sigma}{2}\left|\bar{E}_{0}\right|^{2} \frac{1}{2 \alpha_{c}}\left(1-\mathrm{e}^{-2 \alpha_{c} d}\right),
$$

where $d$ is the thickness of the conductor walls, $\alpha_{c}$ is the real part of the propagation constant $\gamma_{c}$, and $\sigma$ is the conductivity of the imperfectly conducting walls. Assuming that the thickness of the conductor walls is larger than half the skin depth $\gtrsim$ 
skin depth of the conductor defined by $\frac{1}{\alpha_{c}}$, such that $\mathrm{e}^{-2 \alpha_{c} d}$ is small enough to be neglected with respect to unity in Eq. (3.12), we obtain

$$
S_{p}=\frac{1}{2} \frac{\sigma}{2 \alpha_{c}}\left|\overline{E_{0}}\right|^{2}
$$

Using the relation between the electric and magnetic fields for plane waves given by

$$
\bar{E}=-\left(\hat{p} \eta_{c} \times \bar{H}\right)=\eta_{c}(\hat{\boldsymbol{n}} \times \overline{\boldsymbol{H}})
$$

where $\eta_{c}$ is the intrinsic impedance of the conductor material as $\sqrt{\mathrm{j} \omega \mu_{0} / \sigma}$, and $\hat{\boldsymbol{n}}=-\hat{\boldsymbol{p}}$ is the outward vector normal to the surface of the walls. Substituting with Eq.(3.14) for $\overline{E_{0}}$ in Eq.(3.13) with the value of $\alpha_{c}=\sqrt{\omega \mu_{0} \sigma / 2}$, we obtain

$$
S_{p}=\frac{1}{2} \sqrt{\frac{\omega \mu_{0}}{2 \sigma}}\left|\left(\hat{n} \times \bar{H}_{0}\right)\right|^{2}=\frac{1}{2} R_{s}\left|\bar{J}_{s}\right|^{2} .
$$

The surface current density $\bar{J}_{s}$ is given by $\hat{n} \times \overline{\boldsymbol{H}}_{0}$, where $\overline{\boldsymbol{H}}_{0}$ is the tangential component of the magnetic field to the walls. $\overline{\boldsymbol{H}}_{0}$ may be replaced with minimal error with the value corresponding to perfectly conducting walls. The quantity $R_{s}$ is the real part of $\eta_{c}$ and is defined as $\sqrt{\omega \mu_{0} / 2 \sigma}$. Observing the units of $R_{s}$, we realize that it has units of ohms, and will be defined as the surface resistance. The surface current density has units of $(\mathrm{A} / \mathrm{m})$, which gives the power density $S_{p}$ the corresponding units of Watts $/ \mathrm{m}^{2}$. The expression given in Eq. (3.15) is analogous to $I^{2} R / 2$ used in lumped circuit theory.

It is interesting to note that the intrinsic impedance of the conductor $\eta_{c}$ becomes a surface impedance $Z_{s}$ associated with the surface current density $\bar{J}_{s}$ and 
given by

$$
Z_{s}=R_{s}+\mathrm{j} X_{s} \simeq \sqrt{\frac{\omega \mu_{0}}{2 \sigma}}(1+\mathrm{j})
$$

The real part of this impedance has been defined as the surface resistance $R_{s}$ and the waves penetrating the conductor are localized in a thin surface layer. The thickness of this layer is on the order of $\delta=(2 / \omega \mu \sigma)^{1 / 2}$, and called the skin depth.

The time-averaged dissipated power in the imperfectly conducting walls is actually the power flowing into the walls, and is obtained by integrating the component of the power density normal to the surface, over the area of the conductor walls $A_{w}$, and may be written as

$$
P_{d}=\frac{R_{s}}{2} \int_{A_{w}}\left|\bar{H}_{t a n g}\right|^{2} d s .
$$

Eq. (3.17) has been derived for plane conductors, but we may use it for curved surfaces as long as the skin depth $(\delta)$ is much smaller than the significant radii of curvature of the conductor.

For the $\mathrm{TE}_{10}$ mode, the total power dissipated may be written as a sum of the power dissipated over the guide walls in a length $\ell$ as

$$
\begin{array}{r}
P_{d}=\frac{R_{s}}{2} 2\left\{\int_{0}^{\ell} \int_{0}^{a}\left|H_{x}\right|_{y=0}^{2} d x d z+\int_{0}^{\ell} \int_{0}^{a}\left|H_{z}\right|^{2}=0 d x d z\right. \\
\left.+\int_{0}^{\ell} \int_{0}^{b}\left|H_{z}\right|^{2}=0 d y d z\right\}
\end{array}
$$

where the factor of two accounts for twice the power dissipated on the bottom wall 
and twice the power dissipated in a side wall. Substituting the magnetic fields of the unperturbed problem in (3.18) and integrating, we obtain the power dissipated per unit length $\ell$ as

$$
P_{d} / \ell=R_{s}\left|H_{o}\right|^{2}\left\{\left(\frac{a}{2}\right)\left(1+k_{z}^{2}(a / \pi)^{2}\right)+b\right\} e^{-2 \alpha c^{2}},
$$

where

$$
k_{z}^{2}=\omega^{2} \epsilon_{0} \mu_{0}-(\pi / a)^{2} .
$$

Substituting the power dissipation per unit length given in Eq. (3.19) and the power flow given in Eq. (3.9) into Eq. (3.6) with the cutoff frequency given in Eq. (3.4) and $\beta_{z}$ as the $k_{z}$ given in Eq. (3.5), we obtain the attenuation in the guide due to the conductor walls for a frequency $f$ greater than the cutoff frequency $f_{c}$ as

$$
\alpha_{c}=\frac{R_{s}}{\eta \bar{b}} \sqrt{\frac{1}{1-\left(f_{c} / f\right)^{2}}}\left\{1+\frac{2 b}{a}\left(f_{c} / f\right)^{2}\right\} \quad(\mathrm{Np} / \mathrm{m})
$$

where $\eta$ is the intrinsic impedance of the dielectric filling the waveguide. In our case, the free-space intrinsic impedance is given by $\eta_{0}=\sqrt{\frac{\mu_{o}}{\epsilon_{o}}}$. This conduction loss agrees with the form given by Harrington [1961], who considered a $\mathrm{TE}_{01}$ mode.

\subsection{PERTURBATION OF THE CAVITY LOSS}

In cavity resonators, electromagnetic fields exist only at specific frequencies, called "resonant frequencies", in the loss-free region enclosed by a perfect conductor. When losses are present, a source must exist to sustain oscillations.

The technique considered uses a circular cavity structure, formed by a 
conductor enclosing a dielectric sample, and adapted to the end of a precision air line as illustrated in Fig. 2.1. The dielectric is assumed to be lossy and the cavity contains an aperture excited through the transmission line. This cavity structure is used over a wideband of frequencies away from resonance, which will allow the analysis of the problem to be done over a wide range of frequencies. Therefore this technique is given the name "Wideband Dielectric Filled Cavity", or WDFC for short.

The objective of this technique is to characterize low-loss dielectric materials by evaluating their complex permittivity $\hat{\epsilon}$. The complex permittivity $\hat{\epsilon}$, which is a function of frequency, is defined as

$$
\hat{\epsilon}=\epsilon_{0} \hat{\epsilon}_{r}=\epsilon_{0}\left(\epsilon_{r}^{\prime}-\mathrm{j} \epsilon_{r}^{\prime \prime}\right),
$$

where $\epsilon_{0}$ is the permittivity of free space, $\hat{\epsilon}_{r}$ is the relative complex permittivity, $\epsilon_{r}^{\prime}$ is the relative a-c capacitivity or dielectric constant, and $\epsilon_{r}^{\prime \prime}$ is the relative loss factor representing the dielectric loss.

The purpose of this technique is to establish a relationship between the complex permittivity of the dielectric $(\hat{\epsilon})$ and the reflection coefficient $\left(s_{11}\right)$ of the cavity structure, using a full-field analysis of the problem. The quantity $s_{11}$ (magnitude and phase) is measured using an HP 8510B network analyzer. The dielectric constant $\epsilon_{r}^{\prime}$ of the dielectric has been successfully evaluated, while the loss measured includes both the dielectric loss and that of the conductor walls. The objective of this dissertation is to separate the dielectric loss from the measured loss, using a perturbation approach. This method is based on the assumption that the introduction of a small loss in the conductor walls does not substantially 
perturb the fields from their loss-free values. The loss in the conductor walls is to be evaluated using the loss-free fields, and subtracted from the total loss in the unperturbed cavity which is calculated from the measured reflection coefficient, to estimate the dielectric loss. The unperturbed problem will be the problem of the cavity region filled with a low-loss dielectric, enclosed with perfectly conducting walls covering its surface except for an aperture coupling this cavity region to a transmission line region. The unperturbed field has no tangential electric field component at the conductor walls. The energy absorbed in the cavity is all stored and dissipated in the dielectric. The electric and magnetic fields in the cavity region will be represented by $\bar{E}$ and $\bar{H}$ respectively. The dielectric will be assumed to have a dielectric constant $\epsilon_{r a}^{\prime}$ and dielectric loss $\epsilon_{r a}^{\prime \prime}$, where the subscript $a$ stands for apparent values.

The perturbed problem will be the original problem "perturbed" to have imperfectly conducting walls. The solution for the fields is no longer exactly the same, because the boundary conditions are changed. In particular, the tangential component of the electric field at the walls is no longer zero. The electric and magnetic fields in the cavity region for this perturbed problem are represented by $\bar{E}_{p}$ and $\overline{\boldsymbol{H}}_{p}$, where the subscript $p$ stands for perturbed. The energy absorbed by the cavity is stored and dissipated in the dielectric as well as the conductor walls. The dielectric constant and loss of the perturbed cavity will be represented by $\epsilon_{r e}^{\prime}$ and $\epsilon_{r e}^{\prime \prime}$, where the subscript $e$ stands for estimated values.

In both problems, Maxwell's Equations must be satisfied as

$$
\nabla \times \bar{E}=-\mathrm{j} \omega \mu_{o} \overline{\boldsymbol{H}},
$$


and

$$
\nabla \times \bar{H}=\mathrm{j} \omega \hat{\epsilon} \bar{E}
$$

where $\mu_{o}$ is the permeability of free space for a nonmagnetic dielectric. Substituting with the complex permittivity $\hat{\epsilon}$ in Eq. (3.22b), we obtain

$$
\nabla \times \bar{H}=\mathrm{j} \omega \epsilon_{o}\left(\epsilon_{r}^{\prime}-\mathrm{j} \epsilon_{r}^{\prime \prime}\right) \bar{E}
$$

or

$$
\nabla \times \bar{H}=\bar{J}_{c e}+\bar{J}_{d e}
$$

where $\bar{J}_{c e}$ is the effective electric conduction current density, and $\bar{J}_{d e}$ is the effective displacement electric current density.

From these equations, the conservation of complex power equation for a source-free region, for the unperturbed problem, may be derived as

$$
\int_{S}\left(\frac{1}{2} \bar{E} \times \overline{\boldsymbol{B}}^{*}\right) \cdot d \overline{\mathbf{s}}=-\mathrm{j} 2 \omega \int_{V}\left(\frac{1}{4} \mu_{o}|\overline{\boldsymbol{H}}|^{2}-\frac{1}{4} \hat{\epsilon}_{a}^{*}|\overline{\mathbf{E}}|^{2}\right) d v
$$

where $S$ is the aperture surface area, $V$ is the volume enclosed by the cavity structure, and $\hat{\epsilon}_{a}$ is the apparent value of the dielectric complex permittivity. This equation says that the exciting complex power (in watts) is stored as electric and magnetic energy in the dielectric, and dissipated in the dielectric. The power dissipated in this unperturbed problem will be represented by $P_{d}$. There will be two parts to the power dissipated in the cavity. One part is the power dissipated 
in the lossy dielectric $\left(P_{d d}\right)$ and another in the conductor cavity walls $\left(P_{d c}\right)$ such that

$$
P_{d}=P_{d d}+P_{d c}
$$

For the perturbed problem, the cavity is enclosed with imperfectly conducting walls having finite conductivity $\sigma$, that results in a surface impedance as shown in the $\mathrm{TE}_{10}$ example, given by

$$
Z_{s}=R_{s}+\mathrm{j} X_{s} \simeq \sqrt{\frac{\mathrm{j} \omega \mu}{\sigma}}=\sqrt{\frac{\omega \mu}{2 \sigma}}(1+\mathrm{j})
$$

The conservation of complex power equation for the perturbed problem may be written as

$$
\begin{array}{r}
\int_{S}\left(\frac{1}{2} \bar{E}_{p} \times \bar{H}_{p}^{*}\right) \cdot d \bar{s}=-\mathrm{j} 2 \omega \int_{V}\left(\frac{1}{4} \mu_{o}\left|\bar{H}_{p}\right|^{2}-\frac{1}{4} \hat{\epsilon}_{e}^{*}\left|\bar{E}_{p}\right|^{2}\right) d v \\
-\frac{Z_{s}}{2} \int_{A_{w}}\left|\bar{H}_{p \text { tang }}\right|^{2} d s
\end{array}
$$

where $A_{w}$ is the surface of the conducting walls, and $\hat{\epsilon}_{e}$ is the estimated value of the dielectric complex permittivity. This equation shows that the exciting power is stored as energy and dissipated in both the dielectric and the conducting walls.

For high conductivity metals, with the skin depth of the conductor much less than the conductor thickness, the fields inside the cavity are only slightly perturbed from the unperturbed problem case. An additional assumption that has to be checked is that the skin depth of the conductor has to be much smaller than 
the significant radii of curvature (cavity radius $c$ ). If these assumptions hold, $\bar{E}_{p}$ and $\overline{\boldsymbol{H}}_{p}$ may be approximated by $\overline{\boldsymbol{E}}$ and $\overline{\boldsymbol{H}}$. On the cavity walls the electric field is no longer zero, but we assume that the magnetic field $\bar{H}_{p \text { tang }}$ has only changed slightly, so it may be replaced by $\overline{\boldsymbol{H}}_{\text {tang }}$ of the unperturbed problem. After these substitutions, Eq. (3.26) may be rewritten as

$$
\begin{aligned}
\int_{S}\left(\frac{1}{2} \bar{E} \times \overline{\boldsymbol{H}}^{*}\right) \cdot d \bar{s}=-\mathrm{j} 2 \omega \int_{V}\left(\frac{1}{4} \mu_{o}|\overline{\boldsymbol{H}}|^{2}\right. & \left.-\frac{1}{4} \hat{\epsilon}_{e}^{*}|\overline{\boldsymbol{E}}|^{2}\right) d v \\
& -\frac{Z_{s}}{2} \int_{A_{w}}\left|\overline{\boldsymbol{H}}_{\text {tang }}\right|^{2} d s .
\end{aligned}
$$

Subtracting Eq. (3.24) from Eq. (3.27) we obtain

$$
\hat{\epsilon}_{a}^{*}-\hat{\epsilon}_{e}^{*}=\frac{-Z_{s} \int_{S}\left|\overline{\boldsymbol{H}}_{\text {tang }}\right|^{2} d s}{\mathrm{j} \omega \int_{V}|\overline{\mathbf{E}}|^{2} d v} .
$$

This perturbation equation has quadratic nature, which is typical for the perturbation approach.

Separating the real and imaginary parts of Eq. (3.28), the change in the real and imaginary parts of the complex permittivity, with equal real and imaginary parts of the conductor surface impedance, may be found as

$$
\epsilon_{r a}^{\prime \prime}-\epsilon_{r e}^{\prime \prime}=-\left(\epsilon_{r a}^{\prime}-\epsilon_{r e}^{\prime}\right)=\frac{R_{s} \int_{S}\left|\overline{\boldsymbol{H}}_{\text {tang }}\right|^{2} d s}{\epsilon_{0} \omega \int_{V}|\overline{\bar{E}}|^{2} d v}
$$


According to this perturbation equation, the estimated value of the dielectric loss will be lower than the apparent value, while the estimated value of the dielectric constant will be higher than the apparent value. The low-loss dielectric in the cavity has a dielectric constant $\left(\epsilon_{r}^{\prime}\right)$ much higher than the related dielectric loss term $\left(\epsilon_{r}^{\prime \prime}\right)$. Thus, the change in the dielectric constant is expected to be negligible relative to the change in the dielectric loss. This small change in the dielectric constant thus becomes one check on the perturbation assumption.

The change in the dielectric loss will be the only one carried out, and may be rewritten as

$$
\frac{\epsilon_{r a}^{\prime \prime}-\epsilon_{r e}^{\prime \prime}}{\epsilon_{r a}^{\prime \prime}}=\frac{R_{s} \int_{S}\left|\overline{\boldsymbol{H}}_{t a n g}\right|^{2} d s}{\int_{V} \omega \epsilon_{0} \epsilon_{r a}^{\prime \prime}|\bar{E}|^{2} d v}=\frac{R_{s} \int_{S}\left|\overline{\boldsymbol{H}}_{t a n g}\right|^{2} d s}{\operatorname{Re}\left\{\int_{S}\left(\bar{E} \times \overline{\boldsymbol{H}}^{*}\right) \bullet d s\right\}}
$$

or

$$
\frac{\epsilon_{r a}^{\prime \prime}-\epsilon_{r e}^{\prime \prime}}{\epsilon_{r a}^{\prime \prime}}=\frac{\text { Real power loss in the cavity conductor walls }}{\text { Real power absorbed by the cavity }}=\frac{P_{d c}}{P_{d}},
$$

where $P_{d}$ is the real power absorbed by the cavity, and $P_{d c}$ is the real power loss in the conductor walls.

This discussion leads to the conclusion that the difference between the estimated value of the dielectric loss term and the apparent value from the unperturbed problem is equal to the ratio of the real power dissipated in the imperfectly conducting walls of the perturbed problem to the total real exciting power in the cavity for the unperturbed problem. An equivalent form enables us to 
estimate the perturbed dielectric loss $\epsilon_{r e}^{\prime \prime}$ as

$$
\epsilon_{r e}^{\prime \prime}=\frac{P_{d}-P_{d c}}{P_{d}} \epsilon_{r a}^{\prime \prime}
$$

To get the estimated dielectric loss tangent from the apparent loss tangent, we simply divide both sides of Eq. (3.31) with the dielectric constant $\epsilon_{r}^{\prime}$.

\subsubsection{Real power absorbed by the unperturbed cavity}

In evaluating the power absorbed by the unperturbed cavity, the measured reflection coefficient of the cavity structure will be used. The ratio of the $z$ directed time-averaged transmitted power density $\left(S_{z}^{t}\right)$ to the $z$-directed timeaveraged incident power density $\left(S_{z}^{i}\right)$ is proportional to the magnitude of the transmission coefficient squared and weighted by the intrinsic impedances of the two media, which in terms of the magnitude of the reflection coefficient is given as

$$
S_{z}^{t}=\left(1-\left|s_{11}\right|^{2}\right) S_{z}^{i}
$$

For unity incident power, the incident magnetic field at the aperture is given by Eq. (2.46) as

$$
H_{\phi}^{i}=\frac{1}{\rho \sqrt{\eta_{o} \pi \ln (b / a)}}
$$

and the incident electric field at the aperture is

$$
E_{\rho}^{i}=\frac{\sqrt{\eta_{\mathrm{o}}}}{\rho \sqrt{\pi \ln (b / a)}}
$$


The incident power is determined by the integration of the incident $z$-directed power density over the aperture surface given as

$$
P^{i}=\int_{S}\left(\frac{1}{2} \bar{E} \times \bar{H}^{*}\right) \cdot d \bar{s}=\int_{0}^{2 \pi} \int_{a}^{b} \frac{1}{2 \pi \ln (b / a)} \frac{1}{\rho^{2}} \rho d \rho d \phi
$$

which will give unity incident power. So the transmitted power to the cavity or the dissipated power (in Watts) in the cavity is

$$
P_{d}=\left(1-\left|s_{11}\right|^{2}\right)
$$

\subsubsection{Real power dissipated in the walls of the perturbed cavity}

The power dissipated in the cavity walls will be evaluated using Eq. (3.17) discussed in section (3.2) and given as

$$
P_{d}=\frac{R_{s}}{2} \int_{A_{w}}\left|\bar{H}_{t a n g}\right|^{2} d s
$$

with $A_{w}$ representing the cavity walls surface. The total power dissipated in the walls is the sum of the powers dissipated, as shown on Fig. 3.3, in the front $\left(P_{d f}\right)$, back $\left(P_{d b}\right)$, and side $\left(P_{d s}\right)$ walls as given by

$$
P_{d c}=P_{d f}+P_{d b}+P_{d s}
$$

For the front wall, the surface has two parts, as shown in Fig 3.3, one is a circle covering the center conductor with radius $a$, and the other is a ring with inner radius $b$ and outer radius $c$. The only component of the magnetic field 


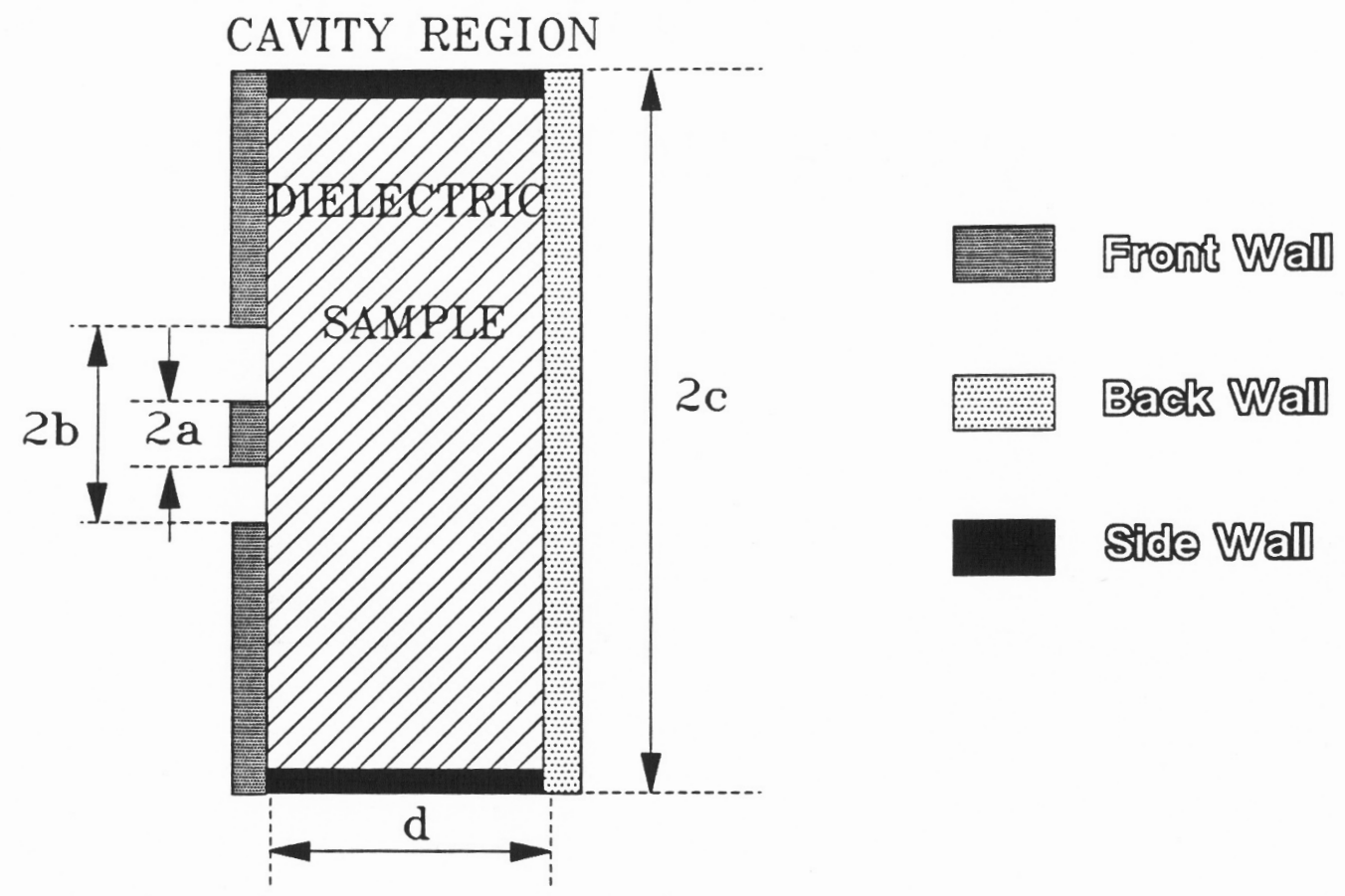

Figure 3.3. Walls of the cavity structure. 
tangential to this surface is the $\phi$-component of the magnetic field in the cavity region, so the power dissipated in the front wall will be evaluated according to Eq. (3.17) is

$$
\begin{aligned}
P_{d f}=\frac{R_{s}}{2}\left\{\int_{0}^{2 \pi} \int_{0}^{a}\left(H_{\phi}^{B} H_{\phi}^{B *}\right)_{z=0} \rho \mathrm{d} \rho \mathrm{d} \phi\right. \\
\left.\quad+\int_{0}^{2 \pi} \int_{b}^{c}\left(H_{\phi}^{B} H_{\phi}^{B *}\right)_{z=0} \rho d \rho d \phi\right\} .
\end{aligned}
$$

The $\phi$-component of the magnetic field in the cavity is given as a sum of all possible modes as given by Eq. (2.62) as

$$
H_{\phi}^{B}=-\mathrm{j} \omega \hat{\epsilon} \sum_{m=1}^{M} \sum_{n=0}^{N-1} \frac{V_{n} C_{m n}}{k_{z_{m}}^{B}} J_{1}\left(k_{\rho_{m}}^{B} \rho\right) \cos \left(k_{z_{m}}^{B}(z-d)\right)
$$

where the coefficients $V_{n}$ are the expansion coefficients of the magnetic current distribution on the aperture $\left(M_{s}\right)$ in terms of the expansion functions $M_{n}$ as

$$
M=\sum_{n} V_{n} M_{n}
$$

and the coefficients $C_{m n}$ are given by Eq. (2.60) as

$$
C_{m n}=\frac{-2}{c^{2} J^{2}{ }_{1}\left(k_{\rho_{m}}^{B} c\right) \sin \left(k_{z_{m}}^{B} d\right)} \int_{a}^{b} M_{n} J_{1}\left(k_{\rho_{m}}^{B} \rho\right) \rho d \rho .
$$

The constants $k_{\rho_{m}}^{B}$ must satisfy the condition given by Eq. (2.43) as

$$
J_{0}\left(k_{\rho_{m}}^{B} c\right)=0
$$


and $k_{z_{m}}^{B}$ are computed from Eq. (2.44) as

$$
k_{z_{m}}^{B}=\sqrt{\omega^{2} \hat{\epsilon} \mu_{0}-\left(k_{\rho_{m}}^{B}\right)^{2}} .
$$

By introducing new coefficients $C_{m n}^{\prime}$ Eqs. (3.39) and (3.40) may be rewritten as

$$
H_{\phi}^{B}=\mathrm{j} \omega \hat{\epsilon} \sum_{m=1}^{M} \sum_{n=0}^{N-1} \frac{V_{n} C_{m n}^{\prime} J_{1}\left(k_{\rho_{m}}^{B} \rho\right) \cos \left(k_{z_{m}}^{B}(z-d)\right)}{\sqrt{\pi} c J_{1}\left(k_{\rho_{m}}^{B} c\right) \sin \left(k_{z_{m}}^{B} d\right) k_{z_{m}}^{B}}
$$

where

$$
C_{m \bar{n}}^{\prime}-\left[\sqrt{\pi} c J_{1}\left(k_{\rho_{m}}^{B} c\right) \sin \left(k_{z_{m}}^{B} d\right)\right] C_{m n}
$$

Each mode in the cavity propagates energy as if it exists alone. Hence the total power dissipated is a summation of the powers dissipated by each mode.

Because of the orthogonality of the mode vectors, there will be no cross terms when we integrate over the entire cavity cross section. Since the magnetic field in the transmission line and the cavity regions are equivalent over the aperture, Eq. (3.38) may be rewritten, to use this orthogonality, as

$$
\begin{aligned}
P_{d f}=\frac{R_{s}}{2}\left\{\int_{0}^{2 \pi} \int_{0}^{c}\left(H_{\phi}^{B} H_{\phi}^{B *}\right)_{z}=0\right. & \rho \mathrm{d} \rho \mathrm{d} \phi \\
& \left.-\int_{0}^{2 \pi} \int_{a}^{b}\left(H_{\phi}^{A} H_{\phi}^{A *}\right)_{z=0} \rho d \rho d \phi\right\},
\end{aligned}
$$

where the superscript $A$ stands for the transmission line region, and $B$ stands for the cavity region. Substituting the $\phi$-component of the magnetic field for both 
regions in Eq. (3.43), we obtain

$$
\begin{gathered}
\left.P_{d f}=R_{s} \sum_{m=1}^{M}\left\{\left|\frac{j \omega \hat{\epsilon} \cos \left(k_{z_{m}}^{B} d\right)}{k_{z_{m}}^{B} \sin \left(k_{z_{m}}^{B} d\right)}\right|^{2}\right\} \sum_{n=0}^{N-1}\left|V_{n} C_{m n}^{\prime}\right|^{2}\right\}\left\{\int_{0}^{c} \frac{J_{1}{ }^{2}\left(k_{\rho_{m}}^{B} \rho\right)}{c^{2} J_{1}^{2}\left(k_{\rho_{m}}^{B}\right)} \rho \mathrm{d} \rho\right\} \\
-\frac{R_{s}}{2} \sum_{n=0}^{N-1}\left|\frac{V_{n}}{\eta_{n}}\right|^{2} \int_{0}^{2 \pi} \int_{a}^{b}\left|h_{n}\right|^{2} \rho d \rho d \phi
\end{gathered}
$$

The functions $h_{n}$ are defined in Eq. (2.53) as

$$
h_{n}(\rho)=\frac{\mathrm{B}_{1}\left(k_{\rho_{n}}^{A} \rho\right)}{\sqrt{\pi\left[b^{2} \mathrm{~B}_{1}^{2}\left(k_{\rho_{n}}^{A} b\right)-a^{2} \mathrm{~B}_{1}^{2}\left(k_{\rho_{n}}^{A} a\right)\right]}}
$$

where $a$ and $b$ are the transmission line radii, and $\mathrm{B}_{1}\left(k_{\rho_{n}}^{A} \rho\right)$ are the Bessel functions given by Eq. (2.28) as

$$
\mathrm{B}_{1}\left(k_{\rho}^{A} \rho\right)=N_{d}\left(k_{\rho}^{A} a\right) J_{1}\left(k_{\rho}^{A} \rho\right)-J_{d}\left(k_{\rho}^{A} a\right) N_{1}\left(k_{\rho}^{A} \rho\right)
$$

The coefficients $k_{\rho_{n}}^{A}$ are obtained from the following equation

$$
\left.N_{0}\left(k_{\rho_{n}}^{A} a\right) J_{0}\left(k_{\rho_{n}}^{A} b\right)=J_{0}\left(k_{\rho_{n}}^{A} a\right) N_{0}\left(k_{\rho_{n}}^{A} b\right)\right]
$$

By evaluating the integrations in Eq. (3.44), we obtain the final closed form given as

$$
P_{d f}=\frac{R_{s}}{2}\left\{\sum_{m=1}^{M}\left\{\left|\frac{j \omega \hat{\epsilon} \cos \left(k_{z_{m}}^{B} d\right)}{k_{z_{m}} \sin \left(k_{z_{m}}^{B} d\right)}\right|^{2}\right\}\left\{\sum_{n=0}^{N-1}\left|V_{n} C_{m n}^{\prime}\right|^{2}\right\}-\sum_{n=0}^{N-1}\left|\frac{V_{n}}{\eta_{n}}\right|^{2}\right\}
$$


The power dissipated in the back wall will be evaluated as the power dissipated in a wall made of a complete circle with radius $c$. The only tangential component of the magnetic field to this wall is the $\phi$-component. So Eq. (3.17) becomes

$$
P_{d b}=\frac{R_{s}}{2} \int_{0}^{2 \pi} \int_{0}^{c}\left(H_{\phi}^{B} H_{\phi}^{B *}\right)_{z=d} \rho d \rho d \phi
$$

Because of the orthogonality of the modes of the magnetic field in the cavity region, there are no cross terms in the evaluation of the power. Substituting the $\phi$ component of the magnetic field of Eq. (3.41) in Eq. (3.46), and evaluating the integration, we obtain

$$
P_{d b}=\frac{R_{s}}{2} \sum_{m=1}^{M}\left\{\left|\frac{j \omega \hat{\epsilon}}{k_{z_{m}}^{B} \sin \left(k_{z_{m}}^{B} d\right)}\right|^{2}\right\}\left\{\sum_{n=0}^{N-1}\left|V_{n} C_{m n}^{\prime}\right|^{2}\right\}
$$

Finally the power dissipated in the side wall is the power dissipated in a ring with a thickness $(d)$ being that of the dielectric sample in the cavity structure. As a first estimate, the cross terms will be neglected to get the power dissipated in the side wall.

Again the only tangential component to the side wall is the $\phi$-component of the magnetic field. Using Eq. (3.17), the power dissipated in the side wall is evaluated as

$$
P_{d s}=\frac{R_{s}}{2} \int_{0}^{d} \int_{0}^{2 \pi}\left(H_{\phi}^{B} H_{\phi}^{B *}\right)_{\rho=c} c d \phi d z
$$

Substituting the $\phi$-component of the magnetic field of Eq. (3.41) in Eq. (3.48), and 
evaluating the integration, the following closed form is obtained:

$$
\begin{gathered}
P_{d s}=\frac{R_{s}}{c} \sum_{m=1}^{M}\left\{\frac{d}{2}\left[\frac{\sinh \left(2 \beta_{m} d\right)}{2 \beta_{m} d}+\frac{\sin \left(2 \alpha_{m} d\right)}{2 \alpha_{m} d}\right]\right\}\left\{\left|\frac{j \omega \hat{\epsilon}}{k_{z_{m}}^{B} \sin \left(k_{z_{m}}^{B} d\right)}\right|^{2}\right\} \\
\left\{\sum_{n=0}^{N-1}\left|V_{n} C_{m n}^{\prime}\right|^{2}\right\}
\end{gathered}
$$

where $\alpha_{m}$ and $\beta_{m}$ are the real and imaginary parts of the propagation constants $k_{z_{m}}^{B}$. Away from radial resonance, the magnitude of the magnetic field in the side wall is negligible compared to its respective value in the front and back walls. So the power dissipated in the side wall is negligible compared to the total power loss in the walls, but it will still be evaluated for checking and will be taken into account. Then neglecting the cross terms, in evaluating the power loss in the side wall, has a minimal effect on the total power loss in the conducting walls, and therefore on the estimation of the dielectric loss tangent.

Once the power dissipated in the unperturbed cavity, and the power dissipated in the imperfectly conducting walls of the perturbed cavity are evaluated, the dielectric loss tangent may be separated from the apparent loss tangent using the perturbation equation discussed earlier in this section and given in Eq. (3.31).

A FORTRAN program was written to compute the estimated dielectric loss tangent, given the apparent dielectric constant and loss tangent, the conductor conductivity, and the measured reflection coefficient magnitude as function of frequency. The FORTRAN program is listed in an Appendix. 


\subsection{SUMMARY}

A perturbation approach has been developed to solve for the attenuation introduced in a rectangular waveguide due to the imperfectly conducting walls of the guide. The developed perturbation approach was then applied to the unperturbed cavity problem analyzed in Chapter 2 with perfectly conducting walls. The perturbation technique takes into account the loss in the imperfectly conducting walls of the cavity structure. The total power loss in the unperturbed problem was computed from the measured reflection coefficient. The power loss in the cavity walls was evaluated using a perturbation approach and separated from the total loss. The dielectric loss tangent was determined from the apparent loss tangent computed from the unperturbed analysis and the ratio of the power loss solely in the dielectric to the total power loss in the cavity. 


\section{CHAPTER 4}

\section{SIMULATION AND EXPERIMENTAL RESULTS}

\subsection{INTRODUCTION}

The perturbation approach has been developed in the previous chapter. It has been applied to the unperturbed problem presented in Chapter 2. This technique may be used to characterize dielectric materials in the microwave frequency range. The method has been implemented on a computer to determine the loss in the cavity walls, given specific conductor properties. The total power dissipated in the cavity has been determined from the magnitude of the measured reflection coefficient. The dielectric loss tangent is estimated from this total power by subtracting the estimated loss in the walls. Simulation for a given problem, showing the process, the measurements, and the results, is presented in Section 4.2. Experimental work is used to verify this method as well as to demonstrate the use of this technique, including dielectric loss determination for two Dupont polymers used in the construction of hybrid integrated circuits and multilayer structures. Experimental results for the Dupont polymer Pyralux ${ }^{\circledR}$, and for low-temperature co-fireable multilayer ceramic (LTCC), known as Green Tape ${ }^{\mathrm{TM}}$ are presented in Sections 4.3 , and 4.4 , respectively.

\subsection{PROBLEM SIMULATION}

A typical problem is simulated in order to check the validity of the loss technique. The cavity structure is formed by enclosing the dielectric in a cylindrical cavity bounded by a low-loss conductor. The dielectric constant was 
chosen to be 5.0, characteristic of typical values. The thickness of the dielectric was assumed to be $0.02 \mathrm{~cm}(8$ mils $)$. The front and back walls were composed of a conductor with conductivity of $6 \times 10^{5} \mathrm{~S} / \mathrm{cm}$ and the edges represent silver paint with conductivity of $2 \times 10^{5} \mathrm{~S} / \mathrm{cm}$.

In order for the apparent dielectric loss tangent to include the loss in the cavity walls, it was determined as shown in Fig. 4.1. This loss tangent consists of a constant value for the dielectric loss plus a conductor loss term which varies as the $3 / 2$ power of frequency as seen from Eqs. (3.16), (3.17), and (3.41). The unperturbed analysis was used to compute the reflection coefficient for the different values of apparent dielectric loss over the frequency range from $45 \mathrm{MHz}$ to $5 \mathrm{GHz}$. The resultant reflection coefficient for the problem is shown in Figs. 4.2, and 4.3. The perturbation analysis was then used to compute the power loss in the cavity from the magnitude of the reflection coefficient using Eq. (3.36) and the power in the walls from Eqs. (3.45), (3.47), and (3.49). The total power dissipated in the cavity as well as the power in the cavity walls are shown in Fig. 4.4. Perturbation is used to estimate the dielectric loss tangent from Eq. (3.31). Fig. 4.5 shows the estimated loss tangent after perturbation as well as the apparent loss tangent for the unperturbed problem. The estimated loss tangent demonstrates a constant value as expected. The method does not explicitly impose any frequency dependence model for the dielectric.

The technique separates the conductor loss from the total cavity loss to improve the estimate of the dielectric loss. The next two sections consider the characterization of typical dielectric materials used in the microelectronics industry. 


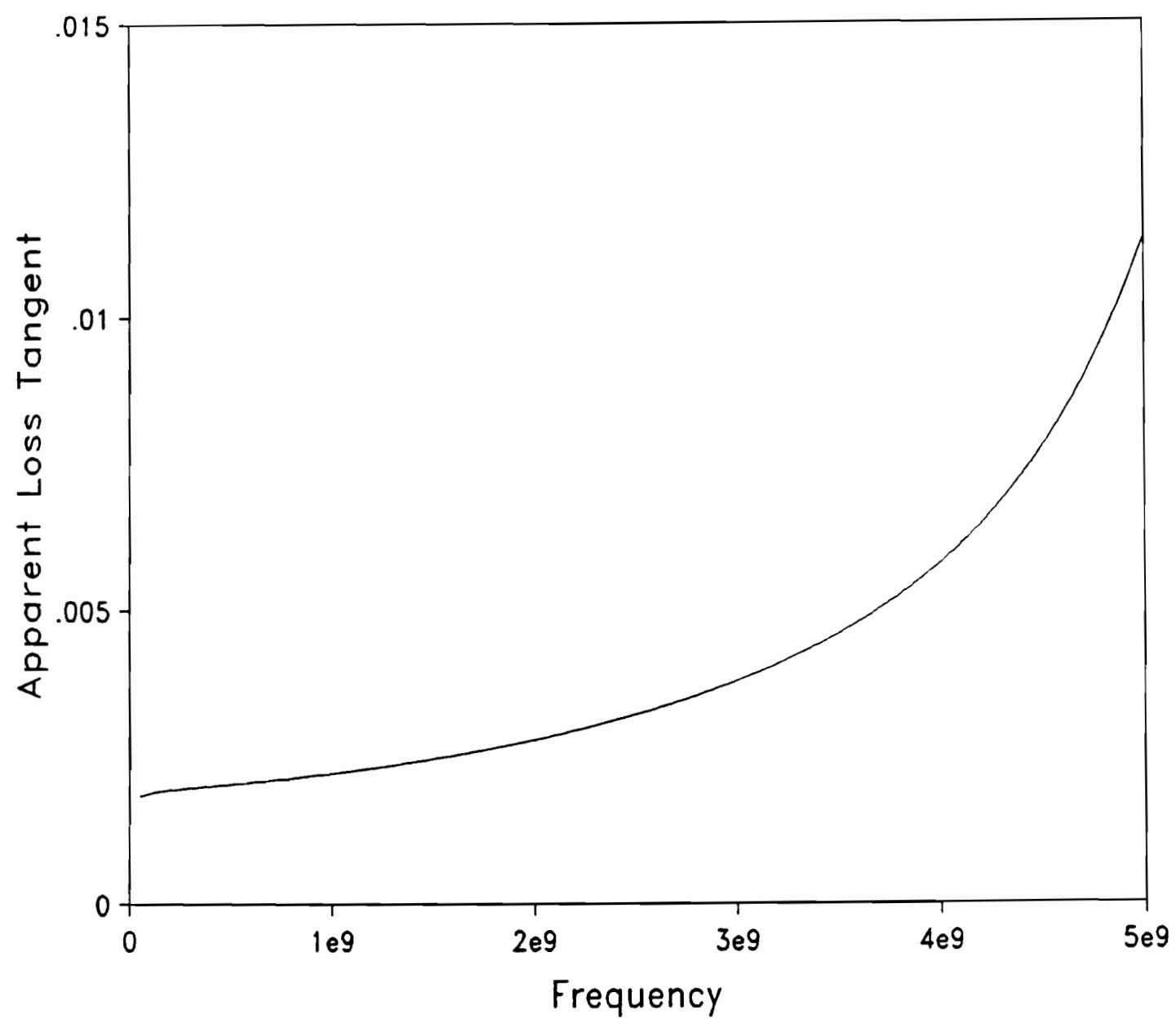

Figure 4.1. Apparent dielectric loss tangent versus frequency in $\mathrm{Hz}$, for the simulated problem with $\epsilon^{\prime}=5.0, d=.02 \mathrm{~cm}$, $\sigma_{\mathrm{F}}=\sigma_{\mathrm{B}}=6 \times 10^{5} \mathrm{~S} / \mathrm{cm}$, and $\sigma_{\mathrm{E}}=2 \times 10^{5} \mathrm{~S} / \mathrm{cm}$. 


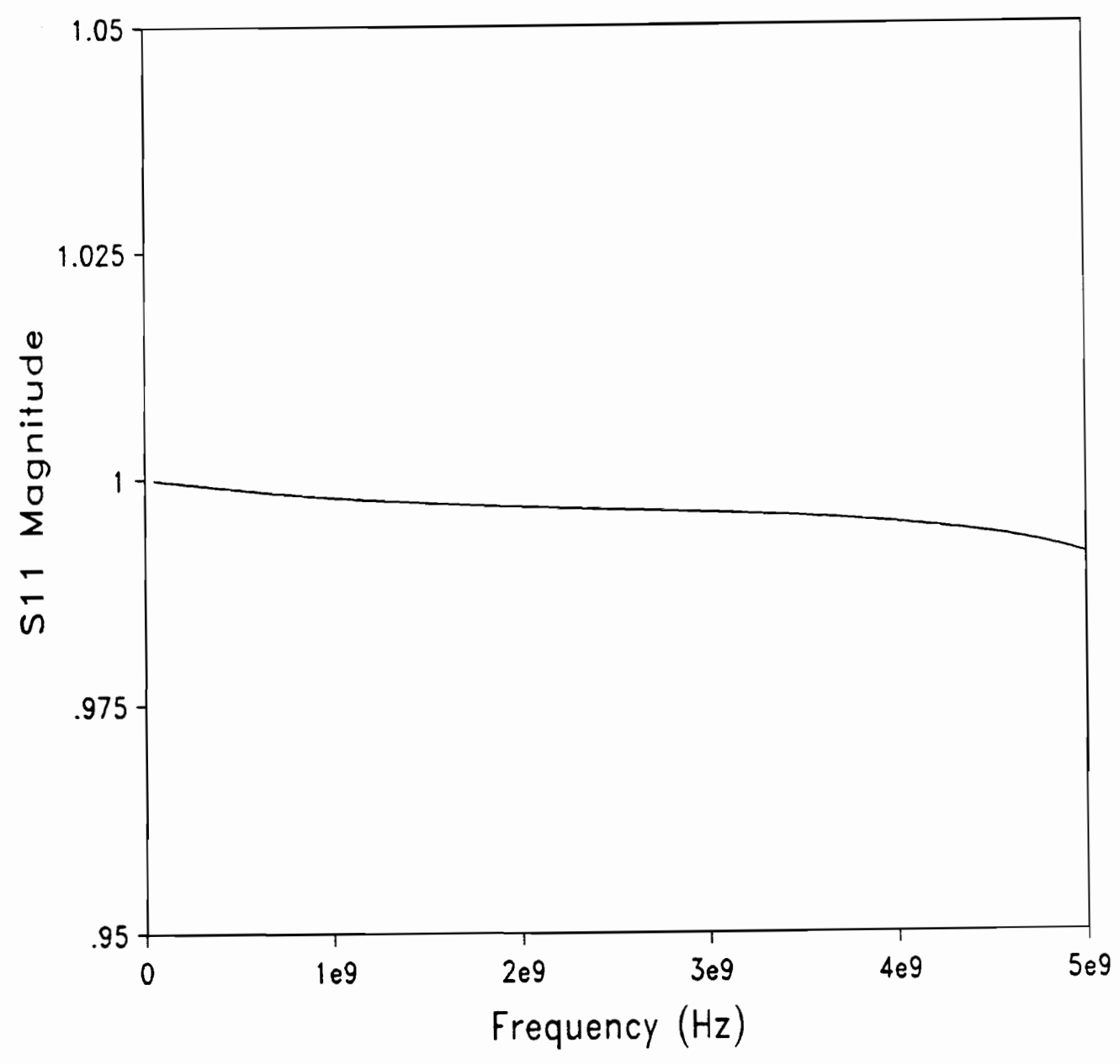

Figure 4.2. Reflection coefficient (magnitude) versus frequency in $\mathrm{Hz}$, for the simulated problem with $\epsilon^{\prime}=5.0, d=.02 \mathrm{~cm}$, $\sigma_{\mathrm{F}}=\sigma_{\mathrm{B}}=6 \times 10^{5} \mathrm{~S} / \mathrm{cm}$, and $\sigma_{\mathrm{E}}=2 \times 10^{5} \mathrm{~S} / \mathrm{cm}$. 


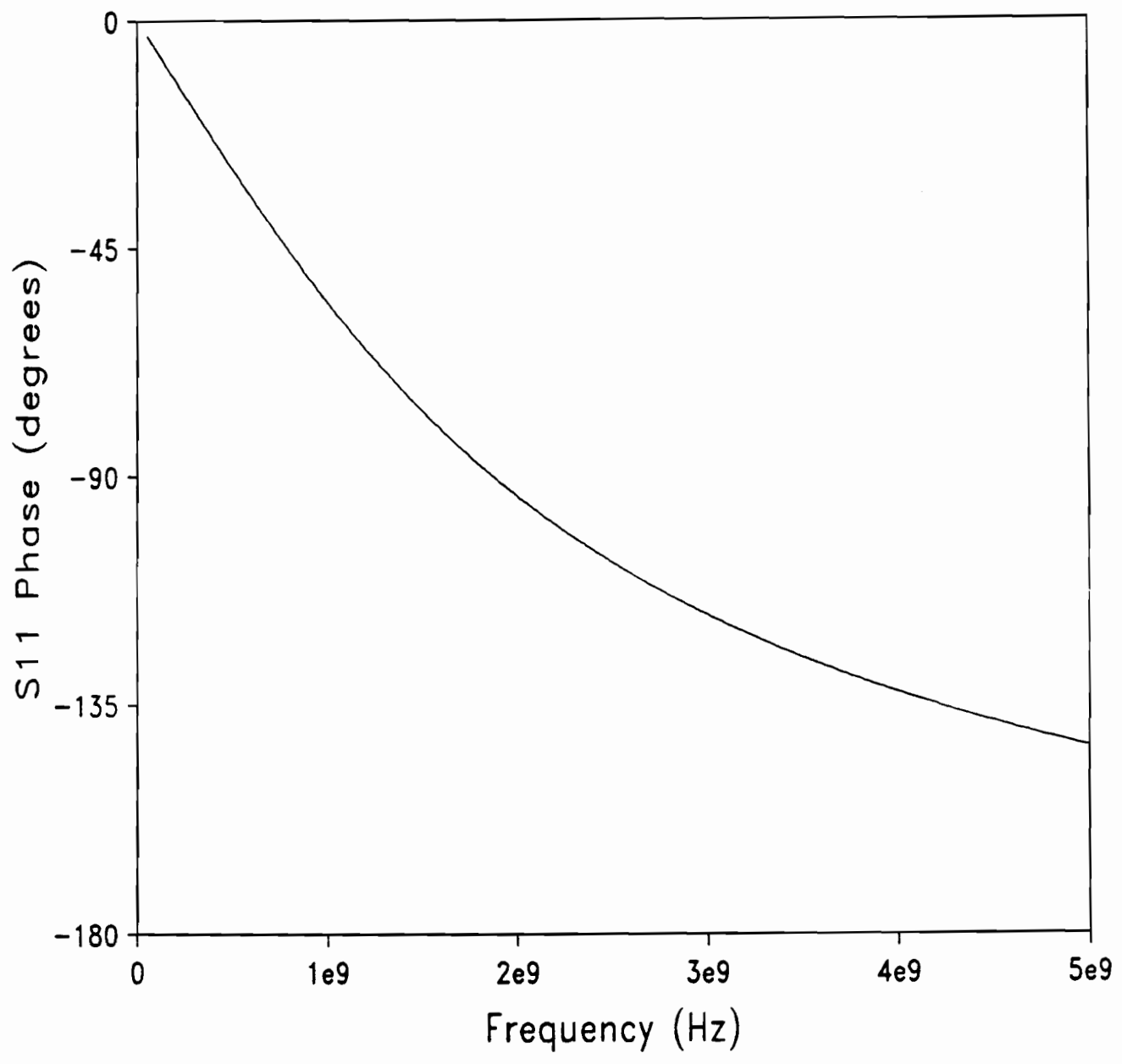

Figure 4.3. Reflection coefficient (phase) versus frequency in $\mathrm{Hz}$, for the simulated problem with $\epsilon^{\prime}=5.0, d=.02 \mathrm{~cm}$, $\sigma_{\mathrm{F}}=\sigma_{\mathrm{B}}=6 \times 10^{5} \mathrm{~S} / \mathrm{cm}$, and $\sigma_{\mathrm{E}}=2 \times 10^{5} \mathrm{~S} / \mathrm{cm}$. 


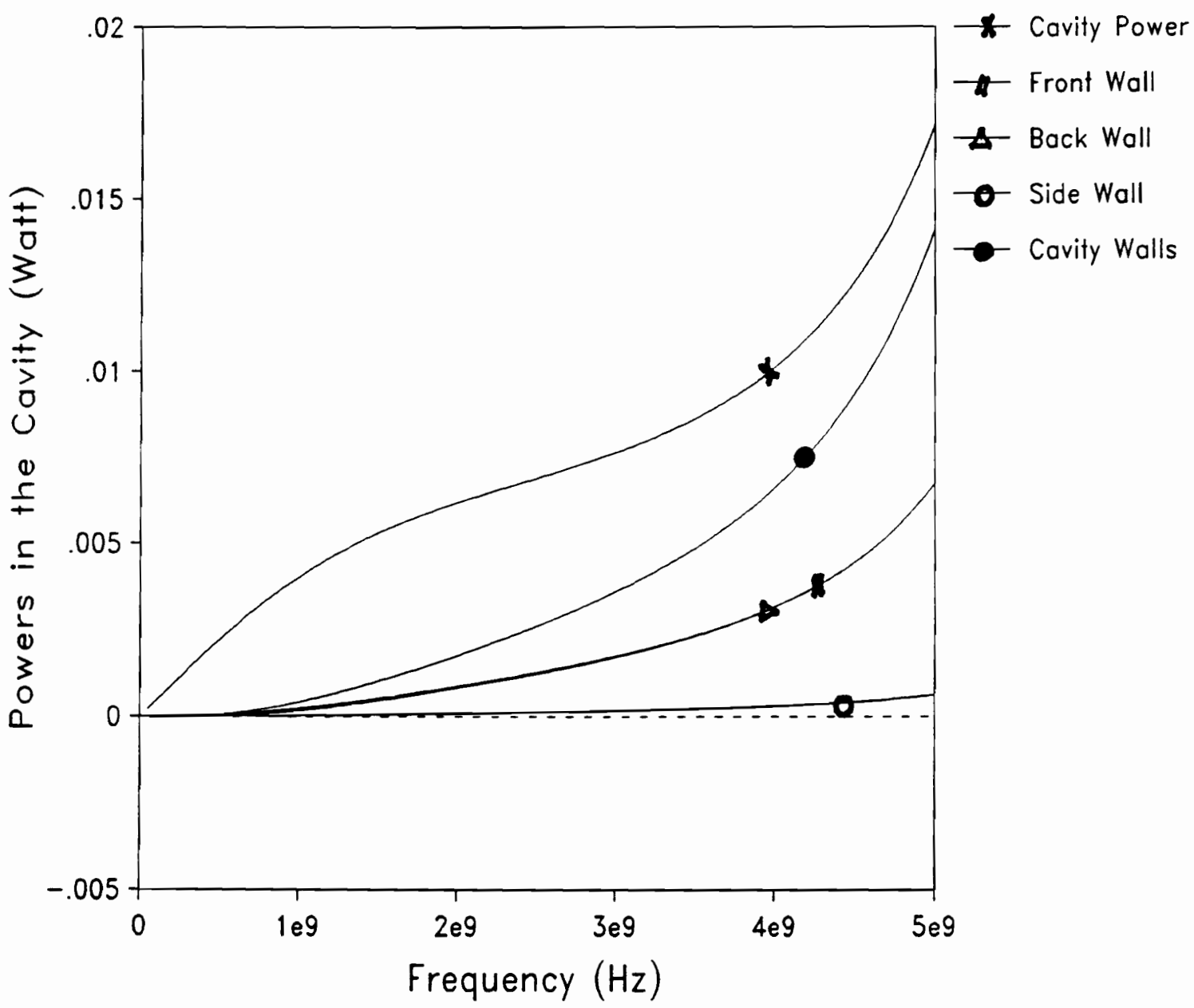

Figure 4.4. The total power loss in the cavity, the power loss in the front, back, and side wall, and the total power loss in the cavity walls versus frequency in $\mathrm{Hz}$, for the simulated problem with $\epsilon^{\prime}=5.0, d=.02 \mathrm{~cm}, \sigma_{\mathrm{F}}=\sigma_{\mathrm{B}}=6 \times 10^{5} \mathrm{~S} / \mathrm{cm}$, and $\sigma_{\mathrm{E}}=2 \times 10^{5} \mathrm{~S} / \mathrm{cm}$. 


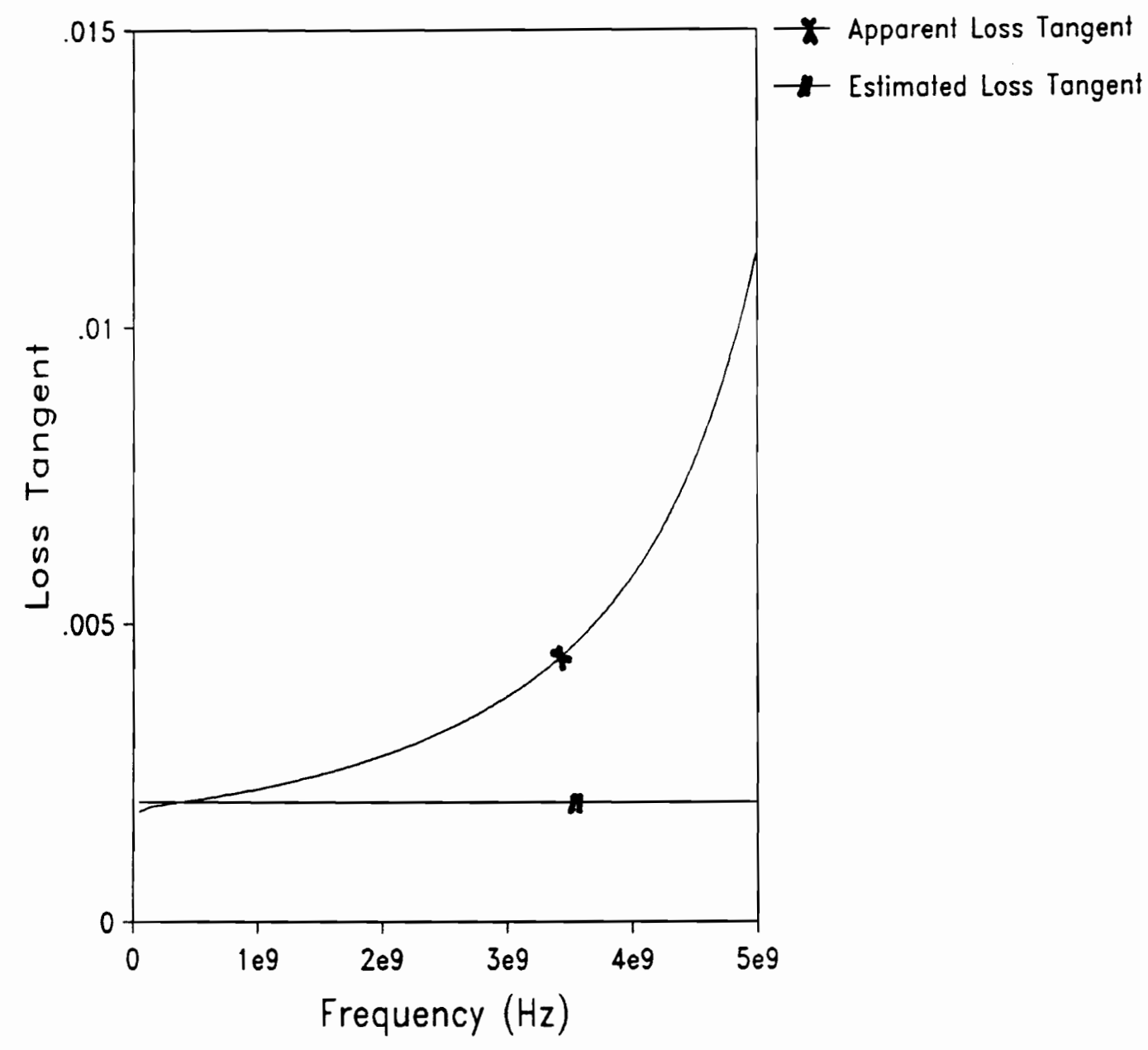

Figure 4.5. The apparent and estimated dielectric loss tangent versus frequency in $\mathrm{Hz}$, for the simulated problem with $\epsilon^{\prime}=5.0$, $d=.02 \mathrm{~cm}, \sigma_{\mathrm{F}}=\sigma_{\mathrm{B}}=6 \times 10^{5} \mathrm{~S} / \mathrm{cm}$, and $\sigma_{\mathrm{E}}=2 \times 10^{5} \mathrm{~S} / \mathrm{cm}$. 


\subsection{DIELECTRIC LOSS DETERMINATION OF PYRALUX ${ }^{\circledR}$}

Pyralux ${ }^{\circledR}$ is a Dupont polymer used in the construction of flexible, hybrid integrated circuits. The perturbation technique is used to separate the conductor loss in order to estimate the dielectric loss tangent. Small corrections to the dielectric constant may also be incorporated, but typically are of little significance.

The Pyralux ${ }^{\circledR}$ samples were fabricated in the microelectronics facilities at Virginia Tech following precise processing steps [S. Riad, A. Riad, and W. Davis, 1990]. The samples were made from a $3^{\prime \prime} \times 3^{\prime \prime}$ layer of Pyralux ${ }^{\circledR}$ LF-9121, with the structure shown in Fig. 4.6. The samples were punched to a radius of $7.458 \mathrm{~cm}$, to fit the stainless steel sleeves of a $7 \mathrm{~mm}$ connector used on the HP $8510 \mathrm{~B}$ network analyzer. The required layout for the top layer of copper forms the electrical contacts to the coaxial cross section of a $7 \mathrm{~mm}$ connector terminating the transmission line, as shown in Fig. 4.7, with an inner conductor radius of $0.15 \mathrm{~cm}$ and an outer conductor radius of $0.35 \mathrm{~cm}$. This layout was designed with a computer aided design (CAD) program. The pattern was plotted on a rubylith with an enlargement ratio of 10 . The pattern was photoreduced and necessary parts were blanked for etching. The copper layer on one side of the samples was then etched using the spray etcher. The other side was left with copper covering the entire back wall of the cavity. The edges of all the samples were painted with silver paint to reduce errors of a possible air gap.

The reflection coefficients $\left(\mathrm{s}_{11}\right)$ of these samples were measured using a frequency-domain fixture on the HP 8510B network analyzer with a one-port full reflection calibration. Figs. 4.8 and 4.9 show typical reflection coefficient

measurements for the Pyralux ${ }^{\circledR}$ samples. The functional relationship between the reflection coefficient and the complex permittivity of the dielectric samples was 

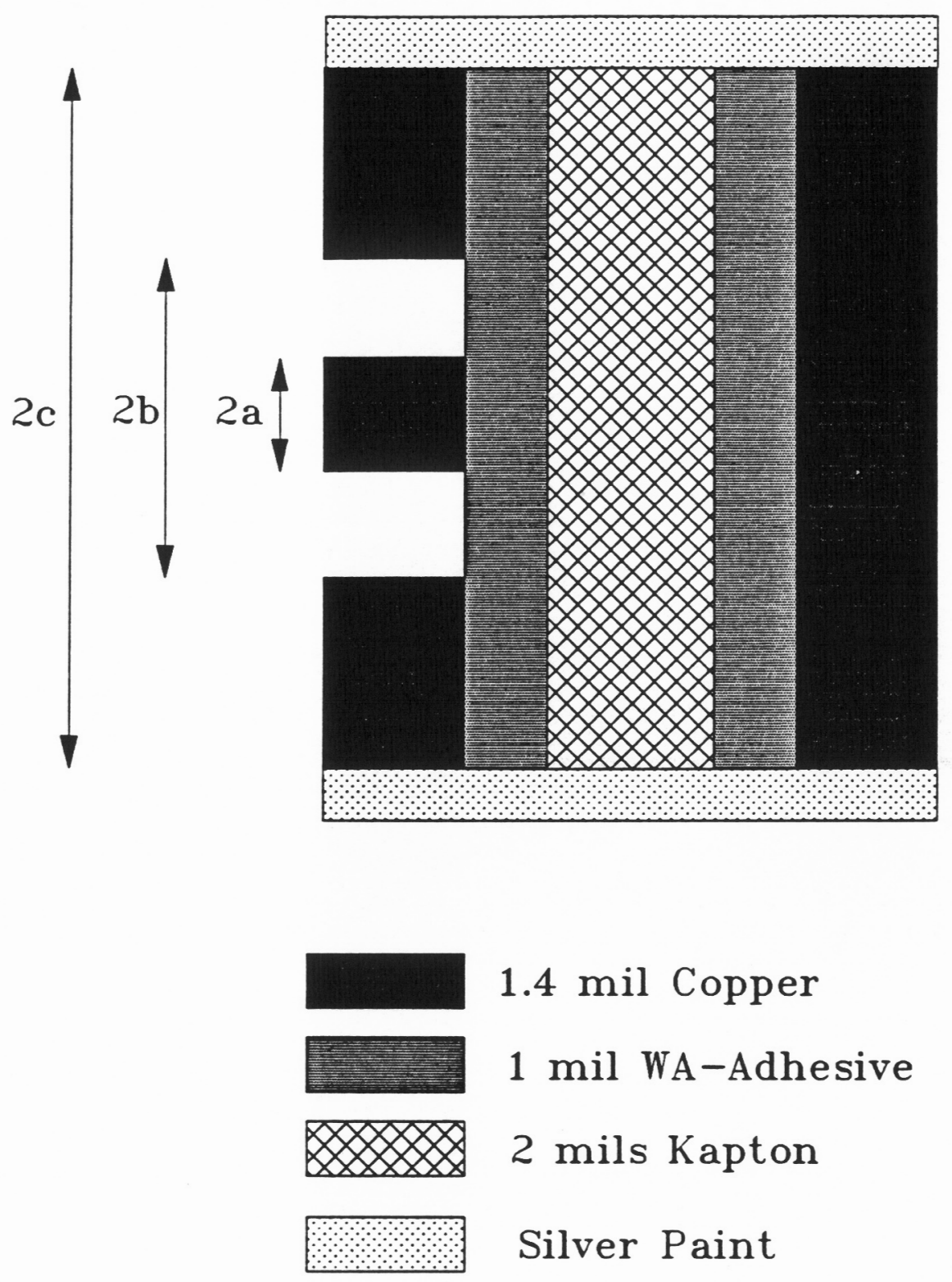

Figure 4.6. $\quad$ Pyralux ${ }^{\circledR}$ samples structure. 


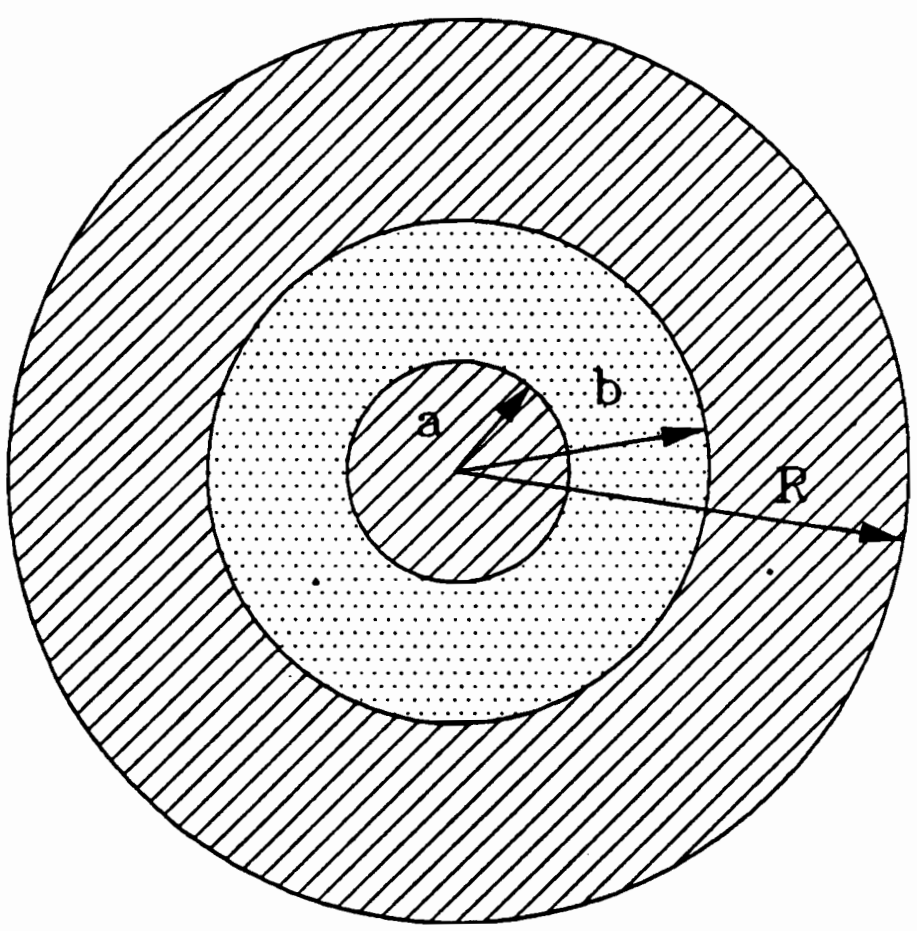

Dielectric

$a=0.15 \mathrm{~cm}$

$\mathrm{b}=0.35 \mathrm{~cm}$

$\square$ Metalization

$\mathrm{R}=0.75 \mathrm{~cm}$

Figure 4.7. $\quad$ Pyralux $^{\circledR}$ samples configuration. 


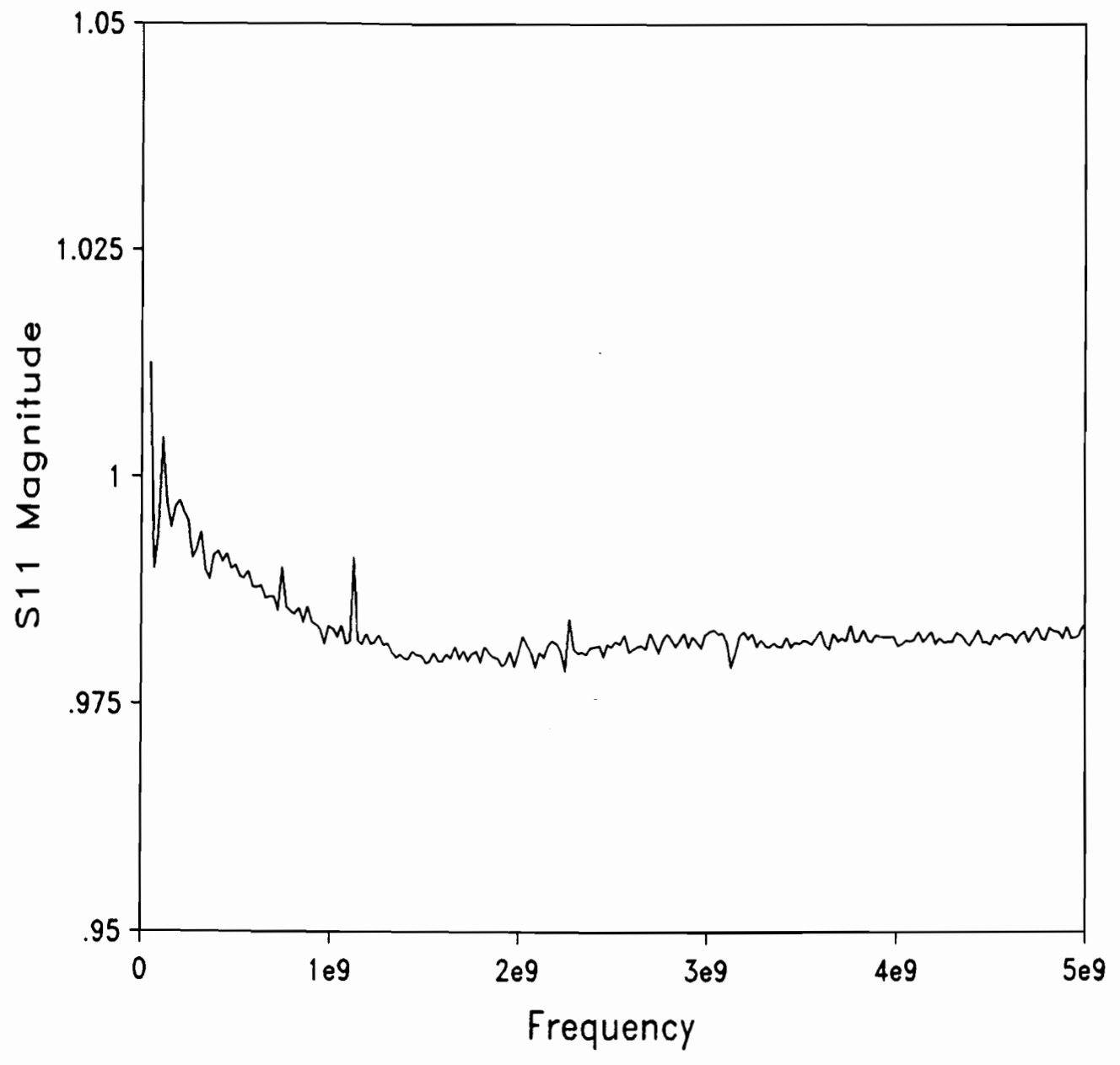

Figure 4.8. Typical Pyralux ${ }^{\circledR}$ reflection coefficient (magnitude). 


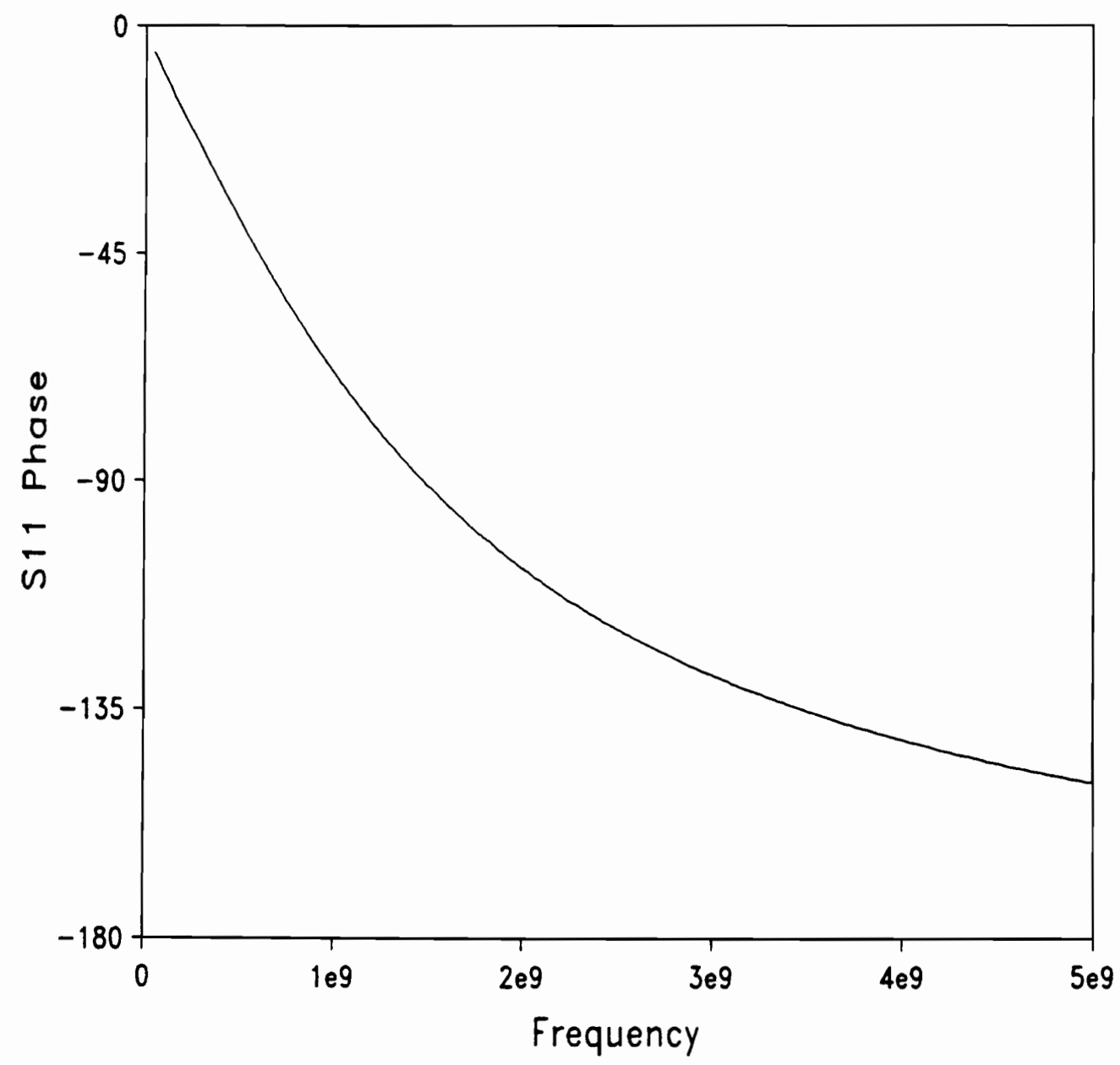

Figure 4.9. Typical Pyralux ${ }^{\circledR}$ reflection coefficient (phase). 
modeled with the full-field analysis of Chapter 2. The samples were analyzed using the unperturbed analysis to determine the dielectric constant and the apparent dielectric loss tangent providing an upper bound on the true dielectric loss tangent. Perturbation is then applied to the results of the unperturbed problem to estimate the true dielectric loss tangent.

The upper bound of the apparent loss tangent is shown in Fig. 4.10 for the frequency range of $45 \mathrm{MHz}$ to $5 \mathrm{GHz}$ starting at 0.015 and increasing to 0.027 . The computed complex unperturbed permittivity was used to compute the power dissipated in the cavity walls as shown in Fig. 4.11. The wall losses are subtracted from the total loss to obtain the power dissipated solely in the dielectric. From the ratio of the power dissipated in the dielectric to the total power dissipated, the dielectric loss tangent of the dielectric is estimated from Eq. (3.31) as shown in Fig. 4.12.

The loss tangent estimated using the perturbation technique demonstrates a reasonable behavior with respect to frequency as the frequency dependent conductor loss has been separated from the apparent loss. The resulting loss tangent agrees with the published results [Riad, 1991] obtained by the Material Characterization Group at $\mathrm{Va}$ Tech using different techniques including transmission line and stripline techniques and by the Dupont Electronics at the Dupont Company using T- and cavity resonators.

\subsection{DIELECTRIC LOSS DETERMINATION OF GREEN TAPETM}

This section presents the results of measurements and application of the perturbation technique to Green Tape ${ }^{\mathrm{TM}}$. The Green Tape ${ }^{\mathrm{TM}}$ dielectric is a Dupont ceramic material used in multilayer technology. 


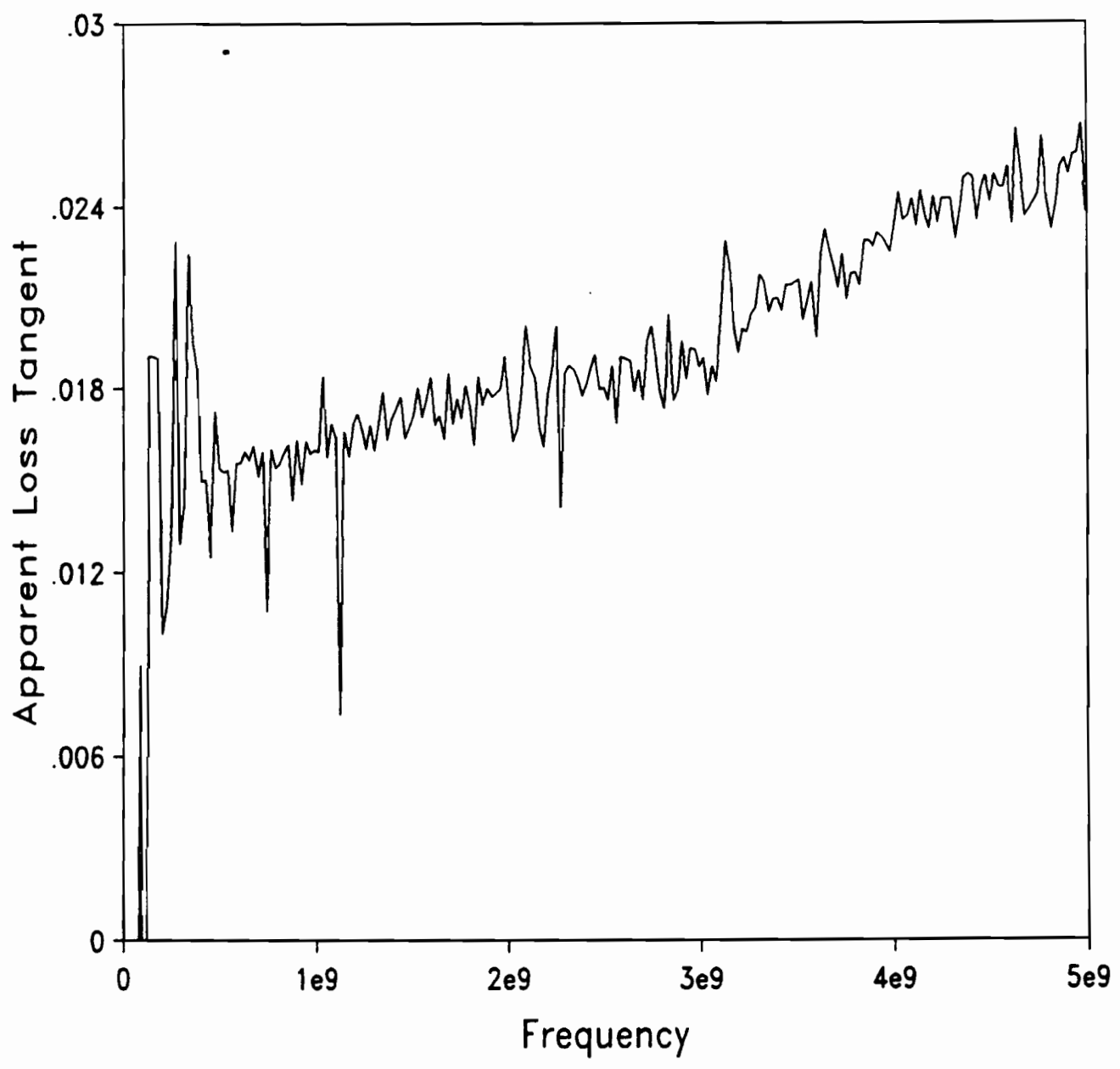

Figure 4.10. Pyralux ${ }^{\circledR}$ apparent loss tangent. 


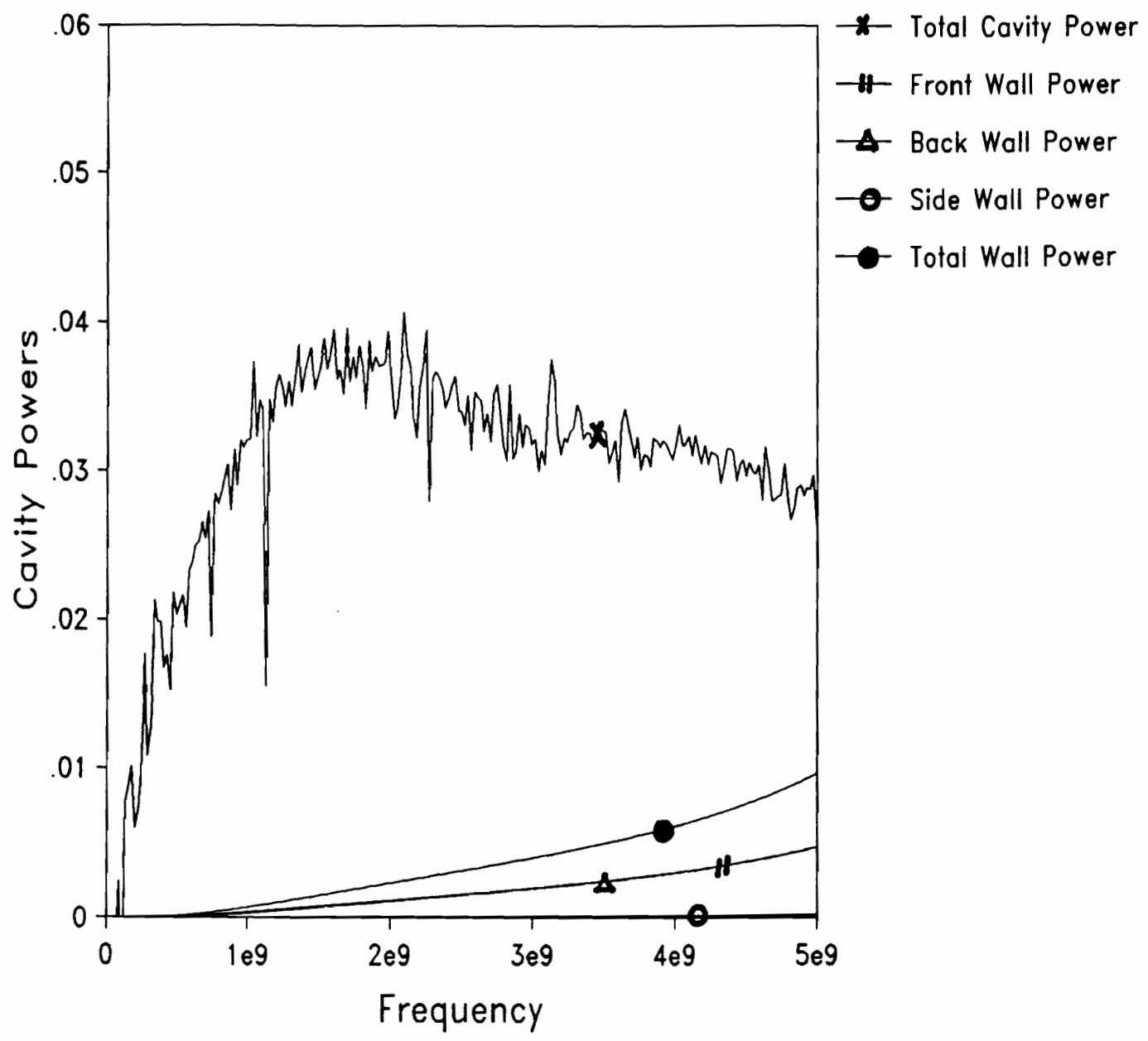

Figure 4.11. Power dissipated in the cavity filled with Pyralux ${ }^{\circledR}$. 


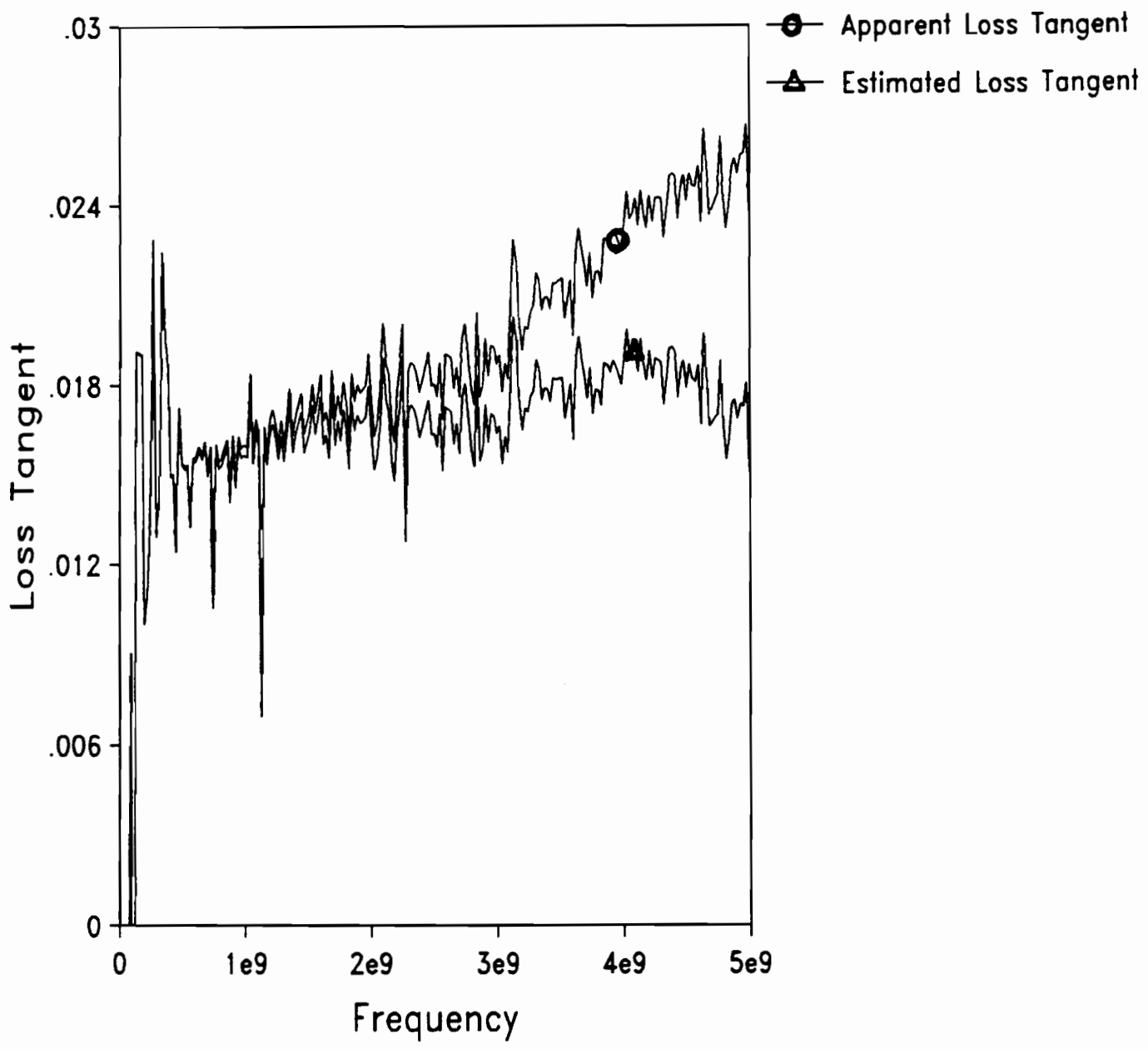

Figure 4.12. Pyralux ${ }^{\circledR}$ estimated and apparent loss tangent . 
The samples were also processed in the microelectronics facility at Va Tech following the material processing and the sample preparation steps listed in the 1990 Dupont Final report [Riad, 1990]. The sample preparation starts by laminating 8 layers of $3^{\prime \prime} \times 3^{\prime \prime}$ squares of Green Tape ${ }^{\mathrm{TM}}$ material, each layer being 3.7 mils thick for a total thickness of 29.6 mils. The dielectric material was punched in a circular shape with a radius of $0.8244 \mathrm{~cm}$ to allow for the shrinkage factor due to the firing process that gives the dielectric its hard ceramic consistence. The final processed samples have a $0.7458 \mathrm{~cm}$ radius to fit the stainless steel sleeves of a $7 \mathrm{~mm}$ connector used on the HP 8510B network analyzer, and a dielectric thickness of 27 mils. The required layout for the conductor layer was designed with a computer aided design (CAD) program with an enlargement ratio of 10 .. The pattern was plotted on a rubylith and photoreduced to create the necessary screens. The dielectric samples were then screen printed, using silver paste on one of the sides of the ceramic samples, to match the cross section of a $7 \mathrm{~mm}$ connector. The other side of the samples and the edges were painted with silver paint to completely enclose the samples and give them the necessary cavity structure for adapting to the end of the transmission line.

The HP 8510B network analyzer was used to measure the reflection coefficient of the samples. This measured reflection coefficient is shown in Figs. 4.13 and 4.14. Using the unperturbed analysis, the complex permittivity of the Green Tape ${ }^{\mathrm{TM}}$ was evaluated for the frequency range of $45 \mathrm{MHz}$ and $4 \mathrm{GHz}$ with a resultant apparent dielectric loss tangent between 0.003 and 0.015 as shown in Fig. 4.15. Using this information in the perturbation analysis, the power in the unperturbed cavity as well as the power in the cavity walls was computed as shown in Fig. 4.16. The resultant estimate of the dielectric loss tangent is shown in Fig. 4.17. 


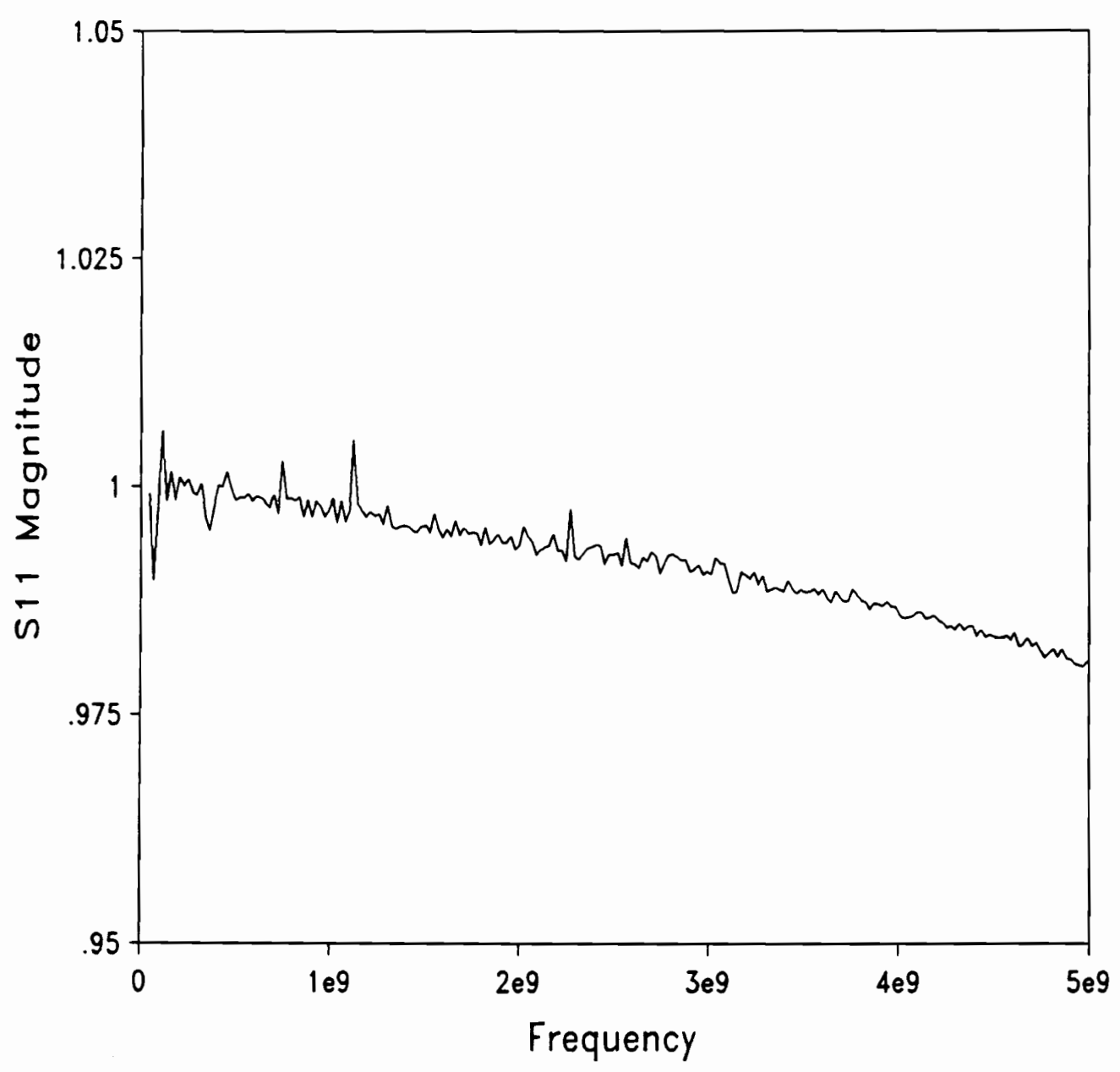

Figure 4.13. Typical Green Tape ${ }^{\mathrm{TM}}$ reflection coefficient (magnitude). 


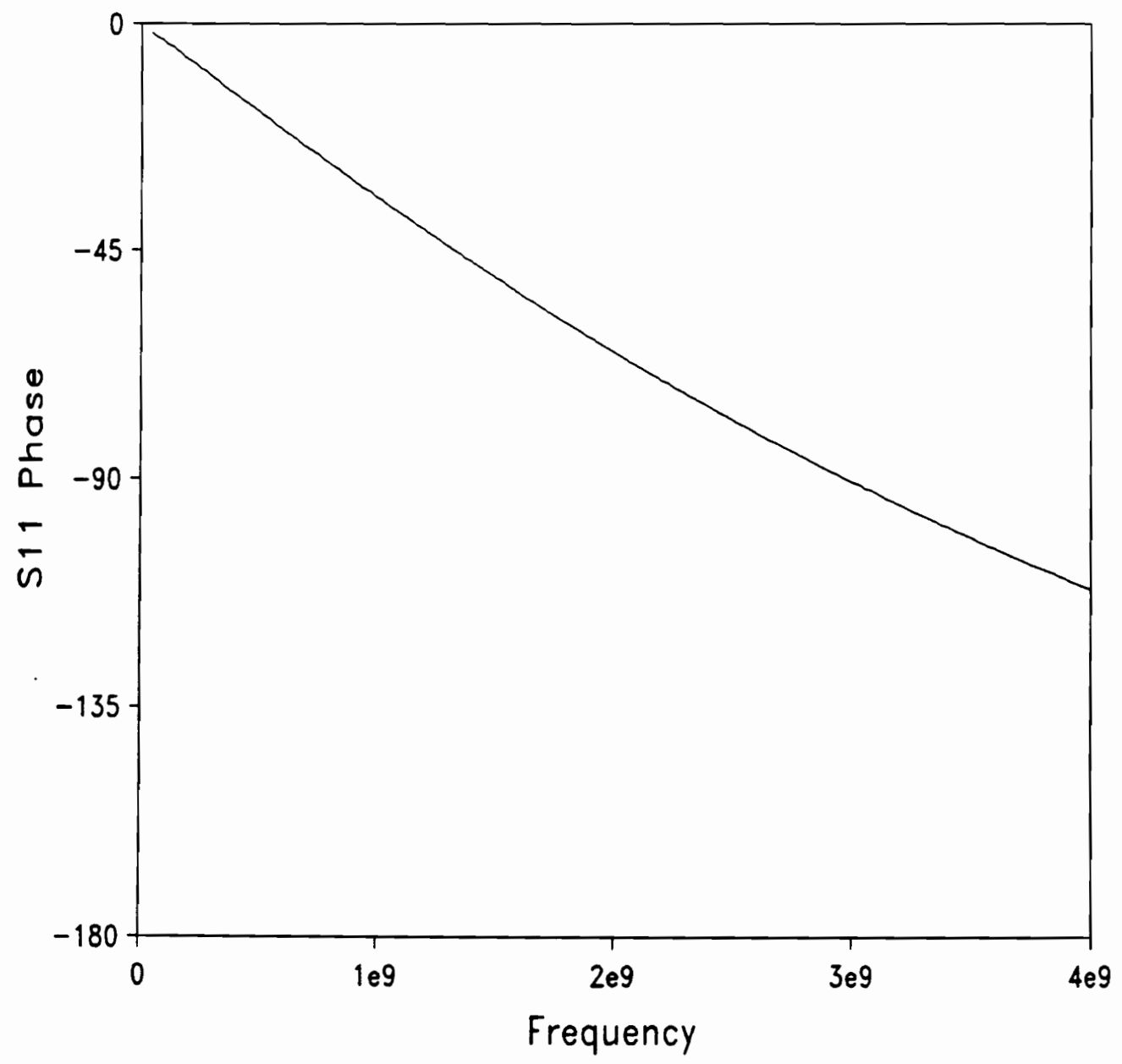

Figure 4.14. Typical Green Tape ${ }^{\mathrm{TM}}$ reflection coefficient (phase). 


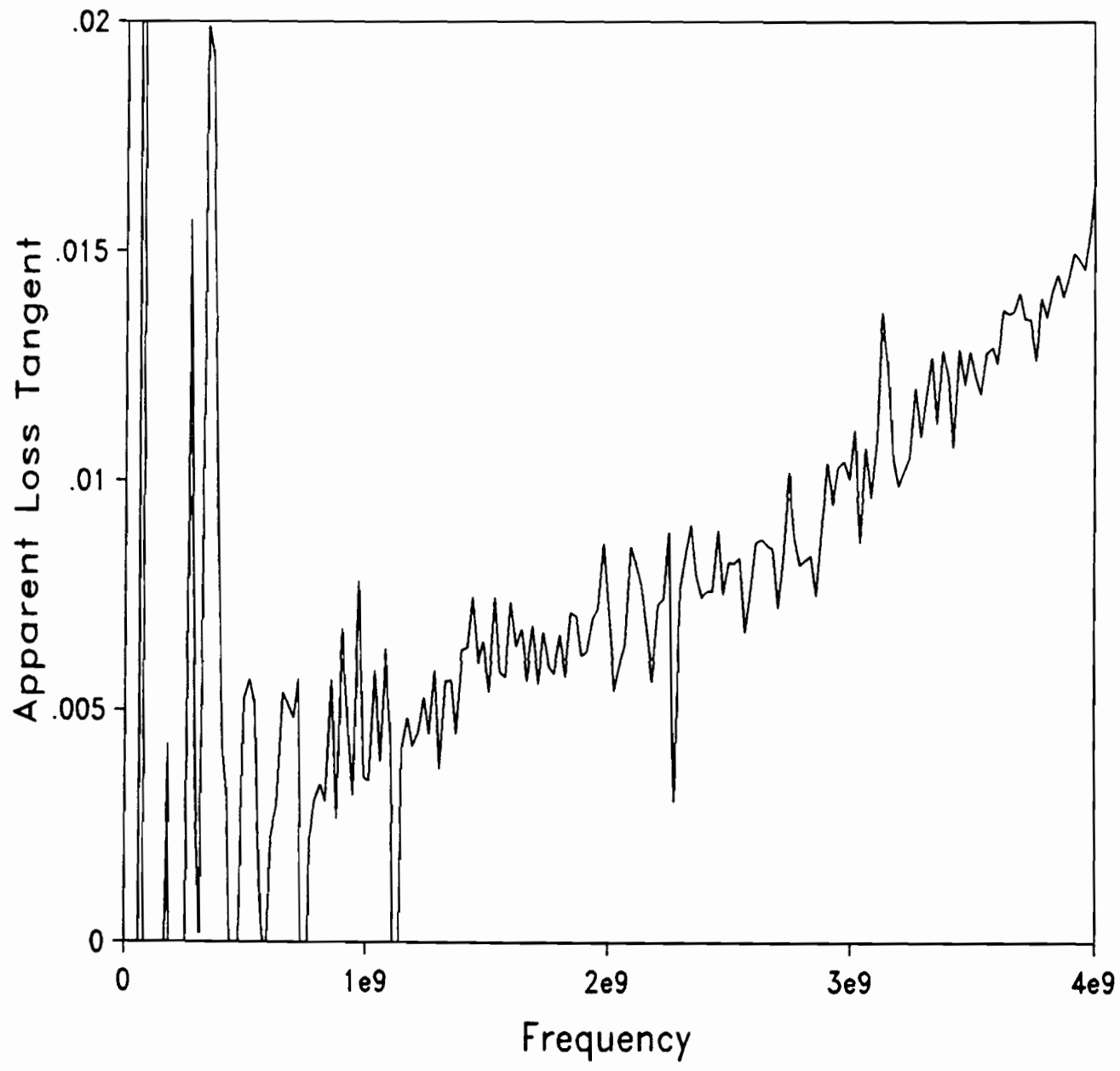

Figure 4.15. Green Tape ${ }^{\mathrm{TM}}$ apparent loss tangent. 


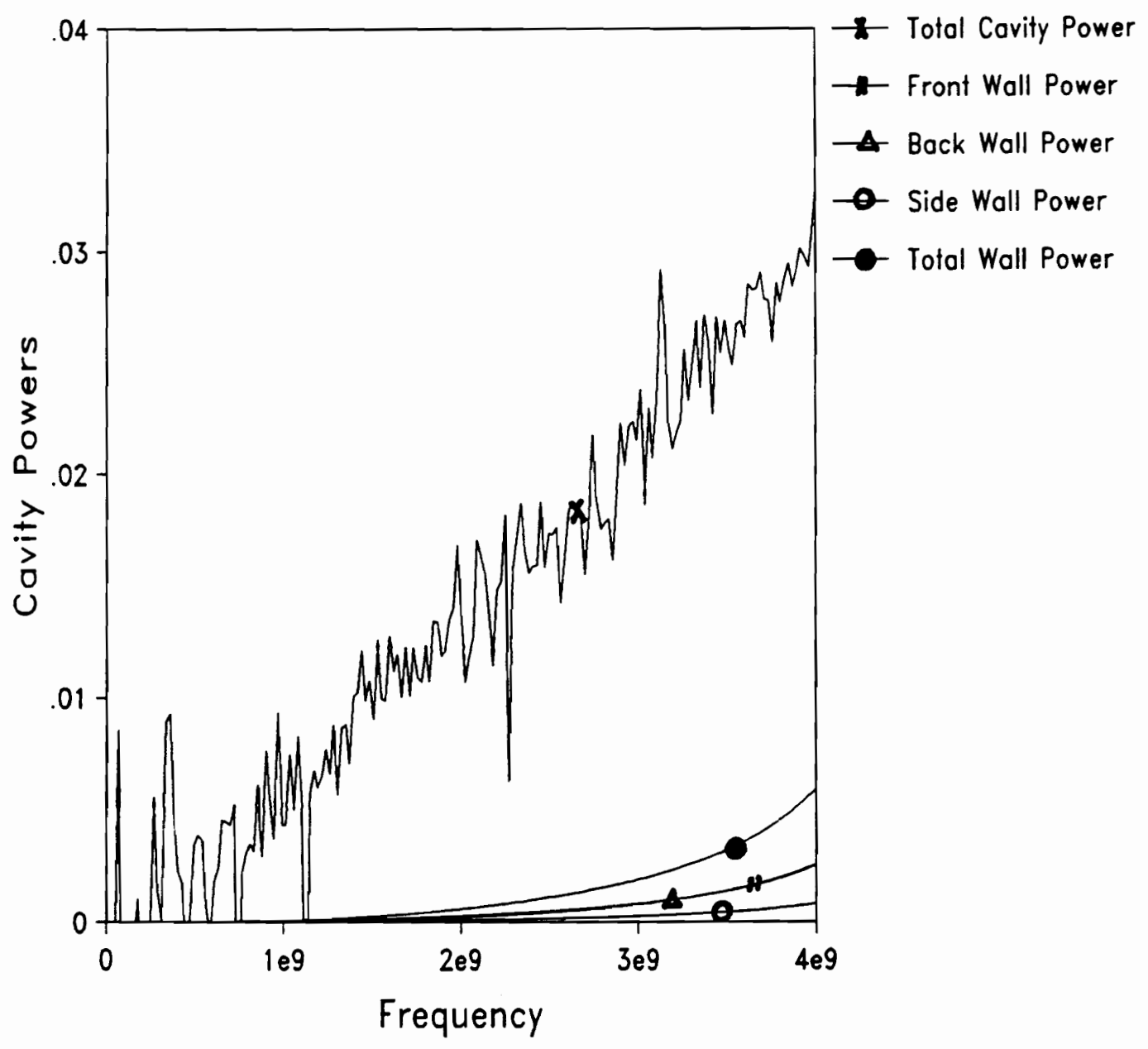

Figure 4.16. Power dissipated in the cavity filled with Green Tape ${ }^{\mathrm{TM}}$. 


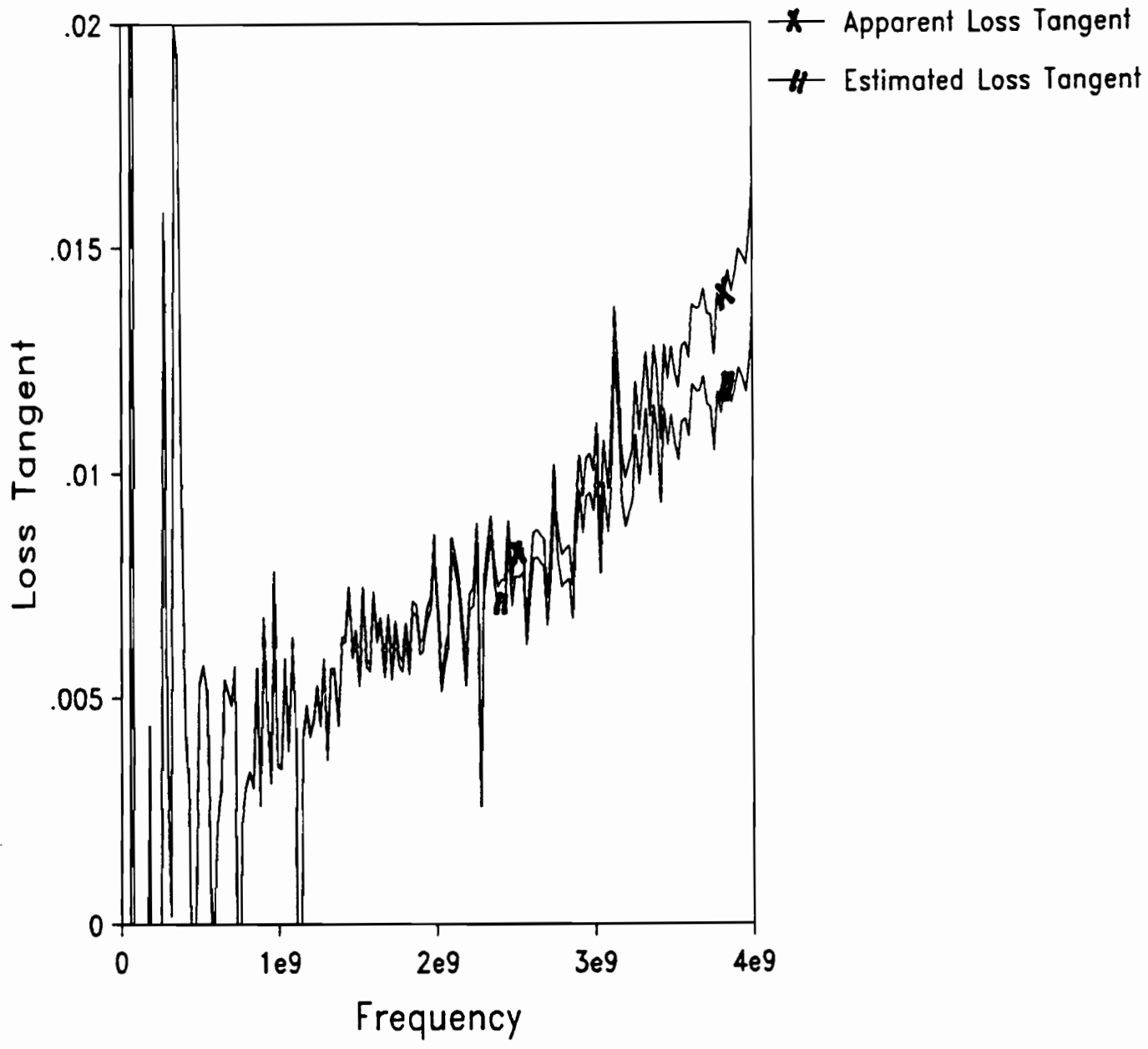

Figure 4.17. Green Tape ${ }^{\mathrm{TM}}$ estimated and apparent loss tangent. 
The dielectric loss tangent estimated after separating the loss of the conductor walls from the apparent loss demonstrates a reasonable behavior and compares well with the published data [Tyler, 1990].

\subsection{SUMMARY}

A simulation problem using typical values for dielectric constant, dielectric loss tangent, cavity dimensions, and conductor conductivity for the cavity walls has been considered to demonstrate the use of this technique including the reflection coefficient measurements and the computation of the dielectric loss tangent.

Measurements were made on two Dupont dielectric materials. The sample preparation, the measurements made with the network analyzer, and the estimated dielectric loss tangents using the perturbation technique were presented and agreed with published data on the materials. 


\section{CHAPTER 5}

\section{ERROR ANALYSIS}

\subsection{INTRODUCTION}

The potential sources of errors for the measurement and perturbation technique may be grouped into three general categories: samples uniformity and geometry, calibration and measurements errors, and errors occurring in the numerical process. In addition to these errors, the technique has additional limitations as discussed in Section 3.3.

The measured data includes the magnitude and phase of the reflection coefficient of a dielectric-filled cavity. The sensitivity of the reflection coefficient to the dielectric constant, the loss tangent, and the sample geometry will be established analytically through the use of a simple model and numerically.

The samples, made to fit the $7 \mathrm{~mm}$ connector of an HP $8510 \mathrm{~B}$ network analyzer, are assumed to be uniform in their geometry and constructed from homogeneous materials. Both the uniformity and homogeneity may be possible sources of error, but the effects on the results are assumed to be negligible and will not be investigated in this work. The samples are made of a dielectric enclosed in a conductor cavity with an aperture adapting to a precision air line. A precise measure of the dielectric thickness is difficult to obtain. The thickness is a potential source of error since this technique is highly sensitive to this dimension. The cavity radius becomes critical when approaching the radial resonant frequency. The error effects of these two dimensions will be investigated. 
Some of the potential sources of errors in the measurement process includes the resolution of the different subsystems, round off errors, and system noise. The HP $8510 \mathrm{~B}$ network analyzer used for the reflection coefficient measurements is a sophisticated equipment with a carefully analyzed system performance. A simulation study, with a random Gaussian noise, based on the instrument uncertainty specifications, added to a simulated reflection coefficient, has been conducted to evaluate the complex permittivity computational behavior in the presence of noise. Related errors result from the effects of system calibration and imprecision of the calibration standards.

The numerical process provides an additional source of error. The truncation of the number of modes in both the transmission line and the cavity regions may lead to a nonconverging solution or one converging to an incorrect solution as discussed in Section 2.5. As a check on the accuracy of the perturbation technique from a purely computational point of view, the technique is used in both a forward and a reverse sense. The forward problem is used to simulate data from which the reverse problem should retrieve the original sample model for different geometrical variations. Also the perturbation technique assumes knowledge of the cavity wall conductivity resulting in a potential error due to an incorrect estimate of conductivity.

The limitations of the perturbation technique come from the assumptions made in the development of the technique. The frequency range limits will be discussed. 
Some error definitions used in this chapter include

Relative error in $x=\frac{\text { error in } x}{x}$,

Percentage error in $x=\frac{\text { error in } x}{x} \times 100$, and

Sensitivity of $x$ to $y=\frac{\text { error in } x}{\text { error in } y} \times 100$.

\subsection{MEASUREMENT SENSITIVITY}

The measured data includes the magnitude and phase of the reflection coefficient of a dielectric-filled cavity. The unperturbed analysis relates the measured reflection coefficient to the complex permittivity of the dielectric. The sensitivity of the reflection coefficient to the dielectric complex permittivity and the sample geometry is considered analytically and checked numerically.

For thin dielectric structures, The dielectric-filled cavity structure may be approximated as a capacitor with an added conductance. This problem is modeled as shown in Fig. 5.1, where the fields are assumed to be concentrated under the transmission line inner conductor and fringing may be neglected. The circuit parameters are given as

$$
C=\frac{\epsilon^{\prime} A}{d}
$$




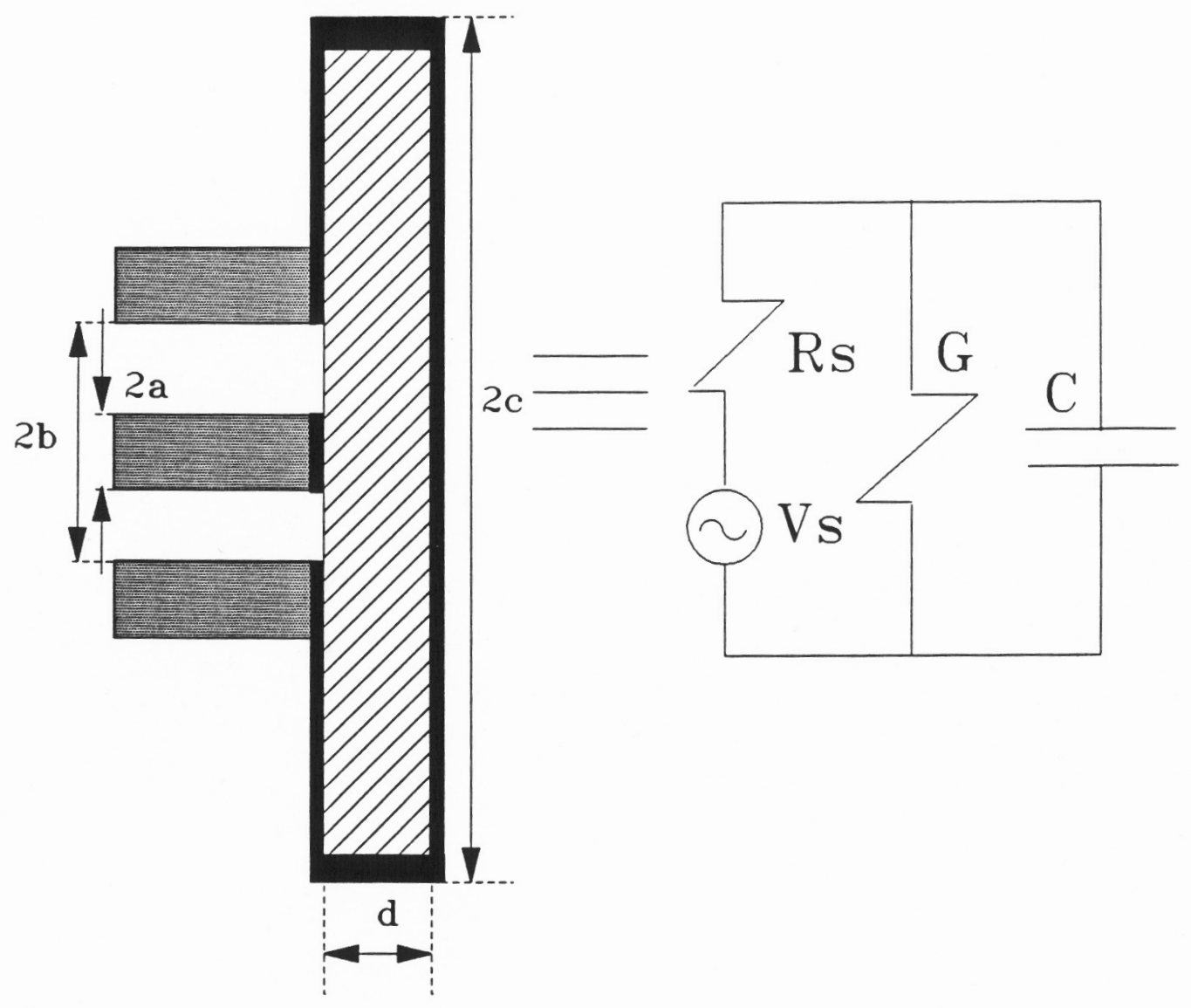

Figure 5.1. Cavity simple model. 
and

$$
G=\frac{\sigma A}{d}=\frac{\omega \epsilon^{\prime \prime} A}{d}
$$

where $A$ is the area of the inner conductor given by $\pi a^{2}$, and $d$ is the dielectric thickness. The source resistance $R_{s}$ is $50 \Omega$ for the matched line. The reflection coefficient, defined as the ratio of the complex amplitudes of the reflected and incident voltage waves at the load, is a complex quantity with a magnitude $\left|s_{11}\right| \leq 1$. This reflection coefficient may be written in terms of the characteristic and load admittances as

$$
\mathrm{s}_{11}=\frac{Y_{o}-Y_{\ell}}{Y_{o}+Y_{\ell}}
$$

where $Y_{0}=0.02$ mhos, and $Y_{\ell}=G+\mathrm{j} \omega C$, or equivalently as

$$
\mathrm{s}_{11}=\frac{f-\epsilon_{r}^{\prime \prime}-\mathrm{j} \epsilon_{r}^{\prime}}{f+\epsilon_{r}^{\prime \prime}+\mathrm{j} \epsilon_{r}^{\prime}}
$$

where $f$ is defined as

$$
f=\frac{Y_{0} d}{\omega A \epsilon_{0}}
$$

For typical values of $d=.02 \mathrm{~cm}$ and $a=0.15 \mathrm{~cm}$, at a $1 \mathrm{GHz}$ frequency, the value of $f$ is about 10 .

Evaluating the magnitude and phase of the reflection coefficient of Eq. (5.4), we obtain

$$
\left|\mathrm{s}_{11}\right|=\frac{f^{2}+\epsilon_{r}^{\prime 2}+\epsilon_{r}^{\prime \prime 2}-2 f \epsilon_{r}^{\prime \prime}}{f^{2}+\epsilon_{r}^{\prime 2}+\epsilon_{r}^{\prime \prime 2}}
$$


and

$$
\mathrm{s}_{11}=\tan ^{-1} \frac{-\epsilon_{r}^{\prime}}{f-\epsilon_{r}^{\prime \prime}}-\tan ^{-1} \frac{\epsilon_{r}^{\prime}}{f+\epsilon_{r}^{\prime \prime}}
$$

Neglecting $\epsilon_{r}^{\prime \prime}$ with respect to $f$ in Eqs. (5.6) and (5.7), the following relations may be obtained

$$
\left|s_{11}\right|=1-\frac{2 f \epsilon_{r}^{\prime \prime}}{f+\epsilon_{r}^{\prime 2}},
$$

and

$$
\mathrm{s}_{11}=-2 \tan ^{-1} \frac{\epsilon_{r}^{\prime}}{f}
$$

From these equations (5.8) and (5.9), it is clear that the magnitude of the reflection coefficient is affected mainly by $\epsilon_{r}^{\prime \prime}$ and the reflection coefficient phase is affected mainly by $\epsilon_{r}^{\prime}$

The sensitivity of the reflection coefficient to the dielectric constant and dielectric loss in the unperturbed analysis was computed numerically by Saed [1987] and showed that the magnitude of the reflection coefficient is indeed sensitive to the dielectric constant while the phase of the reflection coefficient is only sensitive to the dielectric constant.

By substituting $f$ with the expression given by Eq. (5.5) into Eqs. (5.8) and (5.9), one may show that both the magnitude of the reflection coefficient and the phase are proportional to the dielectric thickness $(d)$ as presented numerically in the next section. 


\subsection{GEOMETRICAL ERROR ANALYSIS}

The dielectric samples are typically made by laminating various layers of the dielectric material with a conductor either printed or etched on the front layer to adapt to the network analyzer connector. Estimation of the dielectric and conductor thickness in a sample is complex, requiring separate blank and conductor coated samples. However, the microstructure at the conductor-dielectric interface and the potential nonuniformity in the sample surface make any measured thickness estimate imprecise. Thus variation with geometry is of prime concern.

The first study conducted in this chapter considers the complex permittivity of a dielectric with a thickness $0.020 \mathrm{~cm}$. The reflection coefficient for this material with dielectric constant of 5.0 and an apparent loss tangent as shown in Fig. 5.2 was computed using the forward analysis discussed in Chapter 2. The complex permittivity estimate is obtained from the unperturbed analysis for various thicknesses of the dielectric thicknesses including a $\pm 20 \%$ relative error. The resultant dielectric constants and loss tangents are shown in Figs. 5.3 and 5.4. Relative errors in both the dielectric constant and dielectric loss tangent with respect to a relative error in the dielectric thickness are computed and shown in Fig. 5.5. At $1 \mathrm{GHz}$ frequency, the sensitivity of the dielectric constant to the dielectric thickness is $85 \%$, while the sensitivity of the dielectric loss tangent to the dielectric thickness is $50 \%$. Thus excessive precision must be given in estimating the dielectric thickness.

The next consideration deals with the radial dimension of the cavity region. The samples must have a radius of $0.7458 \mathrm{~cm}$ in order to adapt to the $7 \mathrm{~mm}$ measuring equipment connector. If a sample has a smaller radius, it still fits into the network analyzer connector, but may create an error in the computation of the 


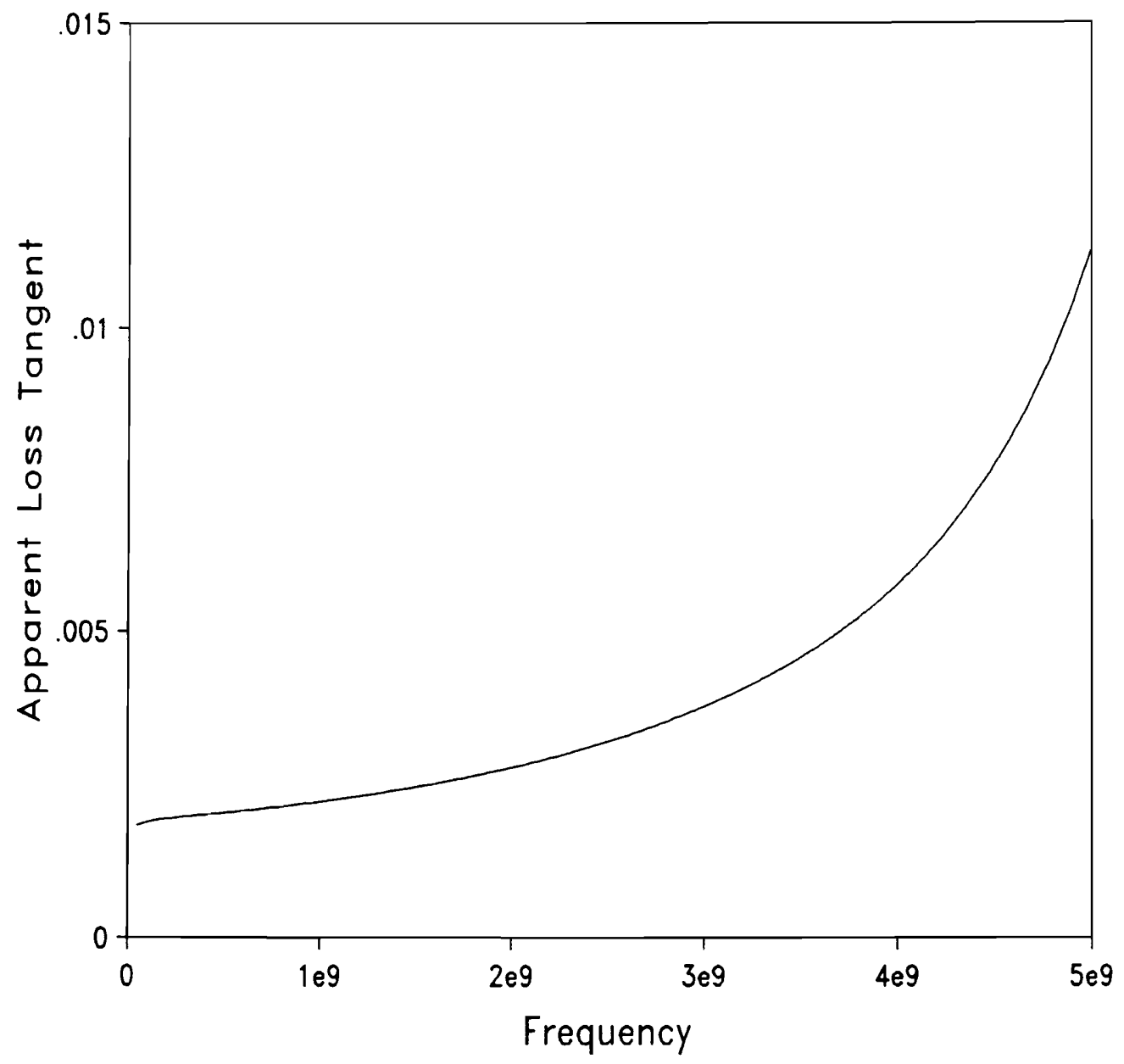

Figure 5.2. Apparent dielectric loss tangent for the simulated problem with $\epsilon_{r}^{\prime}=5.0, d=.02 \mathrm{~cm}, \sigma_{\mathrm{F}}=\sigma_{\mathrm{B}}=6 \times 10^{5} \mathrm{~S} / \mathrm{cm}$, and $\sigma_{\mathrm{E}}=2 \times 10^{5} \mathrm{~S} / \mathrm{cm}$. 


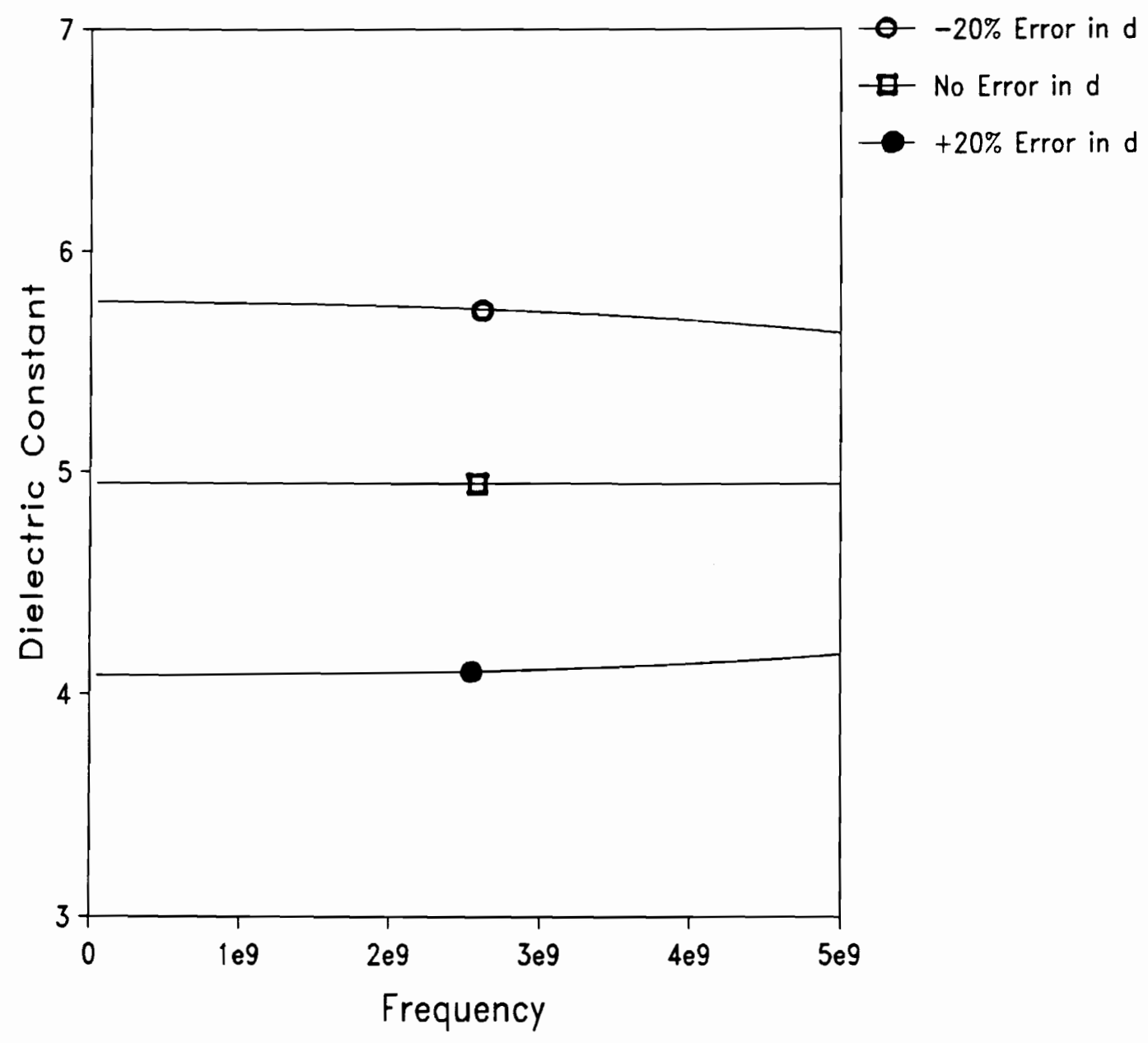

Figure 5.3. Effect of a $\pm 20 \%$ error in the dielectric thickness $d$ on the dielectric constant $\epsilon_{r}^{\prime}$. 


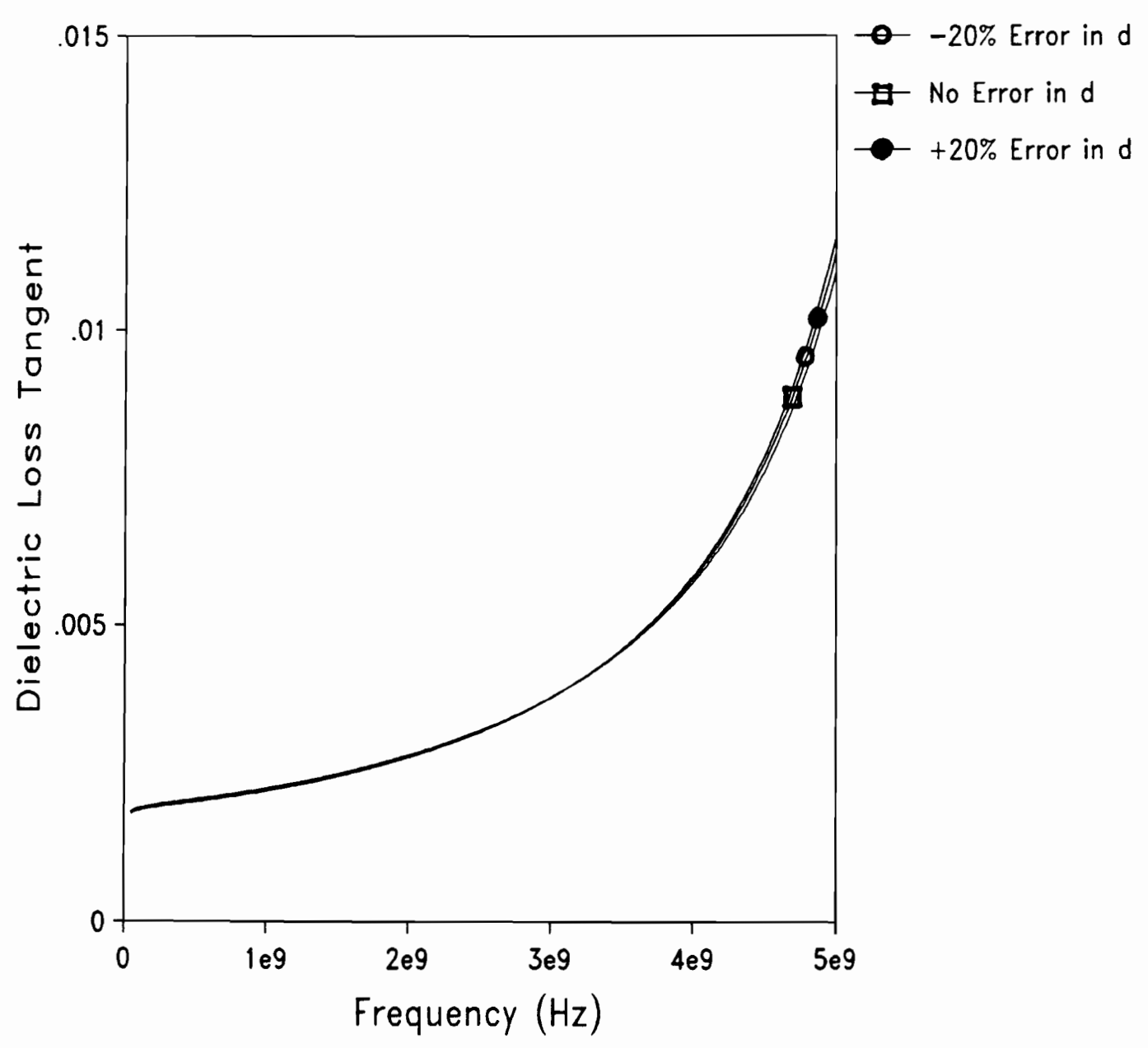

Figure 5.4. Effect of a $\pm 20 \%$ error in the dielectric thickness $d$ on the dielectric loss tangent. 


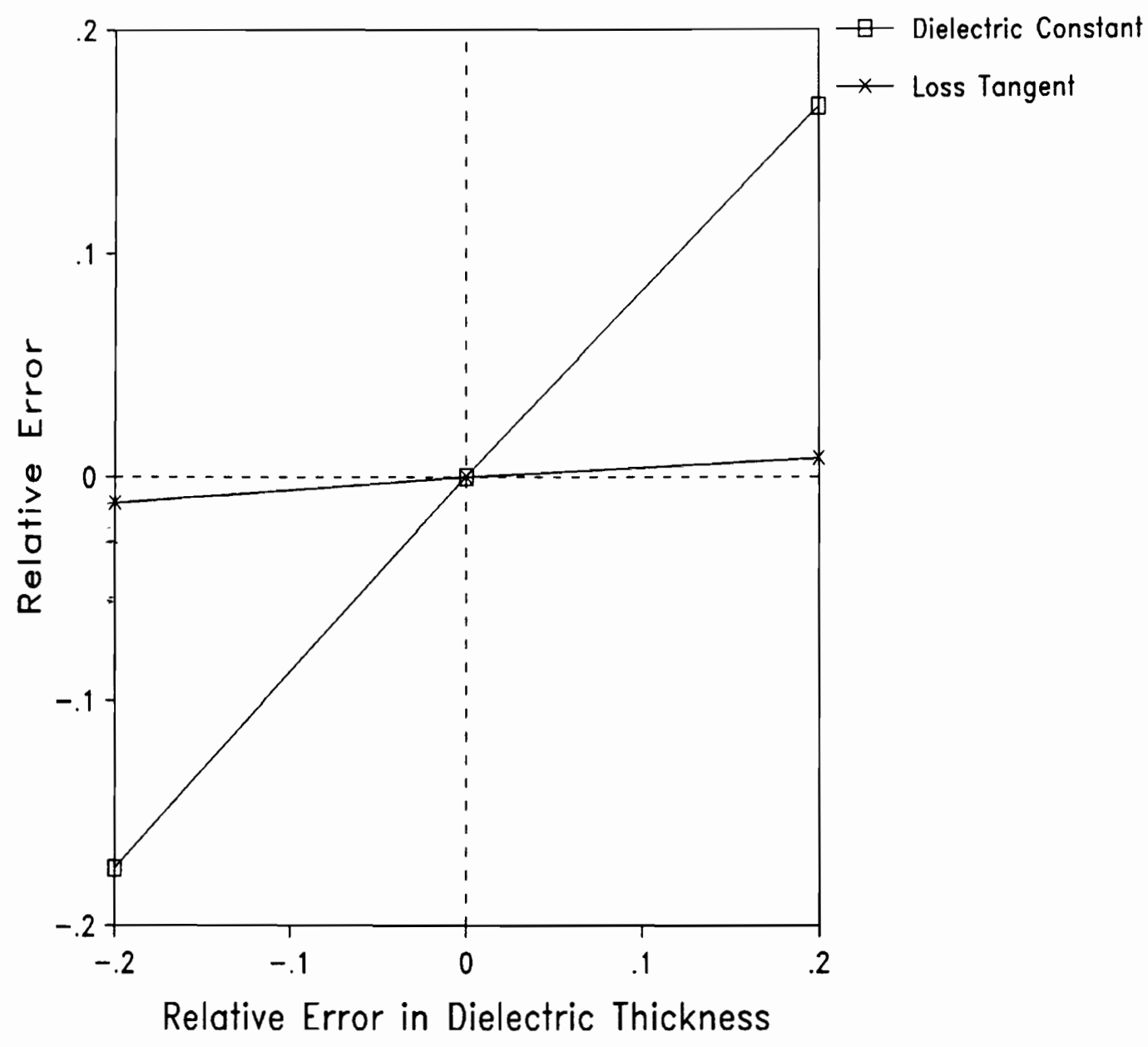

Figure 5.5. Sensitivity of the dielectric constant and the loss tangent the dielectric thickness $d$ at $1 \mathrm{GHz}$ frequency. 
complex permittivity near resonance. This error is not expected to be large since the fields at the cavity edges are minimal except near resonance. The complex permittivity will be estimated using a cavity radius with a $-5 \%$ and a $-10 \%$ error relative to the radius used to simulate the reflection coefficient. The dielectric constants and loss tangents are shown in Figs. 5.6 and 5.7. At $1 \mathrm{GHz}$ frequency, the sensitivity of the dielectric constant and loss tangent to the cavity radius does not exceed $4 \%$ as shown in Fig. 5.8. Only a negative estimate in the radius has been considered due to the physical limitation of the sample holder formed by the connector structure.

\subsection{MEASUREMENT ERROR ANALYSIS}

The reflection coefficient of the dielectric-filled cavity is measured using an HP 8510B network analyzer. According to the specifications of this instrument, there is an uncertainty of 0.04 for the reflection coefficient magnitude and 3 degrees of uncertainty in the phase for a reflection coefficient of unity through $8 \mathrm{GHz}$. Gaussian random noise with these basic specifications was added to the simulated reflection coefficient to simulate realistic measured data. The computed dielectric constant and loss tangent for this problem with the added random noise to the reflection coefficient are shown in Figs. 5.9 and 5.10. These results have the same general behavior as the computed results from actual measured data using the HP 8510B network analyzer as the apparent loss tangents shown in Figs. 4.10 and 4.15 .

\subsection{PERTURBATION TECHNIQUE ERROR ANALYSIS}

The numerical process in the perturbation technique used to separate the 


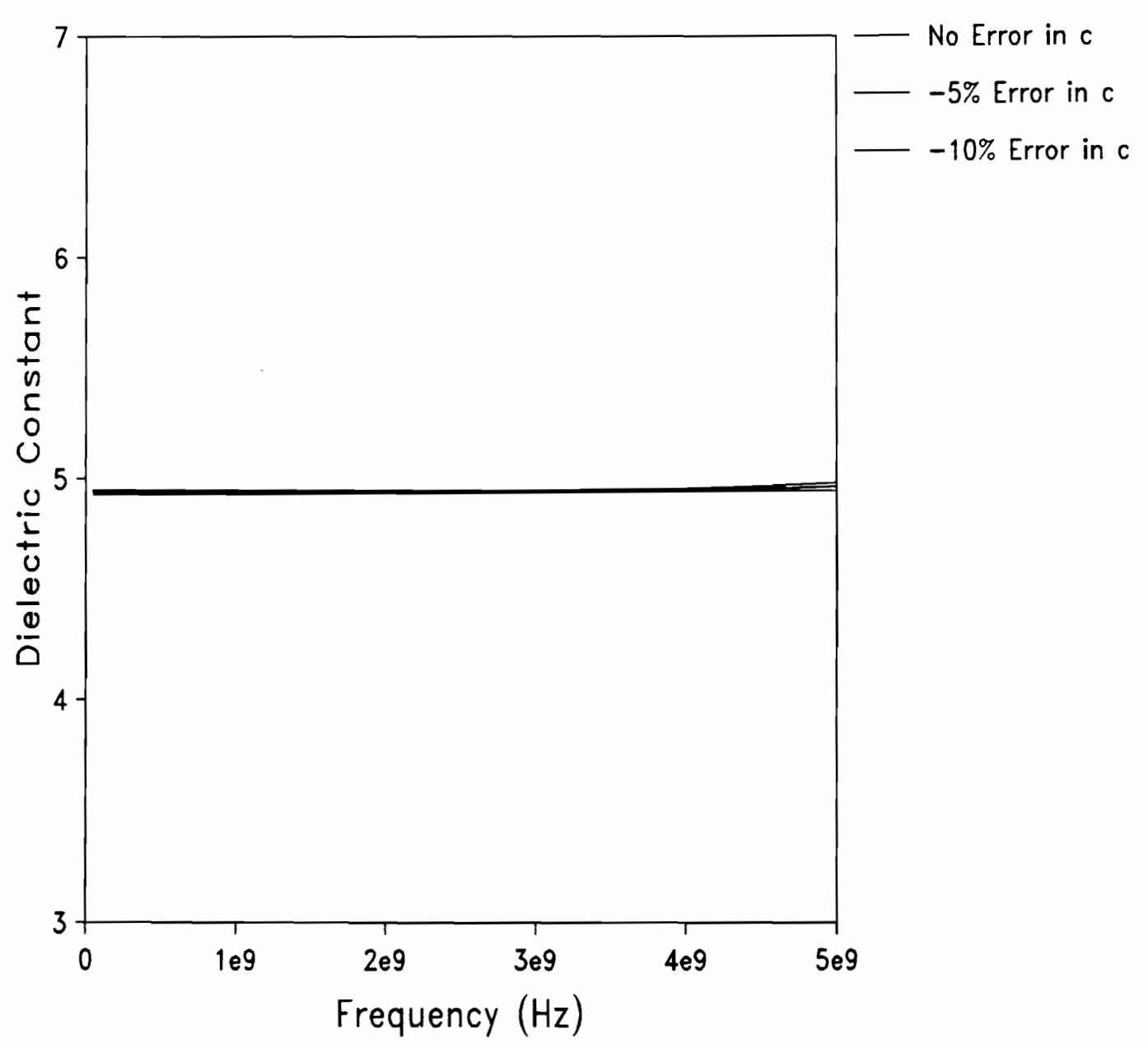

Figure 5.6. Effect of a $-5 \%$ and a $-10 \%$ error in the cavity radius $c$ on the dielectric constant $\epsilon_{r}^{\prime}$. 


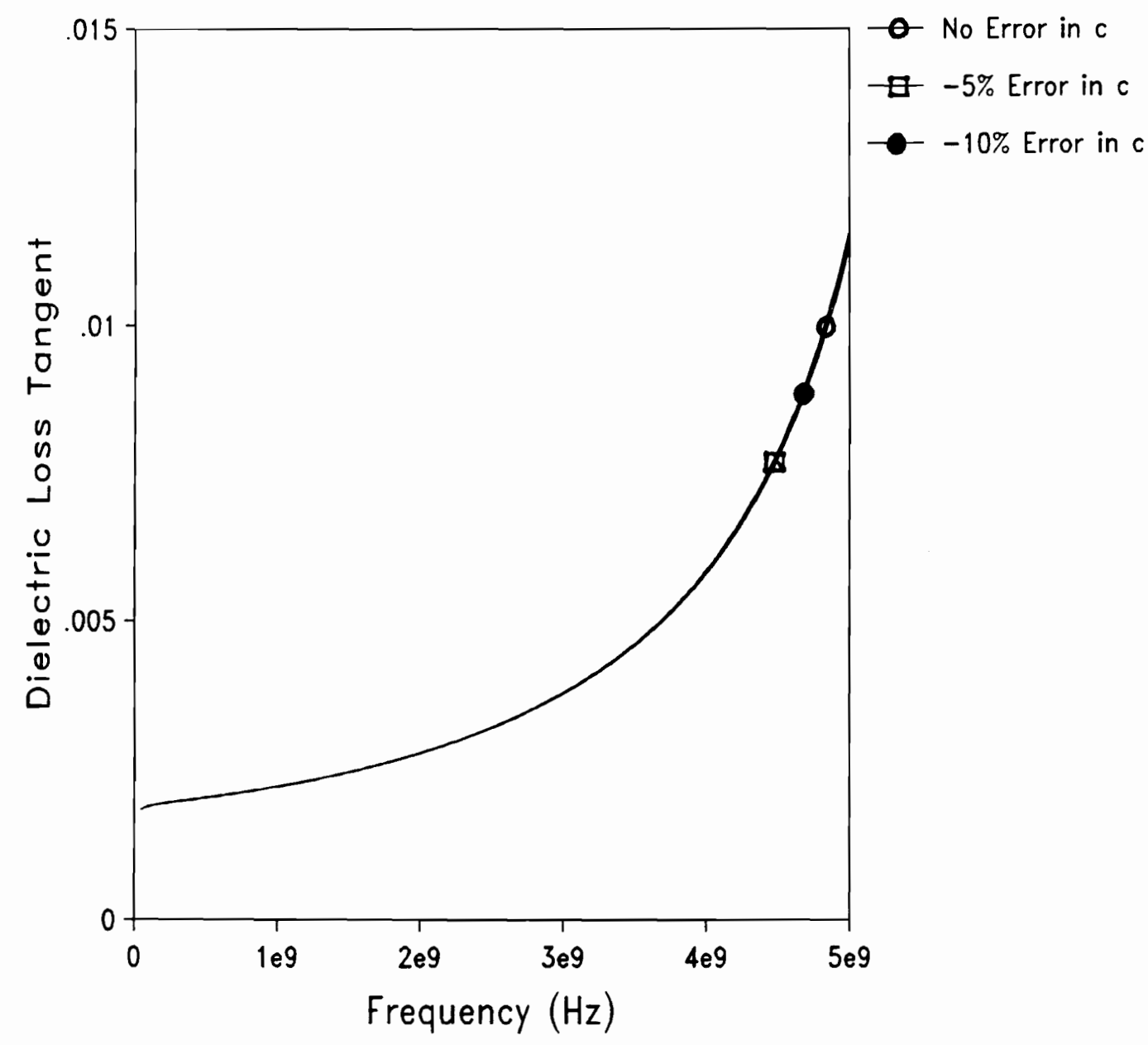

Figure 5.7. Effect of a $-5 \%$ and a $-10 \%$ error in the cavity radius $c$ on the dielectric loss tangent. 


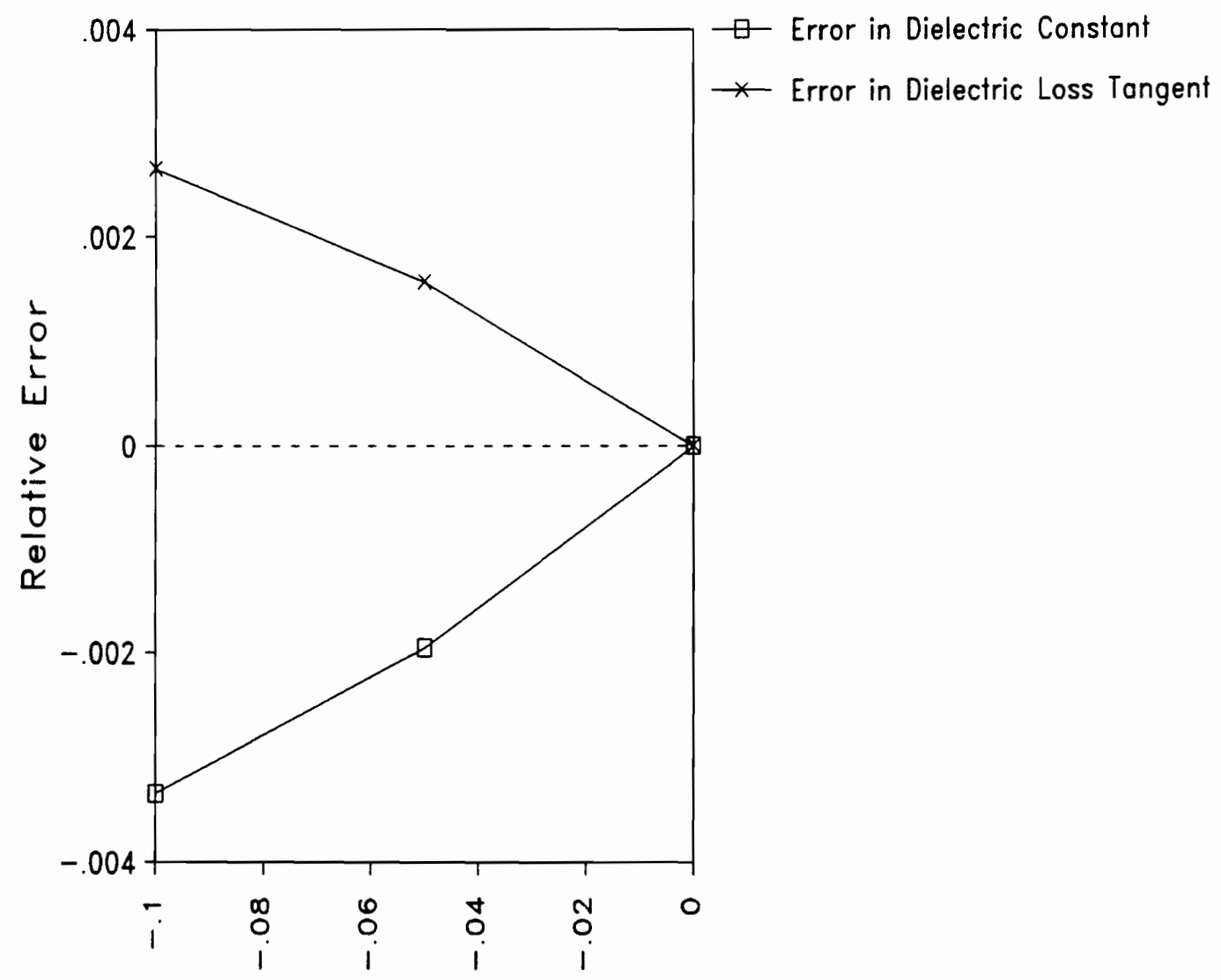

Relative Error in cavity radius

Figure 5.8. Sensitivity of the dielectric constant and the loss tangent to the cavity radius $c$ at $1 \mathrm{GHz}$ frequency. 


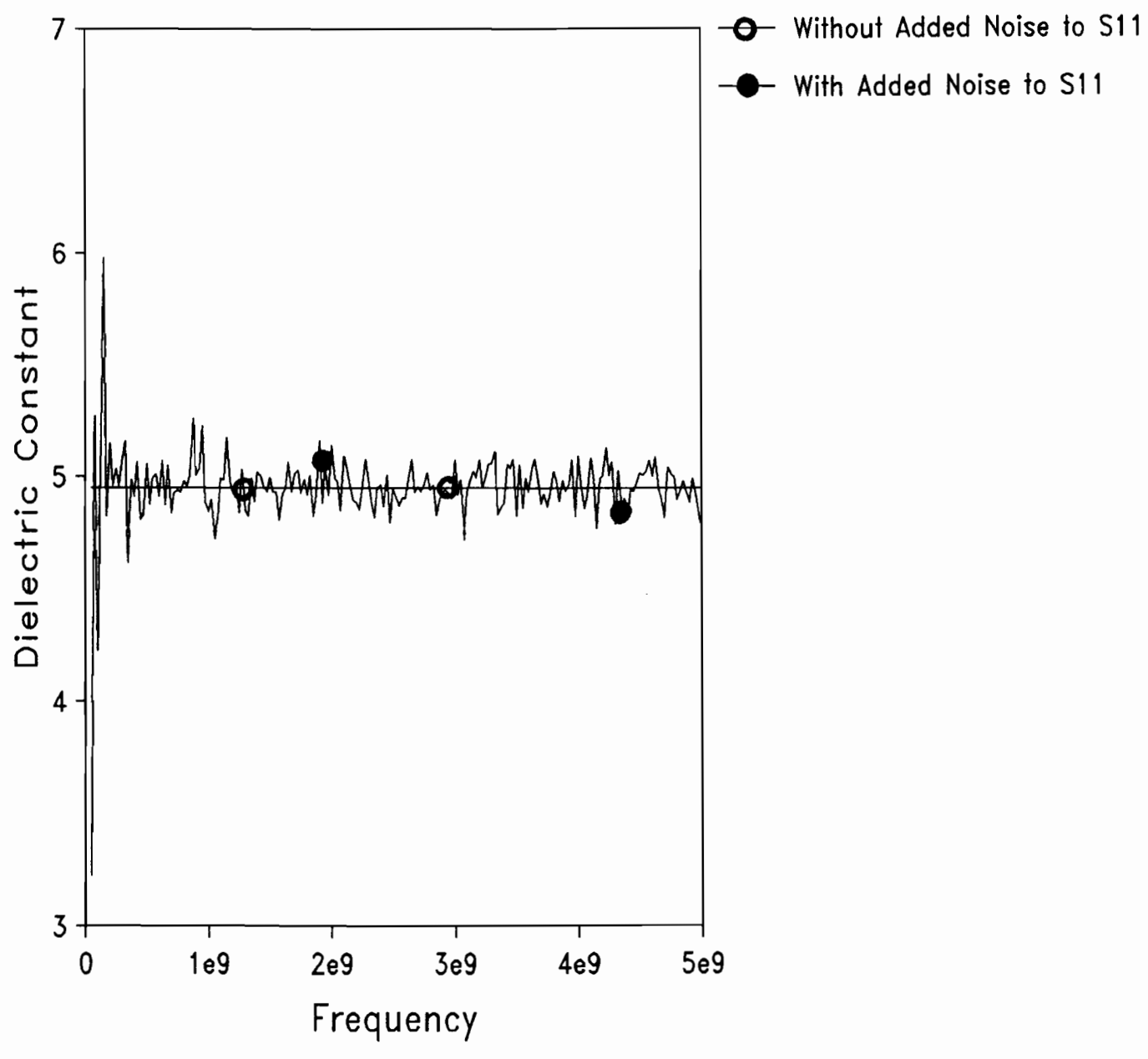

Figure 5.9. Effect of random noise on the dielectric constant $\epsilon_{r}^{\prime}$. 


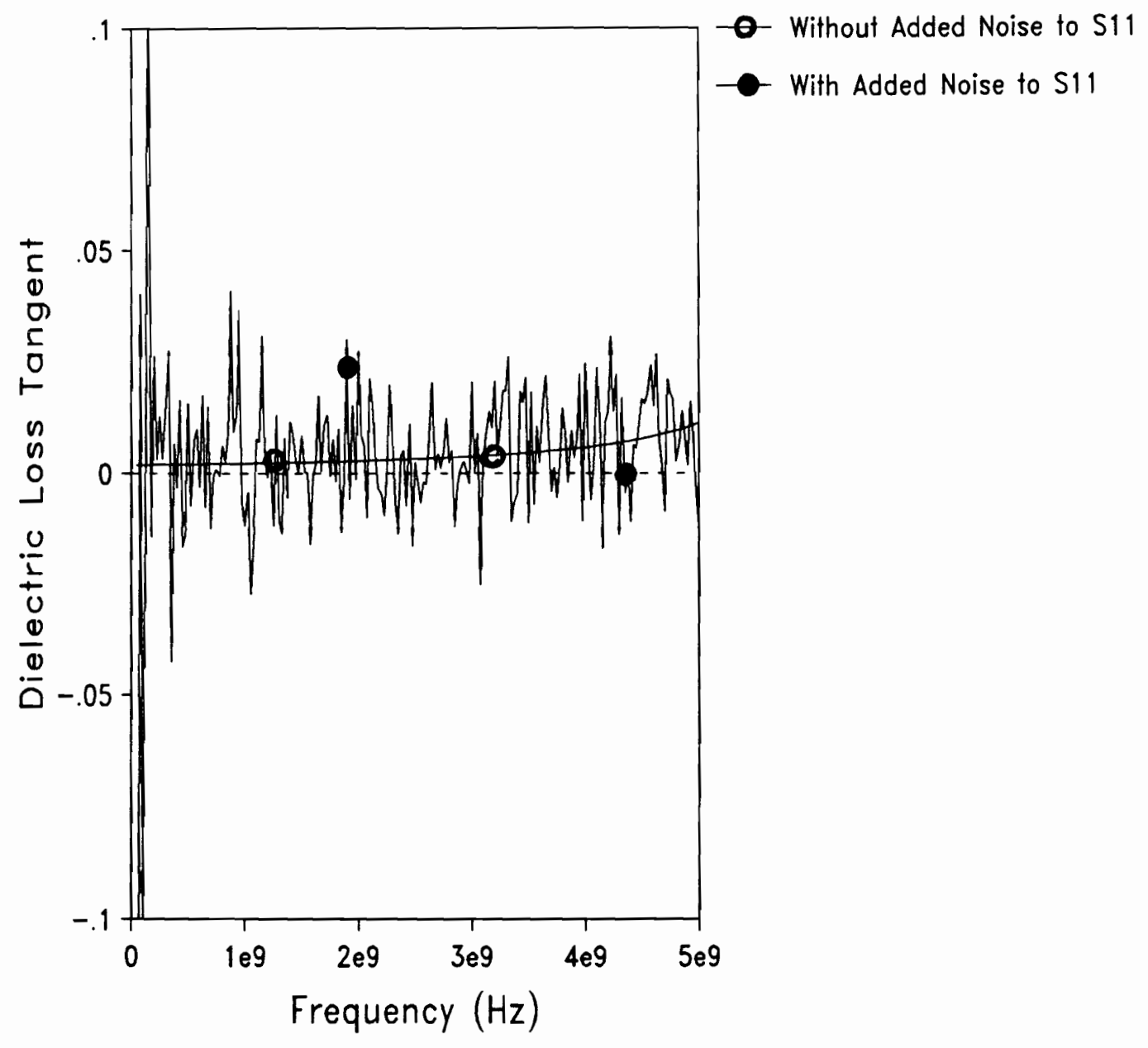

Figure 5.10. Effect of random noise on the dielectric loss tangent. 
conductor loss of the cavity walls from the total loss is the last potential source of error to be considered. To check this numerical error, the perturbation technique is used in a forward sense to compute the power dissipated in a simulated problem with an $\epsilon_{r}^{\prime}$ of 5 , an $\epsilon_{r}^{\prime \prime}$ of 0.01 , a thickness of $0.02 \mathrm{~cm}$, and a conductor conductivity of $6 \times 10^{5} \mathrm{~S} / \mathrm{cm}$. This conductor loss is then added to the dielectric loss of 0.01 to give the apparent loss tangent as in Fig. 5.2. Using the perturbation technique one more time in a reverse sense to compute the dielectric loss of 0.01 . The percentage relative error in the estimated loss tangent is minimal, as shown in Fig. 5.11. The relationship between the error in the dielectric loss estimate and the absolute thickness of a dielectric sample is considered. The forward analysis was used to determine the reflection coefficient for three thicknesses $(d=0.02 \mathrm{~cm}, 0.01 \mathrm{~cm}$ and $0.005 \mathrm{~cm})$. Perturbation analysis was then used to estimate the dielectric loss tangent for each of the cases and results were compared to the initial values of the dielectric loss tangent. The percentage error in the estimated dielectric loss tangent increases sharply with deceasing the thickness of the dielectric as seen from Figs. 5.11, 512, and 5.13. These results indicate a limitation on the perturbation technique. For an expected error of less than $0.1 \%$ in the estimation of the dielectric loss, the dielectric thickness must be at least $0.01 \mathrm{~cm}$ for low dielectric constants over the frequency range of interest. This limitation will vary slightly for different dielectric constants and frequencies. The general recommendation is to use samples as thick as possible to guarantee small numerical error.

To use the perturbation technique in order to compute the power loss in the cavity walls and separate it from the total cavity loss, the conductivity of the conductor material must also be known. To study the effect of this conductivity on the estimated dielectric loss, a simulation has been created for a dielectric constant of 5.0 and a dielectric loss of 0.01 with an added conductor loss for a conductor 


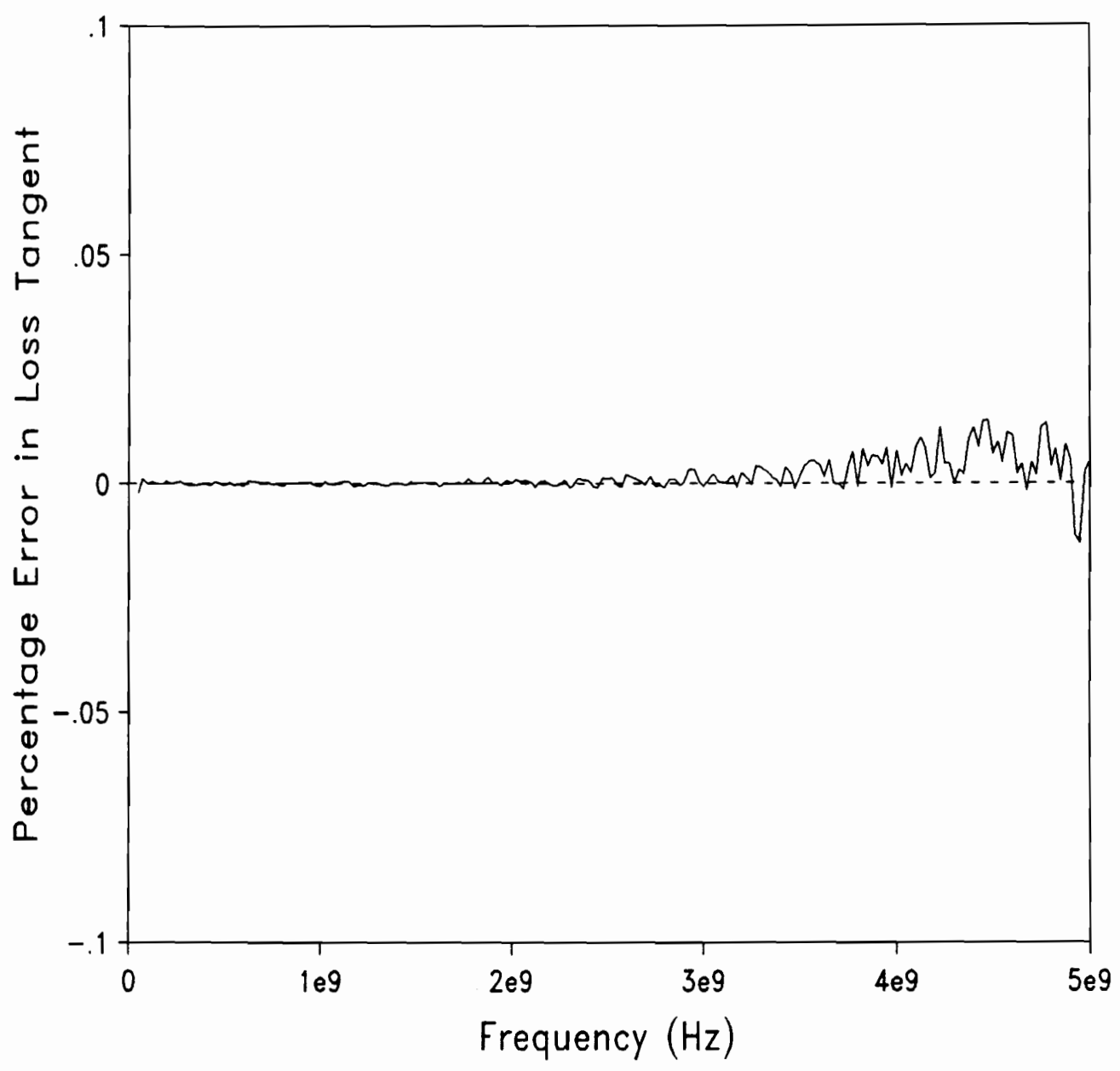

Figure 5.11 Relative numerical error in the estimated dielectric loss tangent for the simulated problem with $d=.02 \mathrm{~cm}$. 


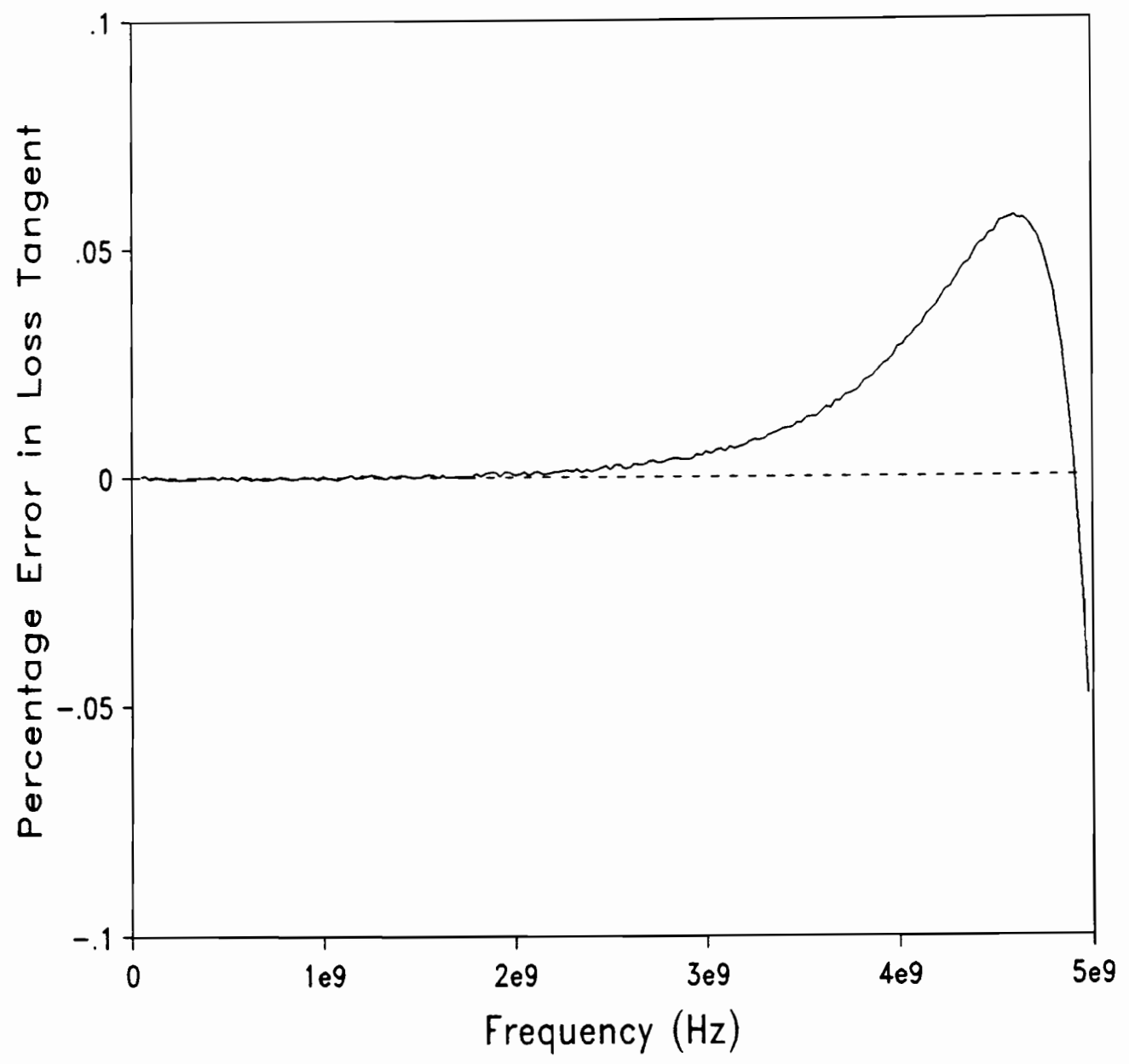

Figure 5.12 Relative numerical error in the estimated dielectric loss tangent for the simulated problem with $d=.01 \mathrm{~cm}$. 


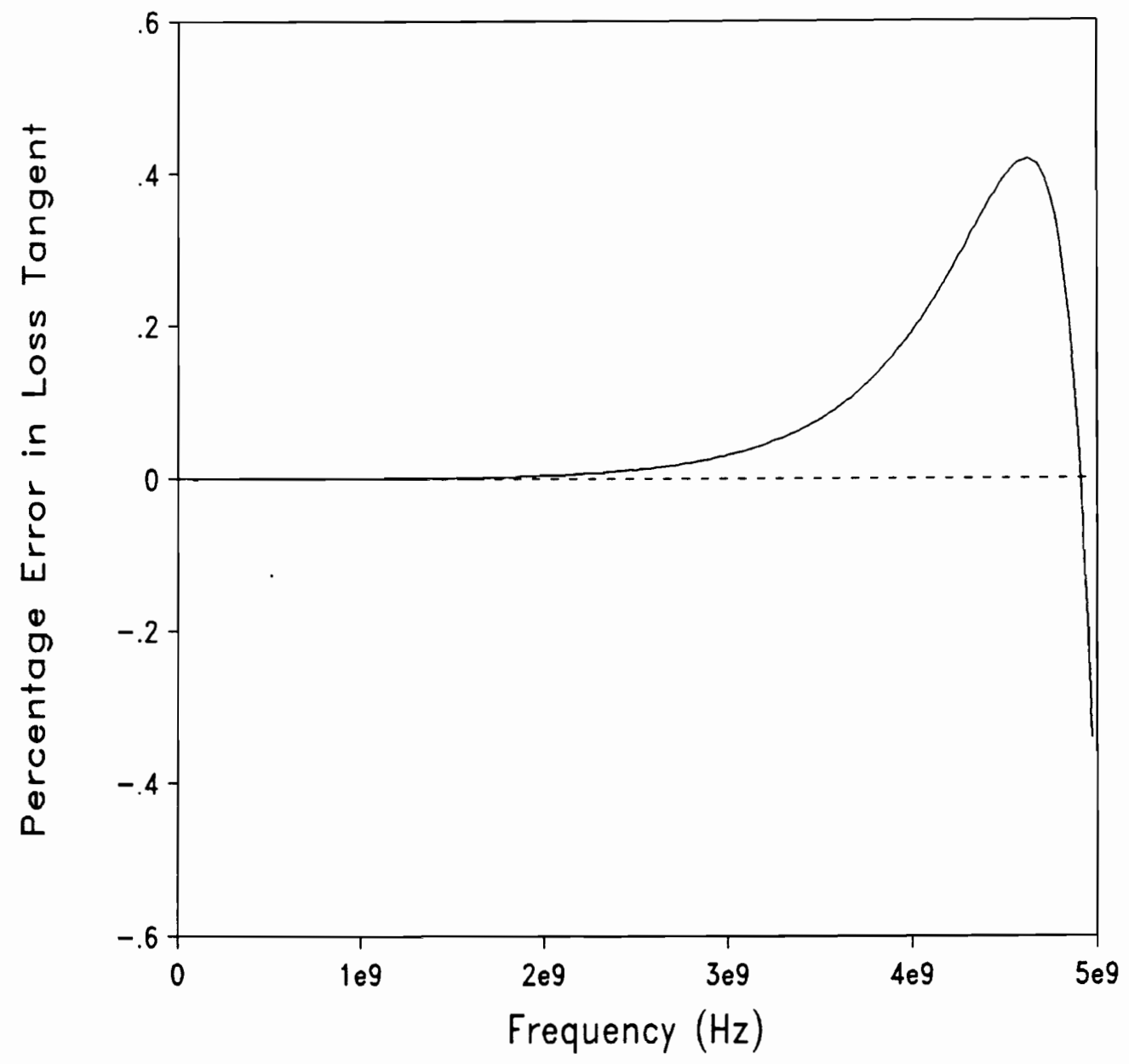

Figure 5.13 Relative numerical error in the estimated dielectric loss tangent for the simulated problem with $d=.005 \mathrm{~cm}$. 
with a conductivity of $6 \times 10^{5} \mathrm{~S} / \mathrm{cm}$, giving the apparent loss tangent shown in Fig. 5.2. The perturbation technique was used to estimate the dielectric loss tangent of the simulated problem for different assumed values of conductor conductivity. Fig. 5.14 shows the apparent dielectric loss tangent and the estimated loss tangents with various assumed conductivities. The assumed conductivities included the original value of $6 \times 10^{5} \mathrm{~S} / \mathrm{cm}$ for the front and back walls of the cavity and $2 \times 10^{5} \mathrm{~S} / \mathrm{cm}$ for the side walls of the cavity and two additional set of values with $\pm 10 \%$ error. The relative error of the loss tangent is a function of the frequency as seen from Fig. 5.15. At a $3 \mathrm{GHz}$ frequency the relative error in the estimated dielectric loss versus the error in the conductor conductivity is shown in Fig. 5.16. At this frequency, the sensitivity of the perturbation technique to the estimation of the conductivity is $40 \%$. This source of error puts a limitation on the use of the technique in the absence of a good estimate of the conductor conductivity. If not known accurately, a higher estimate of the conductivity is then recommended since it will give an upper bound on the dielectric loss tangent. This sensitivity may be reduced by the use of a thicker dielectric sample as suggested in the previous study.

\subsection{PERTURBATION TECHNIQUE LIMITATIONS}

Assumptions were made in Chapter 3 when developing the perturbation approach. These assumptions are now conditions to be satisfied in order to use the developed technique. The first condition is that the conductor walls must be constructed from high conductivity conductors such that $\sigma \gg \omega \epsilon_{0}$. This puts a higher limit on the frequency to be used. For conductivity. of $1 \times 10^{7} \mathrm{~S} / \mathrm{m}$, this upper limit is about $1 \times 10^{16} \mathrm{~Hz}$, which is well above the frequency range of interest. 


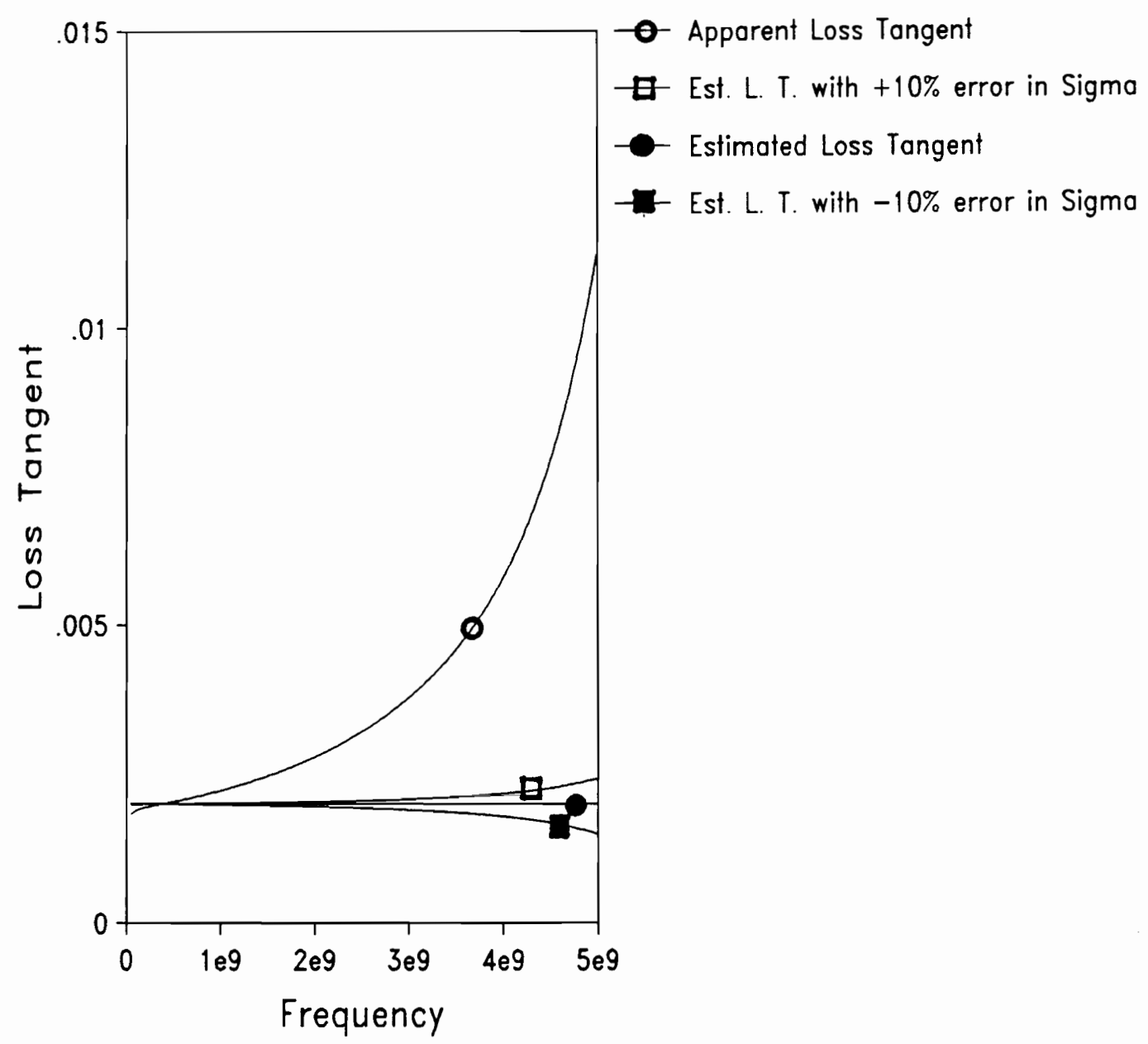

Figure 5.14. Apparent dielectric loss tangent for the simulated problem with $\epsilon_{r}^{\prime}=5.0, d=.02 \mathrm{~cm}, \quad \sigma_{\mathrm{F}}=\sigma_{\mathrm{B}}=6 \times 10^{5}$ $\mathrm{S} / \mathrm{cm}$, and $\sigma_{\mathrm{E}}=2 \times 10^{5} \mathrm{~S} / \mathrm{cm}$ along with the estimated loss tangent with and without a $\pm 10 \%$ error in the conductor conductivity. 


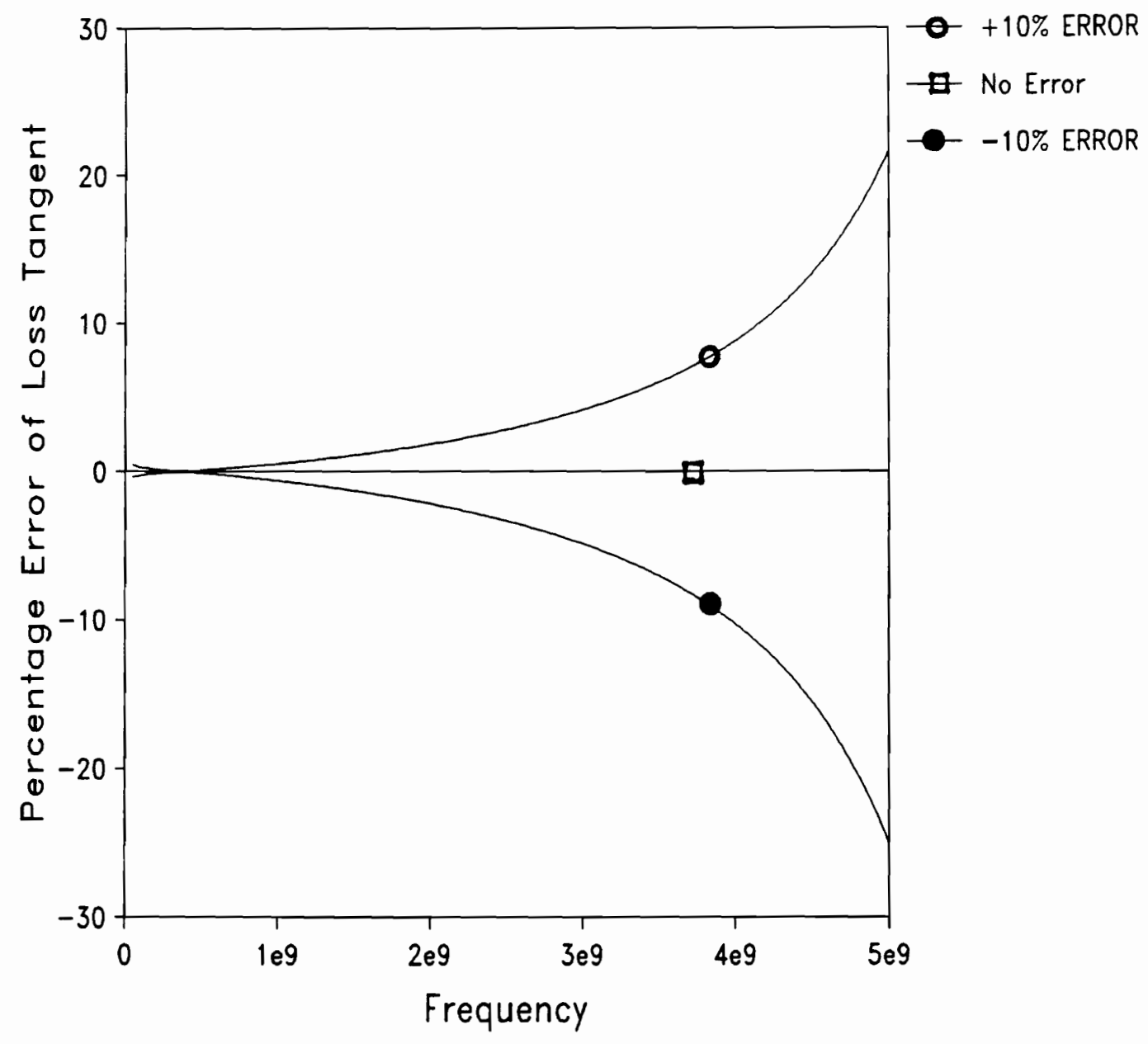

Figure 5.15. Relative percentage error in the dielectric loss tangent for the simulated problem with and without a $\pm 10 \%$ error in the conductor conductivity. 


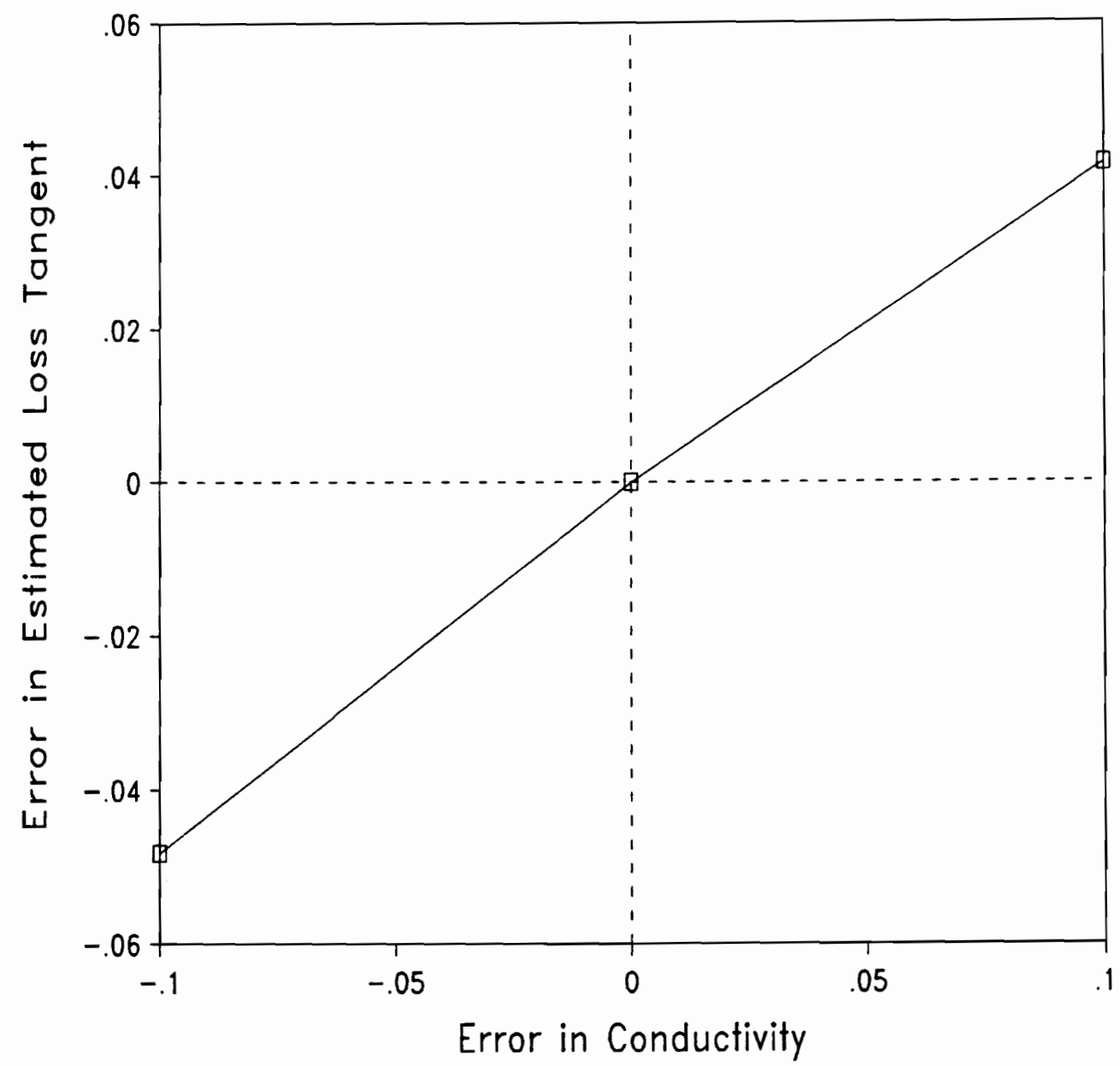

Figure 5.16. Sensitivity of the estimated dielectric loss tangent to the conductor conductivity. 
The second condition is that the conductor thickness must be larger than the skin effect such that $e^{-2 d / \delta} \ll 1$, or $2 d \gg \delta$, where $\delta=\sqrt{2 / \omega \mu_{0} \sigma}$. For conductivity of $1 \times 10^{7} \mathrm{~S} / \mathrm{m}$, and conductor thickness of $0.2 \mathrm{mil}$, the lower limit on the frequency is about $4 \mathrm{kHz}$. This lower limit on the frequency is well below the range of interest.

\subsection{SUMMARY}

Potential sources of error in the measurement and perturbation technique have been discussed in this chapter. The unperturbed cavity problem has been represented by a simple model for thin cavity structures. From the model, the sensitivity of the reflection coefficient to the dielectric characteristics and the cavity geometry was considered analytically. The sources of error were grouped into three general categories. The first group is related to the sample geometry, where two dimensions have been considered: the dielectric thickness and the cavity radius. The next category includes possible sources of error related to the measurements. The effect of the random noise generated by the measuring equipment was considered. The last source of errors was the numerical process. The considered errors included those generated in estimating the dielectric loss tangent using the perturbation technique, their relation to the dielectric thickness, and the effects of an error in estimating the conductor conductivity needed to be known for the use of the perturbation technique. 


\section{CHAPTER 6}

\section{CONCLUSION}

The wideband dielectric-filled cavity technique, developed by Saed [1987], estimates the dielectric constant and the loss tangent of the material within a cavity from measured reflection and transmission coefficients of the cavity structure with known geometrical dimensions. Based on measurements for some low-loss materials with known properties, the dielectric constant was satisfactorily estimated while the loss tangent was typically overestimated, providing an upper bound on the loss tangent. The overestimation was attributed to the fact that the conductor walls of the cavity structure are not ideal (infinite conductivity) as assumed in the cavity analysis. Thus, the estimated loss tangent included the conductor loss. The goal of this research was to account for the conductor loss in order to obtain a better estimate of the dielectric loss.

In order to separate the conductor loss from the total cavity loss, a perturbation approach was used. The unperturbed problem was the cavity structure with assumed perfectly conducting walls, while the perturbed problem included the effect of imperfectly conducting cavity walls. For high conductivity walls, the fields in the cavity are only slightly perturbed from the unperturbed case. The unperturbed magnetic fields at the walls are used to approximate the surface currents at the walls. The power loss in the cavity walls are computed from these perturbed currents. The total loss in the cavity was computed in the unperturbed problem from the magnitude of the measured reflection coefficient. Subtracting the power loss in the cavity walls from the total power loss in the 
cavity, the power loss in the low-loss dielectric was determined. With the ratio of the power loss in the dielectric and the total power loss in the cavity, the loss tangent of the dielectric under test was evaluated from the apparent or effective loss tangent computed with the unperturbed analysis. As a result of this perturbation technique, improved accuracy in the dielectric loss was obtained.

The unperturbed analysis was developed in this work using a Dyadic Green's function formulation. This basic Green's form was developed for two general regions in order to determine the equivalent aperture magnetic current between the two regions. This solution form has been applied to the cavity structure under consideration.

The solution of the unperturbed problem was studied with respect to convergence to an asymptotic value of reflection coefficient. A ratio of the numbers of expansion terms in the Green's functions for the two regions was found to be related to the geometrical dimensions of the two regions. A minimum number of terms in the two regions, related by that ratio, was determined in order to give a reasonable convergence. To verify, as well as to demonstrate, the use of the perturbation technique, two Dupont dielectric materials were characterized using the technique, giving results comparable to published results obtained by other techniques.

Recommendations for use of the perturbation technique were made based on error analysis done of the technique, including sample geometry, frequency measurements and numerical processing. Some of the recommendations include the use of thick dielectric samples to reduce the numerical error. It was also recommended to use an overestimate of the conductor conductivity, when an accurate estimate is not available, to obtain an upper bound on the dielectric loss 
instead of underestimating the value.

Limitations also exist in the assumptions necessary for the formulation of the perturbational application to the specific problem of the wideband dielectricfilled cavity. The interface between the dielectric and the conductor walls was assumed to be smooth and uniform. The thickness of the conductor walls was also assumed to be larger than the skin depth. The skin depth puts a lower limit on the frequency range over which this technique is expected to provide improvement successfully. Below this frequency, the results obtained applying this technique may be questionable, but should have only provided minor changes to the results.

This technique was proven to be successful and may be used by commercial industries to characterize the dielectric materials used in hybrid integrated circuits and multi-layer structure technology. Possible future work may include the expansion of the work done in this research to the transmission cavity structure developed by Saed [1987]. 


\section{APPENDIX}

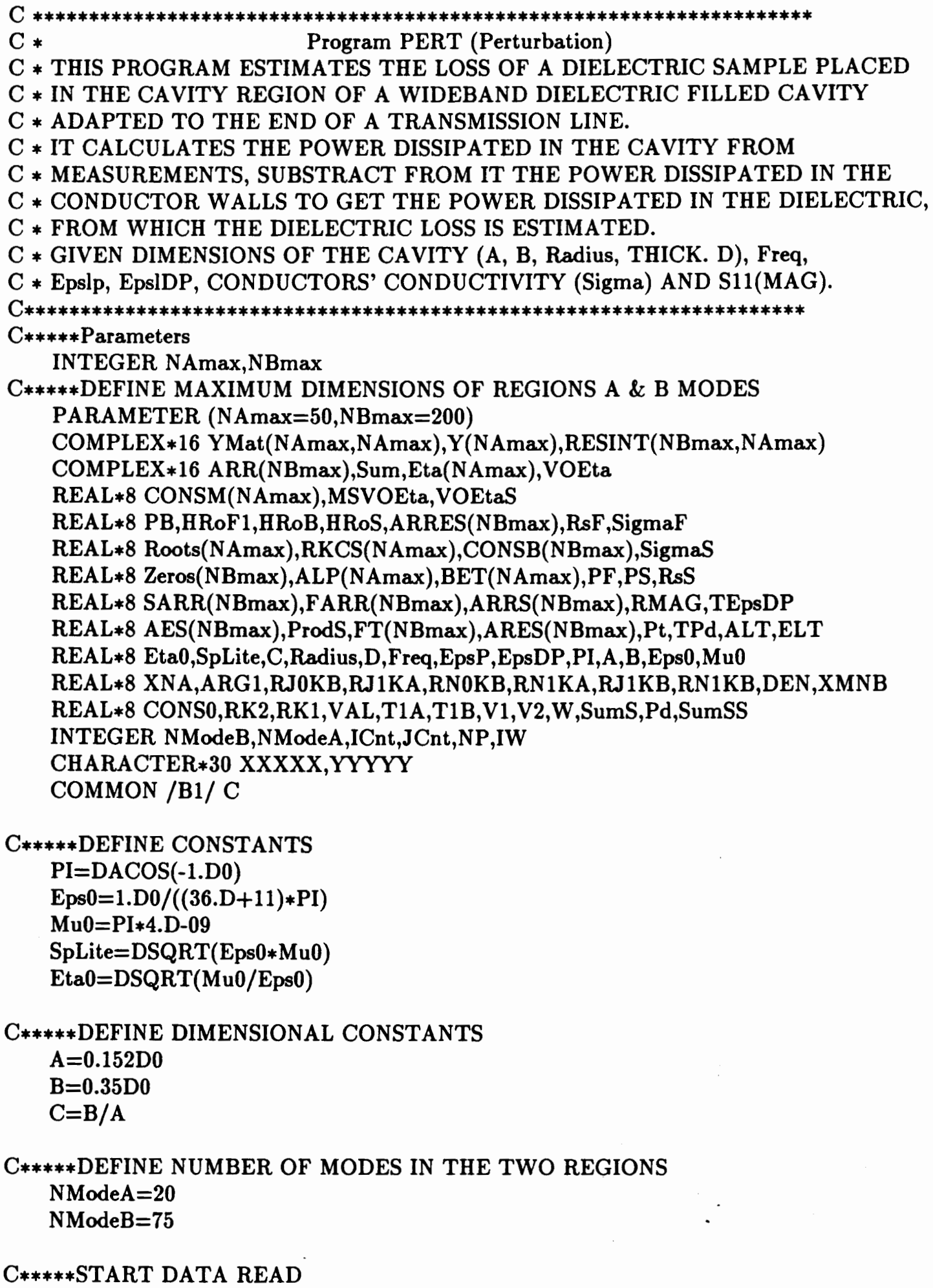


WRITE $(*, *)$ 'ENTER INPUT FILE NAME FOR FREQ,rmag,EpsP AND EpsDP '

$\operatorname{READ}(*, 1) \operatorname{XXXXX}$

1 FORMAT(A30)

OPEN $(4, F I L E=X X X X X)$

WRITE $(*, *)$ 'ENTER OUTPUT FILE NAME FOR DISSIPATED POWER'

$\operatorname{READ}(*, 6)$ YYYYY

6 FORMAT(A30)

OPEN(3,FILE=YYYYY)

WRITE(*,*)'ENTER THE RADIUS (IN CM) OF THE SAMPLE HOLDER'

$\operatorname{READ}(*, *)$ Radius

WRITE $(*, *)$ 'ENTER THE SAMPLE THICKNESS (IN CM)'

$\operatorname{READ}(*, *) \mathrm{D}$

WRITE $(*, *)$ 'ENTER THE NUMBER OF DATA POINTS'

$\operatorname{READ}(*, *) \mathrm{NP}$

WRITE $(*, *)$ 'ENTER THE FRONT \& BACK CONDUCTOR CONDUCTIVITY IN S/CM'

$\operatorname{READ}(*, *)$ SigmaF

WRITE $(*, *)$ 'ENTER THE SIDE CONDUCTOR CONDUCTIVITY IN S/CM'

$\operatorname{READ}(*, *)$ SigmaS

C*****READ THE Roots OF THE BESSEL-NEUMANN COMBINATION AND THE Roots

OF

C***** ZERO ORDER BESSEL FUNCTION OF THE FIRST KIND

DO $99 \mathrm{IW}=1, \mathrm{NP}$

READ $(4, *)$ Freq,RMAG,EpsP,EpsDP

WRITE $(*, *)$ 'FREQUENCY ',Freq

$\mathrm{W}=2 . \mathrm{D} 0 * \mathrm{PI} * \mathrm{Freq}$

YMat $=0$

$\mathrm{Y}=0$

CALL RTS(PI,NModeA,NModeB,Roots,Zeros,NAmax,NBmax)

DO 13 ICnt=1,NModeA

$\operatorname{RKCS}(\mathrm{ICnt})=\operatorname{Roots}(\mathrm{ICnt}) * \operatorname{Roots}(\mathrm{ICn} t) /(\mathrm{A} * \mathrm{~A})$

13 CONTINUE

DO 4 ICnt $=2$, NModeA

XNA $=$ Roots(ICnt-1)

ARG1 $=\mathrm{C} * \mathrm{XNA}$

CALL BESSEL(0,XNA,RJOKB)

CALL BESSEL(1,ARG1,RJ1KA)

CALL NEUM(0,XNA,RNOKB)

CALL NEUM(1,ARG1,RN1KA)

$\mathrm{ALP}(\mathrm{ICnt})=\mathrm{B} *(\mathrm{RJ} 0 \mathrm{~KB} * \mathrm{RN} 1 \mathrm{KA}-\mathrm{RN} 0 \mathrm{~KB} * \mathrm{RJ} 1 \mathrm{KA})$

CALL BESSEL(1,XNA,RJ1KB)

CALL NEUM(1,XNA,RN1KB)

BET(ICnt) $=\mathrm{A} *(\mathrm{RJ} 0 \mathrm{~KB} * \mathrm{RN} 1 \mathrm{~KB}-\mathrm{RN} 0 \mathrm{~KB} * \mathrm{RJ} 1 \mathrm{~KB})$

DEN=DSQRT $($ PI $*($ ALP(ICnt $) * A L P(I C n t)-B E T(I C n t) * B E T(I C n t)))$

4 CONTINUE

CONSM (ICnt $)=1$. Do $/$ DEN

DO 14 ICnt $=1$, NModeB

$\mathrm{XMNB}=\mathrm{Zeros}$ (ICnt)

CALL BESSEL(1,XMNB;FT(ICnt))

CONSB $($ ICnt $)=2 . D 0 *$ DSQRT $(P I) /($ Radius $*$ FT $($ ICnt $))$ 
14 CONTINUE

CONS0 =1.D0/DSQRT(2.D0*PI*DLOG(C))

DO 2 ICnt $=1$, NModeB

RK2=Zeros(ICnt)/Radius

CALL BESSEL(0,RK2*B,FARR(ICnt))

CALL BESSEL(0,RK2*A,SARR(ICnt))

2 CONTINUE

DO 17 ICnt $=1$,NModeB

RK2=Zeros(ICnt)/Radius

RESINT $($ ICnt, 1$)=$ CONS $0 * \operatorname{CONSB}($ ICnt $) *(\operatorname{SARR}($ ICnt $)-F A R R(I C n t)) /$ RK2

DO 17 JCnt=2, NModeA

RK1=Roots(JCnt-1)/A

IF(DABS(RK1-RK2).GT.1.E-08) THEN

$\mathrm{VAL}=\mathrm{CONSM}(\mathrm{JCnt}) * \mathrm{CONSB}(\mathrm{ICnt}) /(\mathrm{RK} 1 * \mathrm{RK} 1-\mathrm{RK} 2 * \mathrm{RK} 2)$

RESINT(ICnt,JCnt) $=$ VAL $*$ RK2

\& $\quad$ (FARR(ICnt)*ALP(JCnt)-SARR(ICnt)*BET(JCnt))

ELSE

CALL BESSEL $(1, \mathrm{RK} 1 * \mathrm{~B}, \mathrm{~T} 1 \mathrm{~B})$

CALL BESSEL $(1, \mathrm{RK} 1 * A, T 1 A)$

$\mathrm{V} 1=\mathrm{B} * \mathrm{~T} 1 \mathrm{~B}-(\mathrm{FARR}$ (ICnt)/RK2)

V2=A*T1A-(SARR(ICnt)/RK2)

RESINT $($ ICnt,JCnt $)=$ CONSM $($ JCnt $) *(\operatorname{CONSB}($ ICnt $) / 2)$

$\&$

ENDIF *(ALP(JCnt)*V1-BET(JCnt)*V2)

17 CONTINUE

C*****EVALUATE THE MATRIX ELEMENTS REPRESENTING THE INCIDENT FIELDS

DO 7 ICnt $=1$, NModeA

$\mathrm{Y}(\mathrm{ICnt})=\mathbf{0 . \mathrm { D } 0}$

7 CONTINUE

$\mathrm{Y}(1)=2 . \mathrm{D} 0 * \mathrm{DSQRT}(2.0) / \mathrm{DSQRT}(\mathrm{Eta} 0)$

C*****EVALUATE THE COEFFICIENTS Y(NModeA) NEEDED TO EVALUATE THE $\mathrm{C} * * * * * \quad$ POWERS IN THE CAVITY

CALL REGA(Eta0,SpLite,W,Eps0,RKCS,NModeA,YMat,NAmax,Eta)

CALL REGB(D,Radius,Mu0,Eps0,W,NModeB,NModeA,

\&Zeros,EpsP,EpsDP,RESINT,YMat,NAmax,NBmax,ARR,ARES,ARRES,ARRS,AES)

CALL GAUSS(NModeA,YMat,Y,NAmax)

C*****INITIALIZATION

ProdS $=0.0$

$\mathrm{HRoB}=0.0$

$\mathrm{HROS}=\mathbf{0 . 0}$

HRoF $1=0.0$

VOEta $=\mathrm{Y}(1) / \mathrm{Eta} 0$

MSVOEta $=$ DREAL $($ VOEta $) *$ DREAL $($ VOEta $)+$ DIMAG(VOEta) $*$ DIMAG(VOEta)

DO $500 \mathrm{JCnt}=2$, NModeA

VOEta $=\mathrm{Y}(\mathrm{Jcnt}) / \mathrm{Eta}(\mathrm{Jcnt}-1)$ 


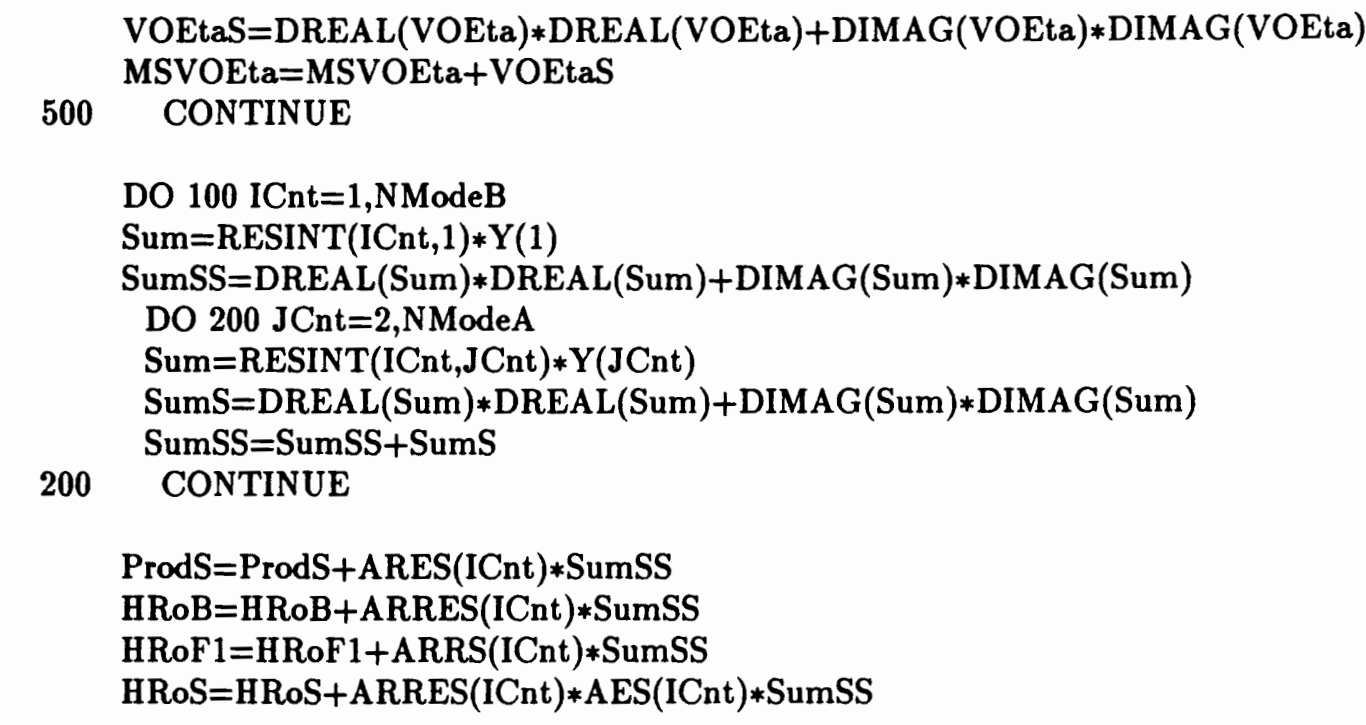

100 CONTINUE

C*****EVALUATE THE POWER ABSORBED BY THE CAVITY

C \& USING S11 MEASUREMENTS

$\mathrm{Pd}=(1.0-(\mathrm{RMAG} * \mathrm{RMAG}))$

C*****EVALUATE THE SURFACE RESISTIVITY OF THE FRONT AND BACK WALLS

$\mathrm{RsF}=2 . \mathrm{E} 0 * \mathrm{PI} * \mathrm{SQRT}$ (Freq*1.E-09/SigmaF)

C*****EVALUATE THE SURFACE RESISTIVITY OF THE SIDE WALL

RsS $=2 . E 0 * P I * S Q R T$ (Freq*1.E-09/SigmaS)

C*****EVALUATING THE POWER DISSIPATED IN THE FRONT WALL

$\mathrm{PF}=(\mathrm{RsF} / 2 . \mathrm{E0}) *($ HRoF $1-\mathrm{MSVOEta})$

C*****EVALUATE THE POWER DISSIPATED IN THE BACK WALL

$\mathrm{PB}=(\mathrm{RsF} / 2 . \mathrm{E} 0) * \mathrm{HRoB}$

C*****EVALUATE THE POWER DISSIPATED IN THE SIDE WALL

PS $=($ RsS $/$ Radius $) *$ HRoS

C*****ADDING UP THE TOTAL POWER LOST IN THE WALLS

$P t=P F+P B+P S$

C*****EVALUATE THE NET POWER DISSIPATED IN THE DIELECTRIC

$\mathbf{T P d}=\mathbf{P d}-\mathbf{P t}$

C*****ESTIMATE THE DIELECTRIC LOSS FACTOR (EpsDP)

$T E p s D P=T P d * E p s D P / P d$

C*****EVALUATE BOTH THE APPARENT AND THE ESTIMATED LOSS TANGENT

ALT $=E p s D P / E p s P$

ELT $=$ TEpsDP $/$ EpsP 
WRITE(3,15) Freq,Pd,PF,PB,PS,Pt,ALT,ELT

15 FORMAT(8(1X,1PE18.8))

99 CONTINUE

STOP

END

$\mathrm{C} * * * * * * * * * * * * * * * * * * * * * * * * * * * * * * * * * * * * * * * * * * * * * * * * * * * * * * * * * * * * * * * * * * * * * * * *$

$\mathrm{C} *$

$\mathrm{C}$ * THE FOLLOWING SUBROUTINE COMPUTES THE ELEMENTS OF THE MATRIX

$C *$ REPRESENTING THE TRANSMISSION LINE REGION

$\mathrm{C} *$

$\mathrm{C} * * * * * * * * * * * * * * * * * * * * * * * * * * * * * * * * * * * * * * * * * * * * * * * * * * * * * * * * * * * * * * * * * * * * * * * *$

SUBROUTINE REGA(Eta0,SpLite,W,Eps0,RKCS,NModeA,YMat,NAmax,Eta)

COMPLEX*16 YMat(NAmax,NAmax),Eta(NAmax),RKMN

INTEGER ICnt,NModeA

REAL $* 8$ RKCS(NAmax),RK,RKS,Eps0,SpLite,W,Eta0

$\mathrm{RK}=\mathrm{W} *$ SpLite

$\mathrm{RKS}=\mathrm{RK} * \mathrm{RK}$

$\operatorname{YMat}(1,1)=1.0 / \mathrm{Eta0}$

DO 1 ICnt $=2$, NModeA

RKMN=CDSQRT(RKS-RKCS(ICnt-1))

$\operatorname{Eta}(\mathrm{ICnt}-1)=\mathrm{RKMN} /(\mathrm{W} * \mathrm{Eps} 0)$

YMat $($ ICnt, ICnt $)=(W *$ Eps0) $/$ RKMN

1 CONTINUE

RETURN

END

$\mathrm{C} * * * * * * * * * * * * * * * * * * * * * * * * * * * * * * * * * * * * * * * * * * * * * * * * * * * * * * * * * * * * * * * * * * * * *$

$\mathrm{C}$ * SUBROUTINE REGB EVALUATES THE MATRIX ELEMENTS REPRESENTING THE

$\mathrm{C}$ * CAVITY REGION. IT ALSO FORMS THE MATRIX AND VECTOR TO BE USED

$\mathrm{C} *$ IN THE GAUSSIAN ELIMINATION ROUTINE (SUBROUTINE GAUSS).

$\mathrm{C} * * * * * * * * * * * * * * * * * * * * * * * * * * * * * * * * * * * * * * * * * * * * * * * * * * * * * * * * * * * * * * * * * * * * * *$

SUBROUTINE REGB(D,Radius,Mu0,Eps0,W,NModeB,NModeA, \&Zeros,EpsP,EpsDP,RESINT,YMat,NAmax,NBmax,ARR,ARES,ARRES,ARRS,AES)

INTEGER NB1

parameter $(\mathrm{NB1}=200)$

COMPLEX $* 16 \mathrm{Jsm}, \mathrm{KzD}$, EpsB,KDSQ,SUMB,ARR(NBmax), ARRE(NB1),

\& YMat(NAmax,NAmax),K,RESINT(NBmax,NAmax)

REAL*8 Zeros(NBmax), Alpha,Beta,MAArg,MBArg,ARRS(NBmax)

REAL $* 8$ D,Radius,Mu0,Eps0,W,EpsP,EpsDP,ARRES(NBmax)

REAL $* 8$ Sn,Snh,ARES(NBmax),AArg,BArg,MSIN,AES(NBmax)

INTEGER ICnt,JCnt,LCnt,NModeB,NModeA,NAmax,NBmax

$\mathrm{Jsm}=\mathrm{DCMPLX}(0 . \mathrm{D} 0,1 . \mathrm{D0})$

EpsB $=$ DCMPLX $($ EpsP, - EpsDP $) * E p s 0$

$\mathrm{KDSQ}=\mathrm{W} * \mathrm{~W} * \mathrm{EpsB} * \mathrm{Mu} 0$ 
DO 1 ICnt $=1$, NModeB

$\mathrm{KzD}=\mathrm{CDSQRT}$ (KDSQ-(Zeros(ICnt)/Radius) $* * 2)$

$\mathrm{K}=\mathrm{KzD} * \mathrm{D}$

$\operatorname{ARR}(I C n t)=J s m * W *(E p s B * \operatorname{CDCOS}(K) /(K z D * \operatorname{CDSIN}(K)))$

C*****EVALUATING THE CLOSED FORM OF THE INTEGRATION

C \& OF THE MAGNITUDE SQUARE OF SIN((KzD(D-Z))

Alpha $=$ REAL $(\mathrm{KzD})$

Beta $=$ IMAG(KzD)

AArg $=2.0 *$ Alpha $* \mathrm{D}$

BArg $=2.0 *$ Beta $*$ D

MAArg $=$ DABS(AArg)

MBArg $=$ DABS(BArg)

IF(MAArg.LT.1.0E-5) THEN

$\mathrm{Sn}=(2.0 *$ Alpha $* \mathrm{D}) /(2.0 *$ Alpha $* \mathrm{D})$

ELSE

$\mathrm{Sn}=(\operatorname{DSIN}(2.0 *$ Alpha $* \mathrm{D})) /(2.0 *$ Alpha $* \mathrm{D})$

ENDIF

IF(MBArg.LT.1.0E-5) THEN

$\operatorname{Snh}=(2.0 *$ Beta $* D) /(2.0 *$ Beta $* D)$

ELSE

$\mathrm{Snh}=(\operatorname{DSINH}(2.0 *$ Beta*D$)) /(2.0 *$ Beta $* \mathrm{D})$

ENDIF

$\operatorname{MSIN}=\operatorname{DREAL}(\operatorname{CDSIN}(\mathrm{K})) * \operatorname{DREAL}(\operatorname{CDSIN}(\mathrm{K}))$

\& +DIMAG(CDSIN(K))*DIMAG(CDSIN(K))

ARES $($ ICnt $)=(($ Snh-Sn $) *(D / 2.0)) /$ MSIN

$\operatorname{AES}(\mathrm{ICnt})=(\mathrm{Snh}+\mathrm{Sn}) *(\mathrm{D} / 2.0)$

C*****COMPUTING ARRE(ICnt) AS ARR(ICnt) WITH $\mathrm{Z}=\mathrm{D}$

$\operatorname{ARRE}(\operatorname{ICnt})=\mathrm{Jsm} * \mathrm{~W} * \operatorname{EpsB} /(\mathrm{KzD} * \operatorname{CDSIN}(\mathrm{KzD} * \mathrm{D}))$

ARRES(ICnt) $=$ DREAL (ARRE(ICnt) $) *$ DREAL(ARRE(ICnt))

$\&$ +DIMAG(ARRE(ICnt))*DIMAG(ARRE(ICnt))

C*****COMPUTING ARRS(ICnt) AS ARR(ICnt) SQUARE WHERE Z=0

ARRS(ICnt) $=$ DREAL(ARR(ICnt))*DREAL (ARR(ICnt))

\& +DIMAG(ARR(ICnt)) $*$ DIMAG(ARR(ICnt))

1 CONTINUE

DO 2 ICnt $=1$, NModeA

DO 2 JCnt $=1$, NModeA

SUMB $=0$. .D0

DO 3 LCnt $=1$, NModeB

SUMB=SUMB+ARR(LCnt)*RESINT(LCnt,ICnt) $*$ RESINT(LCnt,JCnt)

3 CONTINUE

YMat(ICnt,JCnt) $=$ YMat(ICnt,JCnt)-SUMB

2 CONTINUE

RETURN

END 


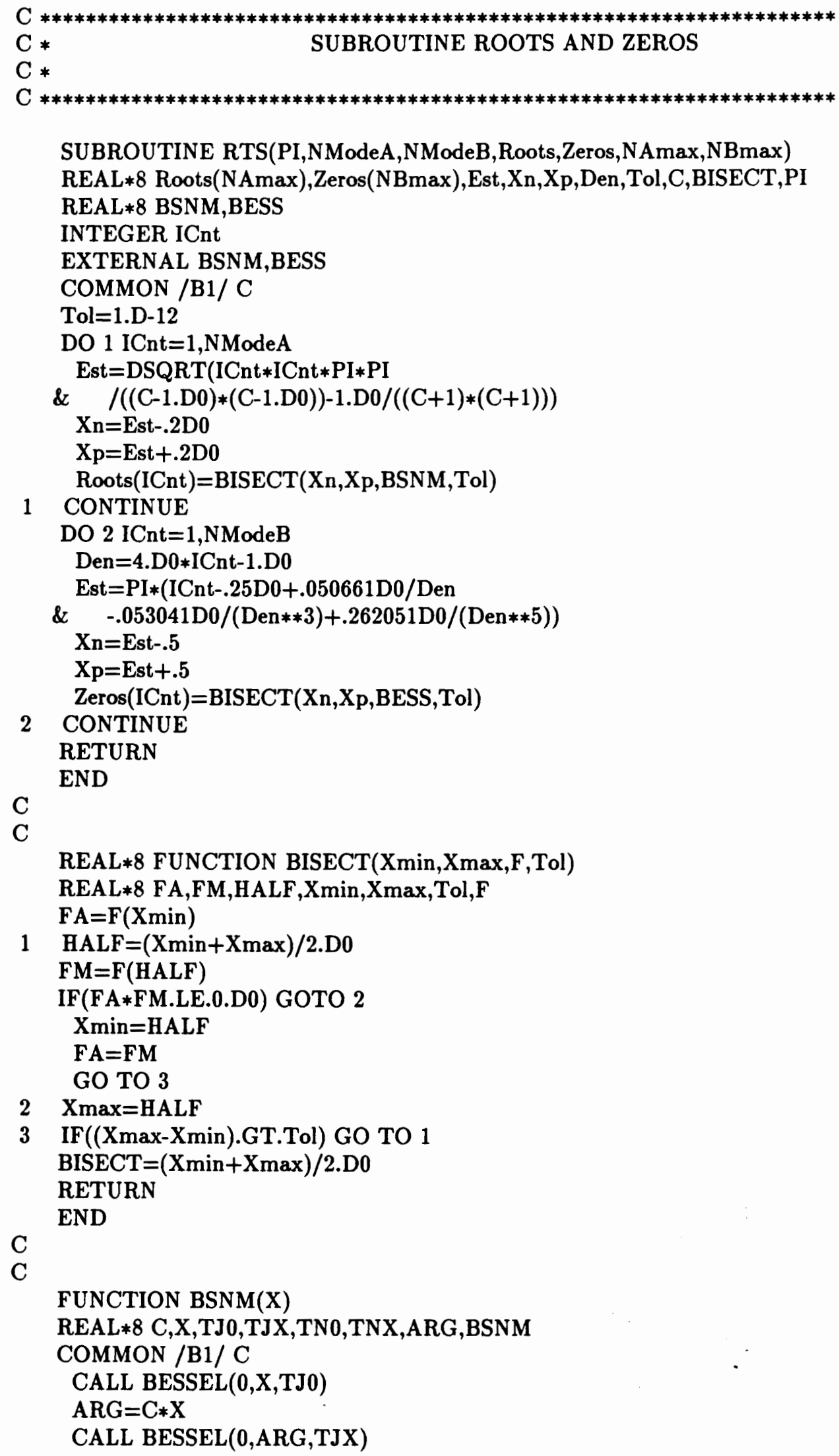




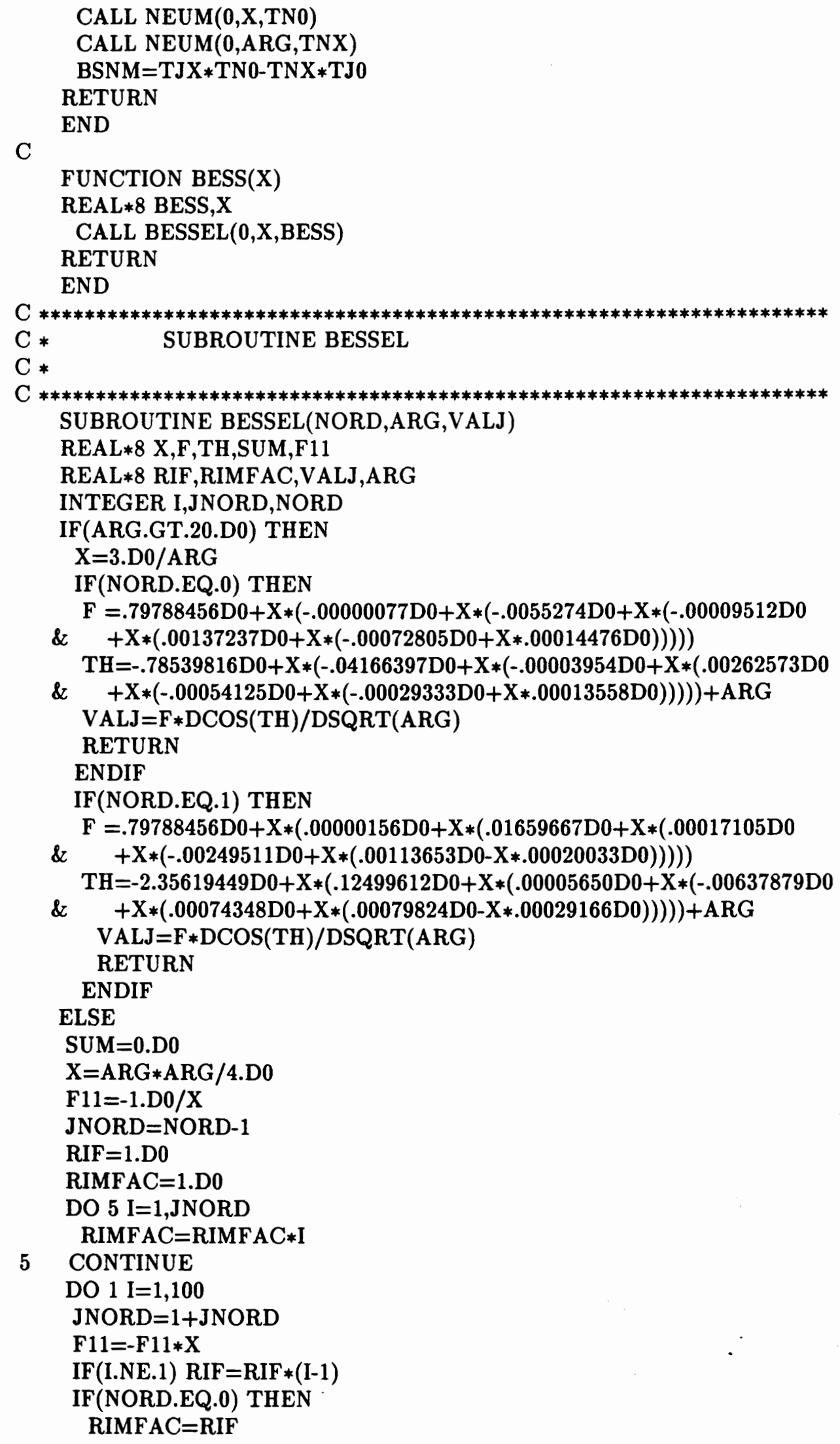




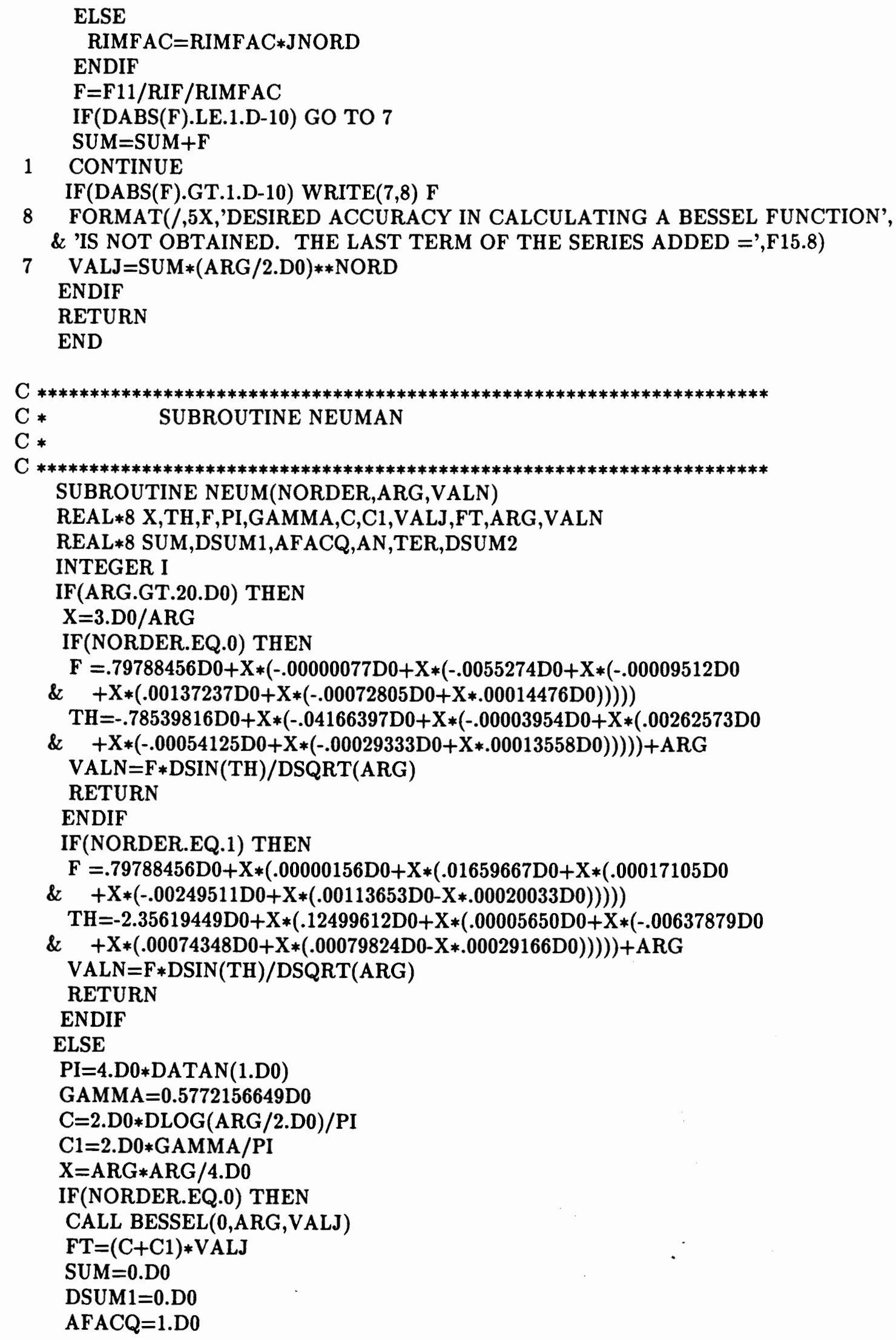




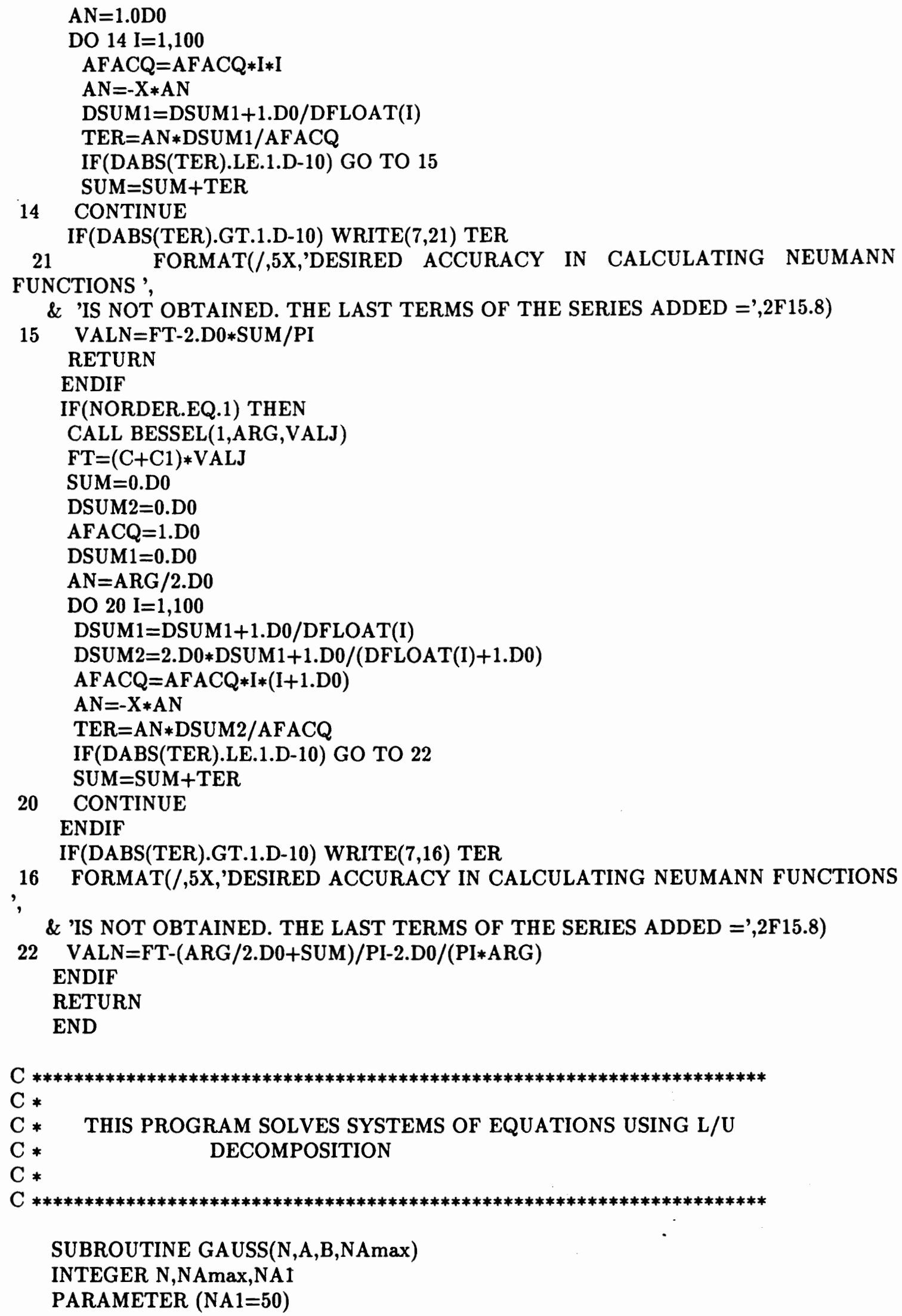


INTEGER IPIVOT(NA1)

REAL $* 8$ D

COMPLEX $* 16$ A(NAmax,NAmax),B(NAmax)

CALL LUDCMP(A,N,NAmax,IPIVOT,D)

CALL LUBKSB(A,N,NAmax,IPIVOT,B)

RETURN

END

SUBROUTINE LUDCMP(A,N,NP,INDX,D)

C*****FROM P.35 OF "NUMERICAL RECIPES"

C The input matrix is $\mathrm{A}(\mathrm{NP}, \mathrm{NP})$ with $\mathrm{NxN}$ elements used.

C On output $\mathrm{A}$ is the combined $\mathrm{L}-\mathrm{U}$ decomposition.

$\mathrm{C}$ The integer vector INDX(N) is used for ordering.

C Real D represents the sign of the determinant start.

C The determinant is found as D multiplied by all $A(I, I), I=1, N$.

$\mathrm{C} * * * * *$

INTEGER INDX(N),IMAX,N,NP,I,J,K,NMAX

REAL $* 8$ TINY

PARAMETER (NMAX $=100$, TINY $=1.0 \mathrm{E}-20$ )

REAL $* 8$ D,DUM,AAMAX,VV(NMAX)

C*****CHANGE NEXT LINE FROM REAL TO COMPLEX FOR COMPLEX APPLICATIONS

COMPLEX $* 16$ A(NP,NP),CDUM,SUM

$\mathrm{D}=1.0$

DO $12 \mathrm{I}=1, \mathrm{~N}$

AAMAX $=0$.

DO $11 \mathrm{~J}=1, \mathrm{~N}$

IF (ABS(A(I,J)).GT.AAMAX) AAMAX $=\operatorname{ABS}(\mathbf{A}(\mathbf{I}, \mathbf{J}))$

11 CONTINUE

IF (AAMAX.EQ.0.) THEN

PAUSE 'SINGULAR MATRIX.'

RETURN

ENDIF

$\mathrm{VV}(\mathrm{I})=1 . / \mathrm{AAMAX}$

12 CONTINUE

DO $19 \mathrm{~J}=1, \mathrm{~N}$

DO $14 \mathrm{I}=1, \mathrm{~J}-1$

SUM $=A(\mathrm{I}, \mathrm{J})$

DO $13 \mathrm{~K}=1, \mathrm{I}-1$

13 CONTINUE

$\mathrm{SUM}=\mathrm{SUM}-\mathrm{A}(\mathrm{I}, \mathrm{K}) * \mathrm{~A}(\mathrm{~K}, \mathrm{~J})$

$\mathrm{A}(\mathrm{I}, \mathrm{J})=\mathrm{SUM}$

14 CONTINUE

AAMAX $=0$.

DO $16 \mathrm{I}=\mathrm{J}, \mathrm{N}$

$\mathrm{SUM}=\mathrm{A}(\mathrm{I}, \mathrm{J})$

DO $15 \mathrm{~K}=1, \mathrm{~J}-1$

15 CONTINUE

SUM $=\mathrm{SUM}-\mathrm{A}(\mathrm{I}, \mathrm{K}) * \mathrm{~A}(\mathrm{~K}, \mathrm{~J})$

$A(I, J)=S U M$

$\mathrm{DUM}=\mathrm{VV}(\mathrm{I}) * \mathrm{ABS}(\mathrm{SUM})$

IF (DUM.GE.AAMAX) THEN

IMAX $=$ I 


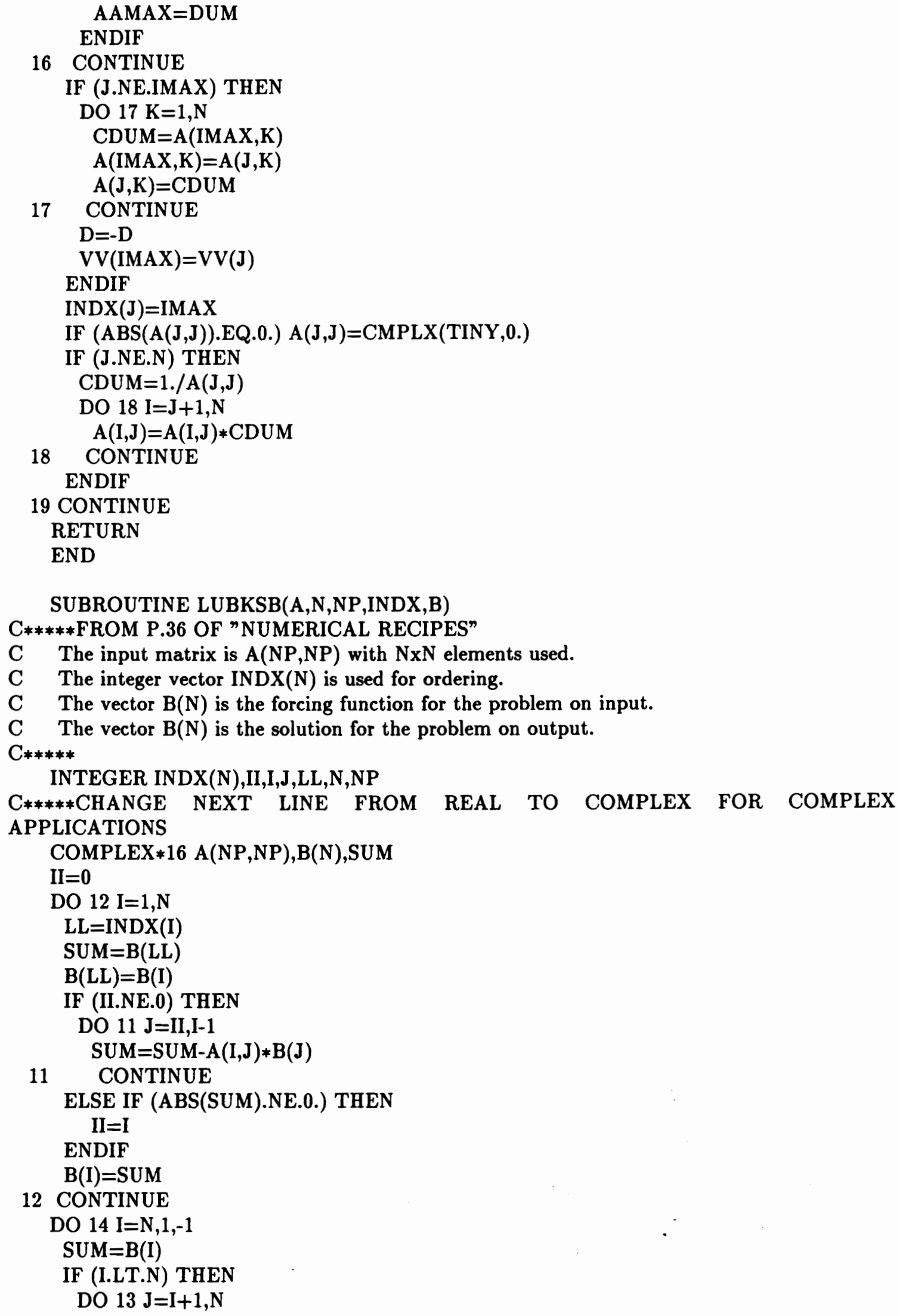


13 SUM=SUM-A

ENDIF

$\mathrm{B}(\mathrm{I})=\mathrm{SUM} / \mathrm{A}(\mathrm{I}, \mathrm{I})$

14 CONTINUE

RETURN

END 


\section{BIBLIOGRAPHY}

Balanis, C. B., (1989), "Advanced Engineering Electromagnetics", John Wiley \& Sons, N.Y.

Collin, R. E., (1960), "Field Theory of Guided Waves", Mc Grawhill, N.Y.

Felsen, L. B., and Marcuvitz, N., (1973), "Radiation and Scattering of Waves", Prentice Hall, N. J.

Harrington, R. F., (1961), "Time-Harmonic Electromagnetic Fields", McGraw-Hill Company, N.Y.

Harrington, R. F., (1968), "Field Computation by Moment Methods", Krieger, FA.

Lee, S. W., Jones, W. R., and Campbell, J. J., (1971), "Convergence of numerical solutions of Iris-type discontinuity problems" IEEE Trans. Microwave Theory Tech., vol. MTT-19, pp. 528-536, June.

Leroy, M., (1983), "On the convergence of numerical results in modal analysis" IEEE Trans. Antennas Propagation, vol. AP-31, No. 4, pp. 655-659, July.

Lighthill, M. J., (1958), "Introduction to Fourier Analysis and Generalised Functions", Cambridge Univ. Press, N. Y.

Marcuvitz, N., (1951), "Waveguide Handbook", McGraw-Hill, N.Y.

Mittra, R. and Lee, W. W. (1971), "Analytical techniques in the theory of guided waves", Macmillan, N.Y.

Mittra, R., Itoh, T., and Li, T. -s., (1972), "Analytical and numerical studies of the relative convergence phenomenon arising in the solution of an integral equation by the moment method", IEEE Trans. Microwave theory tech., vol. MTT-20, pp. 96-104, Feb. 
Mittra, R., (1963), "Relative Convergence of the Solution of a doubly Infinite Set of Equations", J. Res. Nat. Bur. Stand., vol. 67D, pp. 245-254, Mar. - Apr.

Morse, P. M., and Feshback, H., (1953), "Methods of theoretical Physics", McGraw-Hill, N.Y.

Riad, S. M., A. Elshabini-Riad, and M. A. Saed, (1989), "Wide-Band Measurement of the Complex Permittivity of Dielectric Materials Using a Wide-Band Cavity", IEEE Transactions on Instrumentation and Measurement, Volume 38, No.2, April.

Riad, S.M., Elshabini-Riad, A., Davis, W.A., Addington, S.J., Andrawis, M.Y., Bucca, S.E., Bunting, C.F., Ellis, E.R., Fidanboylu, K.M., Hayes, M.B. and $\mathrm{Su}$, W., (1990), "Wideband and Microwave Techniques for Material Characterization: Effect of Condcutor Properties and Interface on Dielectric Characterization, Final Report submitted to E. I. Du Pont, Electronics Department, Wilmington, Delaware, Sept.

Riad, S.M., Elshabini-Riad, A., Davis, W.A., Fidanboylu, K.M., Addington, S.J., Andrawis, M.Y., Bunting, C.F., Ellis, E.R., Hayes, M.B., Kunselman, G., and Su, W., (1991), "Development and Application of Microwave/TimeDomain Enviromental Chamber(s) for Material Characterization", Final Report submitted to E. I. Du Pont, Electronics Department, Wilmington, Delaware, Sept.

Riad, S.M., Davis, W.A., Elshabini-Riad, A., Fidanboylu, K.M.,Bucca, S., Su, W., Saed, M., Amey, D., Curilla, J., Lyles, J., Murphy, A., and Poulin, T. (1991), "Wideband and Microwave Material Characterization", $4^{\text {th }}$ ASM International Electronic Materials and Processing Conference. Canada, Aug.

Saed, M. A., (1987), "Dielectric Characterization Using a Wideband Dielectric Filled Cavity," Ph.D. dissertation in Electrical Engineering, Virginia Polytechnic Institute and State University, Blacksburg, Virginia. 
Saed, M. A., S. M. Riad, and W. A. Davis, (1990), "WideBand Dielectric Characterization Using a Dielectric Filled Cavity Adapted to the End of a Transmission Line", IEEE Transactions on Instrumentation and Measurement, Volume 39, No.3, June.

Shih, Y. C., and Gray, K., (1983), "Convergence of numerical solutions of step-type waveguide discontinuity problems by modal analysis", IEEE MTT-S Int. Microwave Symp. Dig., pp 233-235.

Stakgold, I., (1967), "Boundary Value Problems of athematical Physics", vol. I, Macmillan, N. Y.

Tai, C. T., (1972), "Dyadic Green's Functions in Electromagnetic Theory", Scranton, PA: Intext Educational Publishers.

Tyler, J. R., and Gasper, D. J., (1989), "Evaluation and Characterization of LowTemperature Cofired Ceramic Structures for Microwave Applications.", Proc. Int. Symp. on Microelect. pp390-399.

Van Bladel, J., (1964), "Electromagnetic Fields", McGrawhill, N.Y. 


\section{VITA}

Ms. Madeleine Younan Andrawis was born on November the $3^{r d}, 1953$, in Cairo, Egypt. She went to Catholic nuns school "Pensionnat De La Mere De Dieu" for all her precollege school years. She received her B.S. Degree in Electrical Engineering (Communications section), from Cairo University, Egypt, in 1977. She received her M.S. degree in Electrical Engineering from South Dakota State University, in 1983. She joined the Ph.D. graduate program in Electrical Engineering at Virginia Polytechnic Institute and State University in February 1988.

From 1978 to 1979, she has been working as planning engineer in the Arab States Broadcasting Union in the Sudan. From 1983 to 1987, she was teaching at South Dakota State University.

Madeleine enjoys social activities, music, traveling, and cake decorating. She is blessed with a loving and caring husband and three lovely children. Madeleine is a member of Eta Kappa Nu, and Phi Kappa Phi honor societies.

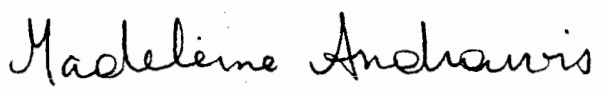

